\title{
Lattice Boltzmann models for the convection-diffusion equation: D2Q5 vs D2Q9
}

\author{
Like Li ${ }^{1}{ }^{*}$, Renwei Mei ${ }^{1}$, and James F. Klausner ${ }^{2}$
}

1. Department of Mechanical and Aerospace Engineering, University of Florida, Gainesville, FL 32611, USA

2. Department of Mechanical Engineering, Michigan State University, East Lansing, MI 48824, USA

* Corresponding author: $\underline{\text { likelichina@ufl.edu }}$

\begin{abstract}
A comparison study between the two most popular lattice Boltzmann (LB) models-D2Q5 and D2Q9 in two dimensions with five and nine discrete lattice velocities, respectively-for the convection-diffusion equation (CDE) for scalar transport is presented in this work. The local equilibria in both the discrete velocity space and the moment space are provided for the LB models examined, including the generalized D2Q5 model without high-order velocity terms and four various D2Q9 models frequently used in the literature. The boundary condition treatment is discussed with particular Dirichlet and Neumann boundary schemes presented for each LB model. In particular, the non-uniqueness in the splitting of the normal flux into three lattice directions in D2Q9 model for Neumann conditions is highlighted. Five representative numerical tests are presented to assess the performance of the D2Q5 and D2Q9 LB models. Performance metrics include the numerical accuracy and convergence orders of the interior distribution of the scalar variable $\phi$ and its derivatives as well as the boundary flux, boundary scalar values, and total boundary heat/mass transfer rate. The results demonstrate that (i) without the boundary effect, both the D2Q5 and D2Q9 models have second-order convergence for $\phi$ and its derivatives, and the D2Q5 model has smaller numerical errors at low and moderate Péclet numbers while the D2Q9 models have better accuracy at high Péclet numbers; (ii) for the CDE
\end{abstract}


with specific boundary conditions on straight walls, the D2Q5 model with the interpolation based boundary schemes is able to preserve the second-order accuracy for all the quantities of interest for both Dirichlet and Neumann problems; when the D2Q9 models are applied, these boundary schemes result in second- and first-order accurate $\phi$ field for the Dirichlet and Neumann problems, respectively; in addition, the convergence orders for the interior derivatives and the boundary flux or boundary values are also degraded for both types of problems; (iii) when curved geometry is encountered, the D2Q5 model also shows better accuracy and/or higher convergence orders than the D2Q9 models. Clearly, D2Q5 is more appealing to D2Q9 for the $\mathrm{CDE}$ when the convection is not very strong and the boundary effect is significant. The same behavior in the respective 3-D numerical solutions is observed when the performance of D3Q7 and D3Q19 LB models are compared.

Keywords: lattice Boltzmann (LB); convection-diffusion equation; D2Q5; D2Q9; Dirichlet boundary condition; Neumann boundary condition.

\section{Introduction}

The lattice Boltzmann (LB) method simulates the particle interactions on a discrete phase space with a specific set of discrete velocities. The evolution of the microscopic particle distribution functions (also called density distribution functions) is governed by the lattice Boltzmann equation (LBE) [1-3], from which the macroscopic Navier-Stokes equations (NSE) can be recovered via a multiscale analysis such as the Chapman-Enskog expansion [4-6] or the asymptotic analysis [7]. The typical two- and three-dimensional (2D, 3D) lattices include D2Q9, D3Q15 and D3Q19, respectively, (D $n$ Q $m$ denotes $m$ discrete velocities in $n$ dimensions) in the LB models for fluid dynamics simulations $[6,8,9]$. 
Extension of the hydrodynamic version of the LB models to simulate heat and mass transfer problems has been conducted since the beginning of the development of the LB method [10-26]. The technique of treating the thermal and mass transport processes as general convection and diffusion of a scalar variable (temperature/concentration in heat/mass transfer) has achieved much success. A variety of LB models have been developed to recover the general convection-diffusion equation (CDE) [15, 17-26]. In these scalar transport LB models, a separate set of microscopic distribution functions is defined, and the macroscopic temperature or concentration is obtained from the zeroth moment of these distribution functions. This proves to be much more effective and efficient than constructing specific LB models by treating the macroscopic internal or total energy as a higher-order moment of the density distribution functions as in $[14,16]$.

There exist two most popular types of lattice model, i.e., D2Q9 and D2Q5 in 2D, and D3Q15/D3Q19 and D3Q7 in 3D, for solving the CDE. The D2Q9 and D3Q15/D3Q19 LB models $[13,14,16,18,21-23,26]$ involve both orthogonal and diagonal lattice velocity vectors and they are direct extensions from the hydrodynamic versions. The D2Q5 and D3Q7 models $[17,19,20-22,24,25]$ have only orthogonal lattice vectors. They are simpler in form and easier to code for curved boundary. While it is obvious that the D2Q5 model is more computationally efficient than D2Q9 (this is more pronounced in 3D cases), one would tend to believe that D2Q9 could be more accurate and applicable to more complex situations compared to D2Q5 because it has more lattice vectors and components of the distribution function.

Another crucial aspect in assessing LB models is to ascertain how the boundary condition treatment affects the performance of the respective model. With the discrete lattice velocities only in the orthogonal directions, D2Q5 requires a much simpler boundary treatment 
compared to D2Q9. In particular, for D2Q5, second-order accurate boundary schemes for both Đirichlet and Neumann conditions have been proposed in [24] with a detailed asymptotic analysis and numerical verification. Their application to curved boundaries was also examined in [24]. For D2Q9, although various boundary schemes have been proposed in the literature, there still exists ambiguity regarding their orders of accuracy, especially for Neumann conditions and for both types of boundary conditions on curved boundaries.

In this paper, we try to address which of the above mentioned two types of LB models is preferred by presenting direct comparisons of the numerical solutions for scalar transport obtained using these two models for the CDE. The main focus is on 2D cases, i.e., D2Q5 vs D2Q9; and similar conclusions in 3D are verified by comparing results from using D3Q7 and D3Q19 models. The effects of the boundary condition treatment (for both Dirichlet and Neumann conditions) on the accuracy and convergence of the LB models are also examined in detail.

A series of numerical tests, including isotropic/anisotropic convection-diffusion with straight/curved boundaries, are presented to compare the performance of D2Q5/D3Q7 and D2Q9/D3Q19 models. The numerically examined quantities include the interior distribution of the scalar variable $\phi$ and its derivatives in both Dirichlet and Neumann problems, the local boundary flux and overall boundary heat/mass flow rate over the entire boundary in Dirichlet problems, and the boundary scalar values in Neumann problems. From both model analysis and numerical testing it becomes evident that the D2Q5/D3Q7 models are more attractive to the D2Q9/D3Q19 models available in the literature. Thus the D2Q5/D3Q7 models for the CDE are recommended in general. It should be noted, however, opportunities exist for the construction of optimized D2Q9 models with improved boundary condition treatments, which could have better 
numerical accuracy than the basic D2Q5 model, considering the flexibility in the model construction with additional lattice vectors in D2Q9. This is out of the scope of the present paper which focuses on the comparison of the existing D2Q5 and D2Q9 models.

\section{LB models for the convection-diffusion equation}

The macroscopic convection-diffusion equation (CDE) can be written as

$$
\frac{\partial \phi}{\partial t}+\frac{\partial}{\partial x_{j}}\left(v_{j} \phi\right)=\frac{\partial}{\partial x_{i}}\left(D_{i j} \frac{\partial \phi}{\partial x_{j}}\right)+G
$$

where $\phi$ is a scalar variable such as temperature in heat transfer or concentration in mass transfer problems, $t$ is the time, $v_{j}$ is the $j$ th-component of the velocity vector $\mathbf{u}$ in the $x_{j}$-direction, $D_{i j}$ is the diffusion coefficient, and $G$ is the general source term.

A microscopic distribution function, $g(\mathbf{x}, \xi, t)$, is defined in the discrete velocity space to recover the CDE (1) and its evolution is governed by the lattice Boltzmann equation (LBE)

$$
g_{\alpha}\left(\mathbf{x}+\mathbf{e}_{\alpha} \delta t, t+\delta t\right)-g_{\alpha}(\mathbf{x}, t)=\left[\mathbf{L} \cdot\left(\mathbf{g}-\mathbf{g}^{\mathrm{eq}}\right)(\mathbf{x}, t)\right]_{\alpha}+\omega_{\alpha} G(\mathbf{x}, t) \delta t
$$

where $g_{\alpha}(\mathbf{x}, t) \equiv g\left(\mathbf{x}, \xi_{\alpha}, t\right), \xi$ is the particle velocity vector and it is discretized to a small set of discrete velocities $\left\{\boldsymbol{\xi}_{\alpha} \mid \alpha=0,1, \ldots, m-1\right\}, \mathbf{e}_{\alpha}$ is the $\alpha$ th discrete velocity vector, $\delta t$ is the time step, $\mathbf{L}$ is the collision operator in the LBE method, $g_{\alpha}^{\text {eq }}(\mathbf{x}, t)$ is the equilibrium distribution function, and $\omega_{\alpha}$ is the weight coefficient. The macroscopic scalar variable can be obtained from

$$
\phi(\mathbf{x}, t)=\sum_{\alpha=0}^{m-1} g_{\alpha}(\mathbf{x}, t)
$$

For unit lattice spacing $\delta x=1$ and unit time step $\delta t=1$ in 2D problems, the sets of $\left\{\mathbf{e}_{\alpha}\right\}$ for the two most widely used lattice structures, D2Q5 and D2Q9, are defined as 


$$
\begin{aligned}
& \mathbf{e}_{\alpha}=\left\{\begin{array}{ll}
(0,0), & \alpha=0 \\
( \pm 1,0),(0, \pm 1), \alpha=1,2,3,4
\end{array},\right. \text { for D2Q5, and } \\
& \mathbf{e}_{\alpha}= \begin{cases}(0,0), & \alpha=0 \\
(\cos [(\alpha-1) \pi / 2], \sin [(\alpha-1) \pi / 2]), & \alpha=1,2,3,4, \text { for D2Q9. } \\
\sqrt{2}(\cos [(2 \alpha-1) \pi / 4], \sin [(2 \alpha-1) \pi / 4]), & \alpha=5,6,7,8\end{cases}
\end{aligned}
$$

The equilibrium distribution function can be similarly defined as that in the hydrodynamic LB model by replacing the density $\rho$ with the scalar variable $\phi$

$$
g_{\alpha}^{\mathrm{eq}}=\omega_{\alpha} \phi\left[1+\frac{\mathbf{e}_{\alpha} \cdot \mathbf{u}}{c_{s}^{2}}+\frac{\left(\mathbf{e}_{\alpha} \cdot \mathbf{u}\right)^{2}}{2 c_{s}^{4}}-\frac{\mathbf{u}^{2}}{2 c_{s}^{2}}\right],
$$

where the arguments $(\mathbf{x}, t)$ are dropped for brevity (all the distribution functions $g_{\alpha}$ and $g_{\alpha}^{\text {eq }}$ below are obtained at $(\mathbf{x}, t)$ if not given explicitly) and $c_{s}$ is the speed of sound with $c_{s}=c / \sqrt{3}=(\delta x / \delta t) / \sqrt{3}=1 / \sqrt{3}$. Realizing that only the zeroth-order moment of the distribution functions needs to be conserved (see Eq. (3)) to recover the CDE, one can drop the higher order velocity terms in Eq. (5a) and simplify it to be $[17,20]$

$$
g_{\alpha}^{\mathrm{eq}}=\omega_{\alpha} \phi\left(1+\frac{\mathbf{e}_{\alpha} \cdot \mathbf{u}}{c_{s}^{2}}\right)
$$

It should be noted that the choice of the coefficients for each term in Eqs. (5a, 5b) is not unique. For general cases, they can be rewritten as

$$
g_{\alpha}^{\mathrm{eq}}=\omega_{\alpha} \phi\left[1+a_{\alpha} \frac{\mathbf{e}_{\alpha} \cdot \mathbf{u}}{c_{s}^{2}}+b_{\alpha} \frac{\left(\mathbf{e}_{\alpha} \cdot \mathbf{u}\right)^{2}}{2 c_{s}^{4}}-c_{\alpha} \frac{\mathbf{u}^{2}}{2 c_{s}^{2}}\right],
$$

where $a_{\alpha}, b_{\alpha}$, and $c_{\alpha}$ are constants. The numerical values of the constants and the weight coefficient $\omega_{\alpha}$ will be given according to the specific models discussed below.

Regarding the collision operator $\mathbf{L}$, there are three popular models in the literature. The earliest and simplest one is the BGK [27] or single-relaxation-time (SRT) model 


$$
\left[\mathbf{L} \cdot\left(\mathbf{g}-\mathbf{g}^{\mathrm{eq}}\right)\right]_{\alpha}=\lambda\left(g_{\alpha}-g_{\alpha}^{\mathrm{eq}}\right)=-\frac{1}{\tau}\left(g_{\alpha}-g_{\alpha}^{\mathrm{eq}}\right),
$$

where $\lambda$ is the eigenvalue and $\tau=-1 / \lambda$ is usually called the relaxation coefficient/parameter.

The second one is the two-relaxation-time (TRT) model introduced by Ginzburg and Ginzburg et al. [21, 28], in which the distribution function is decomposed into its symmetric (even) and antisymmetric (odd) parts: $g_{\alpha}=g_{\alpha}^{+}+g_{\alpha}^{-}$, with $g_{\alpha}^{+}=g_{\bar{\alpha}}^{+}, g_{\alpha}^{-}=-g_{\bar{\alpha}}^{-}$and $\mathbf{e}_{\bar{\alpha}}=-\mathbf{e}_{\alpha}$. This decomposition also applies to its equilibrium: $g_{\alpha}^{\mathrm{eq}}=g_{\alpha}^{\mathrm{eq},+}+g_{\alpha}^{\mathrm{eq},-}$. A pair of even and odd relation coefficients is thus used and the collision operator becomes

$$
\left[\mathbf{L} \cdot\left(\mathbf{g}-\mathbf{g}^{\mathrm{eq}}\right)\right]_{\alpha}=\lambda_{\alpha}^{+}\left(g_{\alpha}^{+}-g_{\alpha}^{\mathrm{eq},+}\right)+\lambda_{\alpha}^{-}\left(g_{\alpha}^{-}-g_{\alpha}^{\mathrm{eq},-}\right) .
$$

In the most general multiple-relaxation-time (MRT) model, a transformation matrix $\mathbf{M}$ is introduced to map the distribution functions to the moment space: $\mathbf{m}=\mathbf{M} \cdot \mathbf{g}$ and $\mathbf{m}^{\mathrm{eq}}=\mathbf{M} \cdot \mathbf{g}^{\mathrm{eq}}$, and the collision operator is

$$
\left[\mathbf{L} \cdot\left(\mathbf{g}-\mathbf{g}^{\mathrm{eq}}\right)\right]_{\alpha}=-\left[\mathbf{M}^{-1} \mathbf{S M} \cdot\left(\mathbf{g}-\mathbf{g}^{\mathrm{eq}}\right)\right]_{\alpha}=\left[\mathbf{M}^{-1} \mathbf{S} \cdot\left(\mathbf{m}-\mathbf{m}^{\mathrm{eq}}\right)\right]_{\alpha},
$$

where $\mathbf{S}$ is a matrix of relaxation time coefficients. The transformation matrices for D2Q5 and D2Q9 LB models are [19, 20, 24-26, 29, 30]

$$
\mathbf{M}=\left[\begin{array}{rrrrr}
1 & 1 & 1 & 1 & 1 \\
0 & 1 & -1 & 0 & 0 \\
0 & 0 & 0 & 1 & -1 \\
4 & -1 & -1 & -1 & -1 \\
0 & 1 & 1 & -1 & -1
\end{array}\right], \mathbf{S}^{-1}=\left[\begin{array}{ccccc}
\tau_{0} & 0 & 0 & 0 & 0 \\
0 & \tau_{x x} & \tau_{x y} & 0 & 0 \\
0 & \tau_{y x} & \tau_{y y} & 0 & 0 \\
0 & 0 & 0 & \tau_{3} & 0 \\
0 & 0 & 0 & 0 & \tau_{4}
\end{array}\right] \text {, for D2Q5, and }
$$




$$
\mathbf{M}=\left[\begin{array}{ccccccccc}
1 & 1 & 1 & 1 & 1 & 1 & 1 & 1 & 1 \\
-4 & -1 & -1 & -1 & -1 & 2 & 2 & 2 & 2 \\
4 & -2 & -2 & -2 & -2 & 1 & 1 & 1 & 1 \\
0 & 1 & 0 & -1 & 0 & 1 & -1 & -1 & 1 \\
0 & -2 & 0 & 2 & 0 & 1 & -1 & -1 & 1 \\
0 & 0 & 1 & 0 & -1 & 1 & 1 & -1 & -1 \\
0 & 0 & -2 & 0 & 2 & 1 & 1 & -1 & -1 \\
0 & 1 & -1 & 1 & -1 & 0 & 0 & 0 & 0 \\
0 & 0 & 0 & 0 & 0 & 1 & -1 & 1 & -1
\end{array}\right], \mathbf{S}^{-1}=\left[\begin{array}{ccccccccc}
\tau_{0} & 0 & 0 & 0 & 0 & 0 & 0 & 0 & 0 \\
0 & \tau_{1} & 0 & 0 & 0 & 0 & 0 & 0 & 0 \\
0 & 0 & \tau_{2} & 0 & 0 & 0 & 0 & 0 & 0 \\
0 & 0 & 0 & \tau_{x x} & 0 & \tau_{x y} & 0 & 0 & 0 \\
0 & 0 & 0 & 0 & \tau_{4} & 0 & 0 & 0 & 0 \\
0 & 0 & 0 & \tau_{y x} & 0 & \tau_{y y} & 0 & 0 & 0 \\
0 & 0 & 0 & 0 & 0 & 0 & \tau_{6} & 0 & 0 \\
0 & 0 & 0 & 0 & 0 & 0 & 0 & \tau_{7} & 0 \\
0 & 0 & 0 & 0 & 0 & 0 & 0 & 0 & \tau_{8}
\end{array}\right],
$$

where the relaxation coefficients need to satisfy

$$
\tau_{i j}=\frac{1}{2} \delta_{i j}+\frac{\delta t}{\varepsilon_{D} \delta x^{2}} D_{i j},
$$

in order to recover the anisotropic $\mathrm{CDE}$ (1). The constant $\varepsilon_{D}$ in Eq. (8) is $\varepsilon_{D}=(4-a) / 10$ (see Table 1 for the definition of $a$, and $\varepsilon_{D}=1 / 3$ at $a=2 / 3$ ) for D2Q5 [24, 25, 31, 32] and $\varepsilon_{D}=1 / 3$ for D2Q9 [26]. It should be noted that the MRT collision operator in Eq. (6c) reduces to the BGK version in Eq. (6a) when all the diagonal elements in the $\mathbf{S}^{-1}$ matrix are equal to $\tau$, and the offdiagonal ones are set to zeros; and it reduces to the TRT version in Eq. (6b) when its even/odd eigenvalues take specific values, $\lambda_{\alpha}^{+} / \lambda_{\alpha}^{-}$, respectively.

It is noted that one can also tune the relaxation coefficients in the $\mathbf{S}$ matrix to construct other specific LB models. For instance, Huang and Wu [26] modified $\mathbf{S}$ in their D2Q9 MRT model to eliminate some "deviation" terms that exist in convection-diffusion problems with strong convection and a high Mach number. Their matrix $\mathbf{S}$ can be rewritten as 


$$
\mathbf{S}^{-1}=\left[\begin{array}{ccccccccc}
\tau_{0} & 0 & 0 & 0 & 0 & 0 & 0 & 0 & 0 \\
0 & \tau_{1} & 0 & 0 & 0 & 0 & 0 & 0 & 0 \\
0 & 0 & \tau_{2} & 0 & 0 & 0 & 0 & 0 & 0 \\
0 & 0 & 0 & \tau_{x x} & \tau_{34} & \tau_{x y} & \tau_{36} & 0 & 0 \\
0 & 0 & 0 & 0 & \tau_{4} & 0 & 0 & 0 & 0 \\
0 & 0 & 0 & \tau_{y x} & \tau_{54} & \tau_{y y} & \tau_{56} & 0 & 0 \\
0 & 0 & 0 & 0 & 0 & 0 & \tau_{6} & 0 & 0 \\
0 & 0 & 0 & 0 & 0 & 0 & 0 & \tau_{7} & 0 \\
0 & 0 & 0 & 0 & 0 & 0 & 0 & 0 & \tau_{8}
\end{array}\right] \text {, for D2Q9, }
$$

with $\tau_{34}=3 D_{x x}, \tau_{36}=3 D_{x y}, \tau_{54}=3 D_{y x}$, and $\tau_{56}=3 D_{y y}$ in addition to those given in Eq. (8).

For general purposes, we formulate the LB models used in this work in the MRT framework and the LBE evolution equation (2) is solved in two steps

collision step:

$$
\hat{g}_{\alpha}(\mathbf{x}, t)=g_{\alpha}(\mathbf{x}, t)-\left[\mathbf{M}^{-1} \mathbf{S} \cdot\left(\mathbf{m}-\mathbf{m}^{\mathrm{eq}}\right)(\mathbf{x}, t)\right]_{\alpha}+\omega_{\alpha} G(\mathbf{x}, t) \delta t \text {, and }
$$

streaming step:

$$
g_{\alpha}\left(\mathbf{x}+\mathbf{e}_{\alpha} \delta t, t+\delta t\right)=\hat{g}_{\alpha}(\mathbf{x}, t)
$$

where $\hat{g}_{\alpha}$ represents the post-collision state.

One of the main objectives of this study is to show a direct comparison between the D2Q5 and D2Q9 models for representative convection-diffusion problems, and we summarize the expressions for $\mathbf{g}^{\mathrm{eq}}$ and $\mathbf{m}^{\mathrm{eq}}$ for each LB model studied in this work in Table 1. The information for the D3Q7 and D3Q19 LB models is provided in the Appendix. 
Table 1. The equilibria $\mathrm{g}^{\mathrm{eq}}, \mathrm{m}^{\mathrm{eq}}$ and the weight coefficient $\omega_{\alpha}$ in the various $2 \mathrm{D}$ LB models.

\begin{tabular}{|c|c|c|c|}
\hline $\begin{array}{l}\text { MRT-LB } \\
\text { models }\end{array}$ & $\begin{array}{l}g_{\alpha}^{\mathrm{eq}}=\omega_{\alpha} \phi\left[1+a_{\alpha} \frac{\mathbf{e}_{\alpha} \cdot \mathbf{u}}{c_{s}^{2}}\right. \\
\left.+b_{\alpha} \frac{\left(\mathbf{e}_{\alpha} \cdot \mathbf{u}\right)^{2}}{2 c_{s}^{4}}-c_{\alpha} \frac{\mathbf{u}^{2}}{2 c_{s}^{2}}\right]\end{array}$ & $\mathbf{m}^{\mathrm{eq}}=\mathbf{M} \cdot \mathbf{g}^{\mathrm{eq}}$ & $\omega_{\alpha}$ \\
\hline $\begin{array}{c}\text { D2Q5 } \\
(\mathbf{S} \text { in Eq. (7a) })\end{array}$ & $\begin{aligned} a_{\alpha} & =\frac{10}{3(4-a)} \\
b_{\alpha} & =0, c_{\alpha}=0\end{aligned}$ & $\phi\left(1, u_{x}, u_{y}, a, 0,0\right)^{\mathrm{T}}$ & $\left\{\begin{array}{l}\frac{1+a}{5}, \alpha=0 \\
\frac{4-a}{20}, \alpha=1-4\end{array}\right.$ \\
\hline $\begin{array}{c}\text { D2Q9-I } \\
(\mathbf{S} \text { in Eq. (7b)) }\end{array}$ & $a_{\alpha}=1, b_{\alpha}=0, c_{\alpha}=0$ & $\phi\left(1,-2,1, u_{x},-u_{x}, u_{y},-u_{y}, 0,0\right)^{\mathrm{T}}$ & $\begin{cases}4 / 9, & \alpha=0 \\
1 / 9, & \alpha=1-4 \\
1 / 36, & \alpha=5-8\end{cases}$ \\
\hline $\begin{array}{c}\text { D2Q9-II } \\
(\mathbf{S} \text { in Eq. (7b)) }\end{array}$ & $a_{\alpha}=1, b_{\alpha}=1, c_{\alpha}=1$ & $\phi\left(1,-2+3 \mathbf{u}^{2}, 1-3 \mathbf{u}^{2}, u_{x},-u_{x}, u_{y},-u_{y}, u_{x}^{2}-u_{y}^{2}, u_{x} u_{y}\right)^{\mathrm{T}}$ & $\left\{\begin{array}{l}4 / 9, \quad \alpha=0 \\
1 / 9, \quad \alpha=1-4 \\
1 / 36, \alpha=5-8\end{array}\right.$ \\
\hline $\begin{array}{c}\text { D2Q9-III } \\
(\mathbf{S} \text { in Eq. (9)) }\end{array}$ & $\begin{array}{c}a_{\alpha}= \begin{cases}0, & \alpha=0 \\
2, & \alpha=1-4, \\
1 / 2, & \alpha=5-8\end{cases} \\
b_{\alpha}=0, c_{\alpha}=0\end{array}$ & $\phi\left(1,-2,2, u_{x},-u_{x}, u_{y},-u_{y}, 0,0\right)^{\mathrm{T}}$ & $\left\{\begin{array}{l}5 / 9, \alpha=0 \\
1 / 18, \alpha=1-4 \\
1 / 18, \alpha=5-8\end{array}\right.$ \\
\hline $\begin{array}{c}\text { D2Q9-IV } \\
\text { (S in Eq. (7b)) }\end{array}$ & $\begin{array}{l}a_{\alpha}= \begin{cases}0, & \alpha=0 \\
2, & \alpha=1-4, \\
1 / 2, & \alpha=5-8\end{cases} \\
b_{\alpha}= \begin{cases}0, & \alpha=0 \\
2, & \alpha=1-4, \\
1 / 2, & \alpha=5-8\end{cases} \\
c_{\alpha}= \begin{cases}4 / 5, \alpha=0 \\
2, & \alpha=1-4 \\
1 / 2, & \alpha=5-8\end{cases} \end{array}$ & $\phi\left(1,-2+3 \mathbf{u}^{2}, 2-3 \mathbf{u}^{2}, u_{x},-u_{x}, u_{y},-u_{y}, u_{x}^{2}-u_{y}^{2}, u_{x} u_{y}\right)^{\mathrm{T}}$ & $\begin{array}{l}5 / 9, \alpha=0 \\
1 / 18, \alpha=1-4 \\
1 / 18, \alpha=5-8\end{array}$ \\
\hline
\end{tabular}

Remark I: The constant $a$ in the D2Q5 model in Table 1 is bounded by $a \in(-1,4)$. In [20, 24, 32-37], $a=2 / 3$ was used which gives $g_{\alpha}^{\mathrm{eq}}=\omega_{\alpha} \phi\left(1+\frac{\mathbf{e}_{\alpha} \cdot \mathbf{u}}{c_{s}^{2}}\right)$ and $\omega_{0}=2 \omega_{1,2,3,4}=1 / 3$. This is the form of the D2Q5 model studied in the present work if not otherwise stated. Notably, the 
effect of $a$ on the accuracy and stability of the LBE solutions was studied in [25, 31]. It should be noted that the fourth row of the $\mathbf{M}$ matrix in $[25,31]$ has an opposite sign as that in Eq. (7a), and thus the constant $a$ is also of opposite sign as that in Table 1 .

Remark II: D2Q9-II in Table 1 is formulated the same way as that for the hydrodynamic version for fluid flow. D2Q9-I can be considered as a simplified version of D2Q9-II without the higher order velocity terms in $\mathbf{g}^{\mathrm{eq}}$ and $\mathbf{m}^{\mathrm{eq}}$. D2Q9-I and D2Q9-II are the most popular LB models studied in the literature for the 9-velocity lattice structure. D2Q9-III is the modified MRT LB model by Huang and Wu [26] for which the relaxation matrix is given in Eq. (9), noting that the original matrix $\mathbf{S}$ instead of $\mathbf{S}^{-1}$ is given in [26]. D2Q9-IV has the same "mass" and convection weights as D2Q9-III, and it includes the higher order velocity terms. In [26], the models D2Q9II, III, IV were denoted as MRT-B1, -A, -B2, respectively. Another MRT-B3 model, which is the same as D2Q9-III but with $\mathbf{S}$ in Eq. (7b) rather than Eq. (9), was also studied in [26].

Remark III: The relaxation coefficients $\tau_{x x}, \tau_{x y}, \tau_{y x}$, and $\tau_{y y}$, are determined by the diffusion coefficient $D_{i j}$ as shown in Eq. (8). The other relaxation coefficients are free with a lower bound $\tau_{P}>0.5\left(P=0,3,4\right.$ in D2Q5 and 0, 1, 2, 4, 6, 7, 8 in D2Q9). Importantly, the expressions for $\mathbf{m}^{\mathrm{eq}}$ in Table 1 clearly show that $\tau_{4}$ and $\tau_{6}$ in the D2Q9 models are also related to the respective convection terms $u_{x} \phi$ and $-u_{y} \phi$. According to the TRT LB model [21, 28], these are also antisymmetric (odd) components, thus $\tau_{4}=\tau_{x x}$ and $\tau_{6}=\tau_{y y}$ are used throughout this work. In addition, $\tau_{0}$ is the relaxation coefficient for the conserved scalar variable $\phi$, which is the first element in $\mathbf{m}^{\mathrm{eq}}$ for each of the LB models in Table 1 . In the hydrodynamic LB models, the relaxation coefficients for the conserved moments of mass and momentum are set to zero [29]. As an analogy, one can set $\tau_{0}=0$, for which the choice of $m_{\alpha=0}^{\text {eq }}$ does not come into play, thus $m_{\alpha=0}^{\text {eq }}=0$ was used in [19, 24, 32-34, 36, 37]. Notably, d'Humieres and Ginzburg [38] derived that in the 
TRT model, the combination of the two relaxation coefficient, $\Lambda=\left(\tau_{D}-\frac{1}{2}\right)\left(\tau_{P}-\frac{1}{2}\right)$, "magically" determines the LB solution for the CDE. For consistency, we use $\tau_{P}=1$ (including $\tau_{0}=1$ ) for all the simulation results shown in this paper.

\section{Boundary conditions in the LB models}

Since the LB method deals with the distribution functions, the conversion of a macroscopic boundary condition into that for the microscopic distribution functions is of essential significance in LB modeling. The most widely encountered boundary conditions for the CDE include the Dirichlet (boundary value) condition, Neumann (flux) condition and their combination, the mixed (Robin) condition. They can be written in a general form as

$$
\sigma_{1} \frac{\partial \phi}{\partial n}+\sigma_{2} \phi=\sigma_{3}
$$

where $n$ is the inward normal of the local boundary geometry, $\sigma_{1}, \sigma_{2}$, and $\sigma_{3}$ are given functions on the boundary. Eq. (11) reduces to the Dirichlet condition $\phi_{w}=\Phi_{d}$ when $\sigma_{1}=0$, and to the Neumann condition $-D_{n} \frac{\partial \phi}{\partial n}=\Phi_{n}$ when $\sigma_{2}=0$.

The lattice cut by the boundary wall with arbitrary intersection distance is illustrated in Fig. 1. The lattice spacing is set to be unity $(\delta x=1)$, and the fraction of the intersected link in the field to be solved is denoted by $\Delta=\left\|\mathbf{x}_{f}-\mathbf{x}_{w}\right\| /\left\|\mathbf{x}_{f}-\mathbf{x}_{e}\right\|$ with $0 \leq \Delta \leq 1$. A boundary condition treatment is required during the streaming step (Eq. (10b)) when unknown populations from the exterior nodes ( $\mathbf{x}_{e}$ in Fig. 1) to the "field" nodes $\left(\mathbf{x}_{f}\right)$ are encountered. 


\subsection{Halfway boundary schemes at $\Delta=0.5$}

The halfway intersection $(\Delta=0.5)$ represents the simplest case. For the Dirichlet boundary condition $\phi_{w}=\Phi_{d}$, the most popular "anti-bounce-back" scheme can be written as [39] $g_{\bar{\alpha}}\left(\mathbf{x}_{f}, t+\delta t\right)=-\hat{g}_{\alpha}\left(\mathbf{x}_{f}, t\right)+2 g_{\alpha}^{\mathrm{eq},+}\left(\mathbf{x}_{w}, t+\delta t\right)=-\hat{g}_{\alpha}\left(\mathbf{x}_{f}, t\right)+2 \omega_{\alpha} \Phi_{d}\left[1+b_{\alpha} \frac{\left(\mathbf{e}_{\alpha} \cdot \mathbf{u}\right)^{2}}{2 c_{s}^{4}}-c_{\alpha} \frac{\mathbf{u}^{2}}{2 c_{s}^{2}}\right]$.

The high order velocity terms are usually neglected [20, 24-26], and the anti-bounce-back Dirichlet schemes for the D2Q5 and D2Q9 models in Table 1 become

$$
\begin{aligned}
& \text { D2Q5: } g_{\bar{\alpha}}\left(\mathbf{x}_{f}, t+\delta t\right)=-\hat{g}_{\alpha}\left(\mathbf{x}_{f}, t\right)+\frac{4-a}{10} \Phi_{d}, \alpha=1-4, \\
& \text { D2Q9-I, D2Q9-II: } g_{\bar{\alpha}}\left(\mathbf{x}_{f}, t+\delta t\right)=\left\{\begin{array}{l}
-\hat{g}_{\alpha}\left(\mathbf{x}_{f}, t\right)+\frac{2}{9} \Phi_{d}, \alpha=1-4, \\
-\hat{g}_{\alpha}\left(\mathbf{x}_{f}, t\right)+\frac{1}{18} \Phi_{d}, \alpha=5-8,
\end{array}\right. \\
& \text { D2Q9-III, D2Q9-IV: } g_{\bar{\alpha}}\left(\mathbf{x}_{f}, t+\delta t\right)=-\hat{g}_{\alpha}\left(\mathbf{x}_{f}, t\right)+\frac{1}{9} \Phi_{d}, \alpha=1-8 .
\end{aligned}
$$

For the zero-flux Neumann boundary condition $\Phi_{n}=0$, the "bounce-back" scheme, $g_{\bar{\alpha}}\left(\mathbf{x}_{f}, t+\delta t\right)=\hat{g}_{\alpha}\left(\mathbf{x}_{f}, t\right)$, which is widely used in the hydrodynamic LB models to impose a no-slip condition for momentum can be similarly implemented $[19,25]$. For the general non-zero Neumann condition $-D_{n} \frac{\partial \phi}{\partial n}=\Phi_{n}$, Yoshida and Nagaoka [20] proposed a convenient scheme for the D2Q5 model. Using the notations in this work, it can be written as

$$
\text { D2Q5: } g_{\bar{\alpha}}\left(\mathbf{x}_{f}, t+\delta t\right)=\hat{g}_{\alpha}\left(\mathbf{x}_{f}, t\right)+\Phi_{n \bar{\alpha}}, \alpha=1-4,
$$

where $\Phi_{n \bar{\alpha}}$ is the boundary flux along the $\mathbf{e}_{\bar{\alpha}}$ direction from the exterior to the field. When the normal $\mathbf{n}$ is aligned with $\mathbf{e}_{\bar{\alpha}}, \Phi_{n \bar{\alpha}}=\Phi_{n}=-D_{n} \frac{\partial \phi}{\partial n}$ can be directly used. 
The Neumann boundary condition for the D2Q9 model is more complex than that for D2Q5 as one needs to split the boundary flux into those along three different lattice velocity directions. As shown in Fig. 1b, the weighted Neumann boundary scheme can be constructed as

$$
\text { D2Q9: }\left\{\begin{array}{l}
g_{1}\left(\mathbf{x}_{f}, t+\delta t\right)=\hat{g}_{3}\left(\mathbf{x}_{f}, t\right)+\kappa_{r} \Phi_{n}, \\
g_{5}\left(\mathbf{x}_{f}, t+\delta t\right)=\hat{g}_{7}\left(\mathbf{x}_{f}, t\right)+\kappa_{d} \Phi_{n}, \quad \text { with } \kappa_{r}+2 \kappa_{d}=1 . \\
g_{8}\left(\mathbf{x}_{f}, t+\delta t\right)=\hat{g}_{6}\left(\mathbf{x}_{f}, t\right)+\kappa_{d} \Phi_{n},
\end{array}\right.
$$

A “4-1-1" splitting scheme with $\kappa_{r}=4 / 6$ and $\kappa_{d}=1 / 6$ was proposed by Zhang et al. [40]. We will show that the splitting is not unique; and thus other splitting schemes including $\kappa_{r}=\kappa_{d}=1 / 3$ are also examined in this work.

\subsection{Interpolation based boundary schemes for $0 \leq \Delta \leq 1$}

For a local intersection of the lattice link by the boundary wall with an arbitrary $\Delta$ value, one can combine the (anti-) bounce-back schemes with spatial interpolation of the distribution functions (populations) at the lattice nodes near the boundary to construct second- or higherorder boundary schemes. Li et al. [24] developed second-order accurate Dirichlet and Neumann boundary treatments using three post-collision neighboring values, $\hat{g}_{\alpha}\left(\mathbf{x}_{f}, t\right), \hat{g}_{\alpha}\left(\mathbf{x}_{f f}, t\right)$, and $\hat{g}_{\bar{\alpha}}\left(\mathbf{x}_{f}, t\right)$ for each unknown population $g_{\bar{\alpha}}\left(\mathbf{x}_{f}, t+\delta t\right)$ in the D2Q5 LB model. The "multireflection" schemes in [39] utilize five post-collision populations involving three field nodes, and up to "third-order" accurate boundary schemes were derived for the Dirichlet condition. A detailed study on the numerical accuracy and stability of the second-order Dirichlet and Neumann schemes and their applicability on curved boundaries can be found in [24, 37]. In this work, we also use these interpolated schemes for $0 \leq \Delta \leq 1$ in the D2Q5 model and its extension to D2Q9 models are also discussed in the following. 


\subsubsection{Dirichlet condition treatment}

Following [24], in the interpolation based Dirichlet boundary treatment for both D2Q5 and D2Q9, the single anti-bounce-back population in Eq. (12) is replaced with a combination of three weighted populations

$$
g_{\bar{\alpha}}\left(\mathbf{x}_{f}, t+\delta t\right)=\left[c_{d 1} \hat{g}_{\alpha}\left(\mathbf{x}_{f}, t\right)+c_{d 2} \hat{g}_{\alpha}\left(\mathbf{x}_{f f}, t\right)+c_{d 3} \hat{g}_{\bar{\alpha}}\left(\mathbf{x}_{f}, t\right)\right]+c_{d 4}\left(2 \omega_{\alpha} \Phi_{d}\right),
$$

where $\mathbf{x}_{f f}$ is the second interior lattice node along the $\mathbf{e}_{\bar{\alpha}}$ direction, i.e., $\mathbf{x}_{f f}=\mathbf{x}_{f}+\mathbf{e}_{\bar{\alpha}} \delta t, c_{d 1}-c_{d 4}$ are coefficients related to the local link fraction $\Delta$, and the coefficient $\omega_{\alpha}$ for the D2Q5 and D2Q9 models can be found in Table 1. The asymptotic boundary analysis in [24], which can be directly extended to D2Q9 LB models, showed that second-order accuracy is preserved for the Dirichlet treatment (16) when the following relationship is maintained, with $c_{d 1}(\neq 1)$ an adjustable variable

$$
c_{d 2}=-\frac{2 \Delta c_{d 1}+1}{2 \Delta+1}, c_{d 3}=\frac{c_{d 1}+2 \Delta}{2 \Delta+1}, \text { and } c_{d 4}=\frac{-c_{d 1}+1}{2 \Delta+1} .
$$

Note that the relationships $-c_{d 1}-c_{d 2}+c_{d 3}=1$ and $c_{d 3}+c_{d 4}=1$ hold. The three particular Dirichlet schemes examined in [24] have the following specific choices of $c_{d 1}$

Scheme 1:

$$
c_{d 1}=\left\{\begin{array}{l}
-2 \Delta,(0 \leq \Delta \leq 0.5), \\
-\frac{1}{2 \Delta},(\Delta>0.5)
\end{array}\right.
$$

Scheme 2:

$$
c_{d 1}=-2(1-\Delta), \text { and }
$$

Scheme 3:

$$
c_{d 1}=-1
$$


All three schemes will be applied in this study for both D2Q5 and D2Q9 models. We would like to point out that for certain cases, such as the corner nodes in the D2Q9 lattice, $\mathbf{x}_{f f}$ could be out of the interior domain, and thus the "one-node" second-order Dirichlet scheme can be implemented with $c_{d 1}=-1 /(2 \Delta)$ for $0<\Delta \leq 1$, for which case $c_{d 2}=0$ in Eq. (16).

\subsubsection{Neumann condition treatment}

Similarly, the interpolated Neumann treatment using the same populations is

$$
\begin{array}{r}
\text { D2Q5: } g_{\bar{\alpha}}\left(\mathbf{x}_{f}, t+\delta t\right)=\left[c_{n 1} \hat{g}_{\alpha}\left(\mathbf{x}_{f}, t\right)+c_{n 2} \hat{g}_{\alpha}\left(\mathbf{x}_{f f}, t\right)+c_{n 3} \hat{g}_{\bar{\alpha}}\left(\mathbf{x}_{f}, t\right)\right]+c_{n 4} \Phi_{n \bar{\alpha}}, \alpha=1-4,(19 \mathrm{a}) \\
\text { D2Q9: }\left\{\begin{array}{r}
g_{\bar{\alpha}}\left(\mathbf{x}_{f}, t+\delta t\right)=\left[c_{n 1} \hat{g}_{\alpha}\left(\mathbf{x}_{f}, t\right)+c_{n 2} \hat{g}_{\alpha}\left(\mathbf{x}_{f f}, t\right)+c_{n 3} \hat{g}_{\bar{\alpha}}\left(\mathbf{x}_{f}, t\right)\right]+c_{n 4} \kappa_{r} \Phi_{n}, \alpha=1-4, \\
g_{\bar{\alpha}}\left(\mathbf{x}_{f}, t+\delta t\right)=\left[c_{n 1} \hat{g}_{\alpha}\left(\mathbf{x}_{f}, t\right)+c_{n 2} \hat{g}_{\alpha}\left(\mathbf{x}_{f f}, t\right)+c_{n 3} \hat{g}_{\bar{\alpha}}\left(\mathbf{x}_{f}, t\right)\right]+c_{n 4} \kappa_{d} \Phi_{n}, \alpha=5-8, \\
\text { with } \kappa_{r}+2 \kappa_{d}=1,(19 \mathrm{~b})
\end{array}\right.
\end{array}
$$

For the D2Q5 model, the asymptotic analysis in [24] showed that the coefficients $c_{n 1}-c_{n 4}$ in Eq. (11) are unique for second-order accuracy with

$$
c_{n 1}=1, c_{n 2}=\frac{1-2 \Delta}{2 \Delta+1}, c_{n 3}=\frac{2 \Delta-1}{2 \Delta+1}, \text { and } c_{n 4}=\frac{2}{2 \Delta+1} .
$$

where the relationships $c_{n 1}+c_{n 2}+c_{n 3}=1$ and $c_{n 3}+c_{n 4}=1$ are readily noticed. The same choice for the coefficients is used for the D2Q9 models in this work.

As emphasized in $[24,33]$, when the local boundary normal $\mathbf{n}$ is aligned with $\mathbf{e}_{\bar{\alpha}}$ (Figs. 1a), $\Phi_{n \bar{\alpha}}=\Phi_{n}$ and thus the Neumann scheme (19a) in D2Q5 can be directly applied. When $\mathbf{n}$ is not in the $\mathbf{e}_{\bar{\alpha}}$ direction, which is usually encountered on inclined or curved boundaries, $\Phi_{n \bar{\alpha}}$ is not equal to $\Phi_{n}$ and it also depends on the unknown tangential flux $\Phi_{t}$, i.e., $\Phi_{n \bar{\alpha}}=\Phi_{n} \cos \theta_{n \bar{\alpha}}+\Phi_{t} \sin \theta_{n \bar{\alpha}}$ (see Fig. 2). Since the well-posedness of a mathematical problem 
for CDE does not even allow the specification of $\Phi_{t}$ in addition to $\Phi_{n}$ on the same boundary, a Cartesian decomposition method was proposed in [24] to obtain $\Phi_{n \bar{\alpha}}$ from $\Phi_{n}$ :

$$
\begin{aligned}
\Phi_{n \bar{\alpha}}= & \left\{\frac{1}{c_{d 4}^{\prime}}\left[\left(c_{n 1}^{\prime}-c_{d 1}^{\prime}\right) \hat{g}_{\beta}\left(\mathbf{x}_{f}^{\prime}, t\right)+\left(c_{n 2}^{\prime}-c_{d 2}^{\prime}\right) \hat{g}_{\beta}\left(\mathbf{x}_{f f}^{\prime}, t\right)+\left(c_{n 3}^{\prime}-c_{d 3}^{\prime}\right) \hat{g}_{\bar{\beta}}\left(\mathbf{x}_{f}^{\prime}, t\right)\right] \sin \theta\right. \\
& -\frac{1}{c_{d 4}}\left[\left(c_{n 1}-c_{d 1}\right) \hat{g}_{\alpha}\left(\mathbf{x}_{f}, t\right)+\left(c_{n 2}-c_{d 2}\right) \hat{g}_{\alpha}\left(\mathbf{x}_{f f}, t\right)+\left(c_{n 3}-c_{d 3}\right) \hat{g}_{\bar{\alpha}}\left(\mathbf{x}_{f}, t\right)\right] \sin \theta \\
& \left.+\frac{c_{n 4}^{\prime}}{c_{d 4}^{\prime}} \frac{\delta t}{\delta x} \Phi_{n}\right\} /\left[\frac{c_{n 4}}{c_{d 4}} \frac{\delta t}{\delta x} \sin \theta+\frac{c_{n 4}^{\prime}}{c_{d 4}^{\prime}} \frac{\delta t}{\delta x} \cos \theta\right],
\end{aligned}
$$

where the coefficients $c_{d i}^{\prime}$ and $c_{n i}^{\prime}(i=1,2,3$ and 4) are determined from Eqs. (17) and (20) by setting $\Delta=0$ in the $\boldsymbol{e}_{\bar{\beta}}$ direction, i.e., $c_{d i}^{\prime}=c_{d i}\left(\Delta^{\prime}\right)=c_{d i}(\Delta=0)$, and $c_{n i}^{\prime}=c_{n i}\left(\Delta^{\prime}\right)=c_{n i}(\Delta=0)$; $\hat{g}_{\bar{\beta}}$ and $\hat{g}_{\beta}$ are the post-collision distribution functions along the lattice directions $\mathbf{e}_{\bar{\beta}}$ and $\mathbf{e}_{\beta}$ that are perpendicular to $\mathbf{e}_{\bar{\alpha}}$ and $\mathbf{e}_{\alpha}$, and they are evaluated at the intersection nodes $\mathbf{x}_{f}^{\prime}=\mathbf{x}_{w}$ and $\mathbf{x}_{f f}^{\prime}=\mathbf{x}_{w}+\mathbf{e}_{\bar{\beta}} \delta t$ (see Fig. 2) with interpolation or extrapolation of the neighboring distribution functions. For more details about the Neumann condition treatment in the D2Q5 model for curved boundaries and its extension to mixed boundary conditions please refer to [24].

It should be noted that as reported in $[24,32-34,36,37]$ and also Sec. 4.3 .2 of this work, such a flux decomposition scheme is necessary to obtain first-order accurate LB solutions when using the D2Q5 model. While for the D2Q9 model, construction of such a decomposition scheme is much more complicated due to the additional lattice velocities along the diagonal directions. The numerical results in Sec. 4.3.2 show that when a proper splitting coefficient is selected (e.g., $\kappa_{r}=\kappa_{d}=1 / 3$ ), the Neumann boundary scheme in (19b) is able to yield first-order accurate results with the $\mathrm{D} 2 \mathrm{Q} 9$ model for curved boundaries. 


\subsection{Evaluation of boundary flux/boundary value and interior derivatives}

By combining the Dirichlet and Neumann boundary schemes in Eqs. (16) and (19a), analytical formulas for obtaining the boundary flux/boundary $\phi$ values for given Dirichlet/Neumann problems have been proposed in [24] for D2Q5. For completeness, they are listed below.

The boundary flux for a Dirichlet problem can be evaluated from

D2Q5: $\Phi_{n \bar{\alpha}}=\left(\frac{1-c_{d 1}}{2}\right)\left[-(2 \Delta+1) \hat{g}_{\alpha}\left(\mathbf{x}_{f}, t\right)+\left(2 \Delta-\frac{2}{1-c_{d 1}}\right) \hat{g}_{\alpha}\left(\mathbf{x}_{f f}, t\right)+\left(\frac{2}{1-c_{d 1}}-1\right) \hat{g}_{\bar{\alpha}}\left(\mathbf{x}_{f}, t\right)+2 \omega_{\alpha} \Phi_{d}\right]$,

and the boundary $\phi$ value for a Neumann problem can be evaluated using

D2Q5: $\Phi_{d}=\frac{1}{2 \omega_{\alpha}}\left[(2 \Delta+1) \hat{g}_{\alpha}\left(\mathbf{x}_{f}, t\right)+\left(\frac{2}{1-c_{d 1}}-2 \Delta\right) \hat{g}_{\alpha}\left(\mathbf{x}_{f f}, t\right)+\left(1-\frac{2}{1-c_{d 1}}\right) \hat{g}_{\bar{\alpha}}\left(\mathbf{x}_{f}, t\right)+\left(\frac{2}{1-c_{d 1}}\right) \Phi_{n \bar{\alpha}}\right]$,

where the coefficients in Eqs. $(17,20)$ were used. It is emphasized that $\Phi_{n \bar{\alpha}}$ in Eqs. $(22,23)$ is the boundary flux along the lattice vector $\mathbf{e}_{\bar{\alpha}}$ direction.

Extension of the above technique in D2Q5 to that in D2Q9 is more sophisticated. First, we consider the boundary flux evaluation for a Dirichlet problem with a straight boundary and its normal aligned with the orthogonal lattice vector $\mathbf{e}_{\bar{\alpha}}$ as shown in Fig. 3. For the distributions in the orthogonal lattice direction, i.e., $\alpha=1-4$, the boundary flux $\Phi_{n \bar{\alpha}}$ is the same as the normal flux $\Phi_{n}$ (e.g., on the boundary node $\mathbf{x}_{w 1}$ in Fig. 3). Thus the boundary flux evaluation scheme (see Eq. (24a) below) can be similarly constructed by combining Eqs. (16) and (19b). Based on numerical verification (see Sec. 4.2.1 below), we notice that the particular choice of $\kappa_{r}=4 / 6$ for D2Q9-I, -II and $\kappa_{r}=1 / 3$ for D2Q9-III, -IV is required. It should be noted that the boundary flux can also be obtained from the distributions along the diagonal lattice directions $(\alpha=5-8)$. 
Inspired by the above technique derived in $\mathrm{D} 2 \mathrm{Q} 5$ and the intersection of the lattice links in D2Q9 by the boundary (e.g., $\mathbf{x}_{w 2}$ and $\mathbf{x}_{w 3}$ in Fig. 3), the following scheme for boundary flux evaluation is proposed

$$
\begin{aligned}
& \text { D2Q9: } \Phi_{n}\left(\mathbf{x}_{w 1}\right)=\frac{1}{\kappa_{r}}\left(\frac{\left.1-c_{d 1}\right)}{2}\right)\left[-(2 \Delta+1) \hat{g}_{3}\left(\mathbf{x}_{f 1}, t\right)+\left(2 \Delta-\frac{2}{1-c_{d 1}}\right) \hat{g}_{3}\left(\mathbf{x}_{f f 1}, t\right)\right. \\
& \left.+\left(\frac{2}{1-c_{d 1}}-1\right) \hat{g}_{1}\left(\mathbf{x}_{f 1}, t\right)+2 \omega_{1} \Phi_{d}\left(\mathbf{x}_{w 1}\right)\right] \\
& \text { with } \kappa_{r}=4 / 6 \text { for D2Q9-I, -II, and } \kappa_{r}=1 / 3 \text { for D2Q9-III, -IV. (24a) } \\
& \begin{array}{r}
\text { D2Q9: } \Phi_{n}\left(\mathbf{x}_{w 2,3}\right)=\frac{1}{2 \kappa_{d}}\left(\frac{1-c_{d 1}}{2}\right)\left\{\left[-(2 \Delta+1) \hat{g}_{6}\left(\mathbf{x}_{f 2}, t\right)+\left(2 \Delta-\frac{2}{1-c_{d 1}}\right) \hat{g}_{6}\left(\mathbf{x}_{f f 2}, t\right)\right.\right. \\
\left.+\left(\frac{2}{1-c_{d 1}}-1\right) \hat{g}_{8}\left(\mathbf{x}_{f 2}, t\right)+2 \omega_{8} \Phi_{d}\left(\mathbf{x}_{w 2}\right)\right]+\left[-(2 \Delta+1) \hat{g}_{7}\left(\mathbf{x}_{f 3}, t\right)+\left(2 \Delta-\frac{2}{1-c_{d 1}}\right) \hat{g}_{7}\left(\mathbf{x}_{f f 3}, t\right)\right. \\
\left.\left.+\left(\frac{2}{1-c_{d 1}}-1\right) \hat{g}_{5}\left(\mathbf{x}_{f 3}, t\right)+2 \omega_{5} \Phi_{d}\left(\mathbf{x}_{w 3}\right)\right]\right\}, \\
\text { with } \kappa_{d}=1 / 6 \text { for D2Q9-I, -II, and } \kappa_{d}=1 / 3 \text { for D2Q9-III, -IV. (24b) }
\end{array}
\end{aligned}
$$

The notations for $\mathbf{x}_{f i}$ and $\mathbf{x}_{f f i}(i=1-3)$ are shown in Fig. 3. One can notice from (24b) that $\Phi_{n}\left(\mathbf{x}_{w 2}\right)$ and $\Phi_{n}\left(\mathbf{x}_{w 3}\right)$ are assumed the same and approximated from the average of the distributions along the two discrete lattice vectors. The most accurate result is expected at $\Delta=$ 0.5 , for which $\mathbf{x}_{w 2}=\mathbf{x}_{w 3}$ and the scheme in $(24 \mathrm{a}, \mathrm{b})$ reduces to

$$
\begin{aligned}
& \begin{array}{r}
\text { D2Q9: } \Phi_{n}\left(\mathbf{x}_{w 1}\right)=\frac{1}{\kappa_{r}}\left[-2 \hat{g}_{3}\left(\mathbf{x}_{f 1}, t\right)+2 \omega_{1} \Phi_{d}\left(\mathbf{x}_{w 1}\right)\right], \\
\text { with } \kappa_{r}=4 / 6 \text { for D2Q9-I, -II, and } \kappa_{r}=1 / 3 \text { for D2Q9-III, -IV. (25a) } \\
\text { D2Q9: } \Phi_{n}\left(\mathbf{x}_{w 2}\right)=\Phi_{n}\left(\mathbf{x}_{w 3}\right)=\frac{1}{\kappa_{d}}\left[-\hat{g}_{6}\left(\mathbf{x}_{f 2}, t\right)-\hat{g}_{7}\left(\mathbf{x}_{f 3}, t\right)+2 \omega_{5} \Phi_{d}\left(\mathbf{x}_{w 2}\right)\right], \\
\text { with } \kappa_{d}=1 / 6 \text { for D2Q9-I, -II, and } \kappa_{d}=1 / 3 \text { for D2Q9-III, -IV. (25b) }
\end{array} \\
& \text { We would like to point out that the schemes in Eqs. (24, 25) are necessitated for the purpose of } \\
& \text { comparing the boundary fluxes between D2Q5 and D2Q9; they are only applicable to straight }
\end{aligned}
$$


boundaries. In this work we have no intention to pursue more complex boundary flux evaluation schemes for curved boundaries in D2Q9 since the scheme in D2Q5 has already been demonstrated to be accurate and much simpler.

Second, for the Neumann problem, the method for evaluating boundary $\phi$ in D2Q5 can be directly extended to D2Q9 in all lattice directions. However the choice of $\kappa_{r}$ and $\kappa_{d}$ is not unique,

D2Q9: $\left\{\begin{aligned} \Phi_{d}=\frac{1}{2 \omega_{\alpha}} & {\left[(2 \Delta+1) \hat{g}_{\alpha}\left(\mathbf{x}_{f}, t\right)+\left(\frac{2}{1-c_{d 1}}-2 \Delta\right) \hat{g}_{\alpha}\left(\mathbf{x}_{f f}, t\right)\right.} \\ & \left.+\left(1-\frac{2}{1-c_{d 1}}\right) \hat{g}_{\bar{\alpha}}\left(\mathbf{x}_{f}, t\right)+\left(\frac{2}{1-c_{d 1}}\right) \kappa_{r} \Phi_{n}\right], \alpha=1-4, \\ \Phi_{d}=\frac{1}{2 \omega_{\alpha}} & {\left[(2 \Delta+1) \hat{g}_{\alpha}\left(\mathbf{x}_{f}, t\right)+\left(\frac{2}{1-c_{d 1}}-2 \Delta\right) \hat{g}_{\alpha}\left(\mathbf{x}_{f f}, t\right)\right.} \\ & \left.+\left(1-\frac{2}{1-c_{d 1}}\right) \hat{g}_{\bar{\alpha}}\left(\mathbf{x}_{f}, t\right)+\left(\frac{2}{1-c_{d 1}}\right) \kappa_{d} \Phi_{n}\right], \alpha=5-8,\end{aligned}\right.$

with $\kappa_{r}+2 \kappa_{d}=1$. (26)

For both Dirichlet and Neumann problems, the interior derivatives can be obtained using $[24,41]$

$$
-\tau_{i j} \frac{\partial \phi}{\partial x_{j}}=\frac{1}{\varepsilon_{D} \delta x} \sum_{\alpha=1}^{m-1} e_{\alpha i} g_{\alpha}^{\text {neq }},
$$

where $g_{\alpha}^{\text {neq }}=g_{\alpha}-g_{\alpha}^{\text {eq }}$ is the non-equilibrium component of the distribution function and the constant $\varepsilon_{D}$ is discussed in Eq. (8). Eq. (27) is a local scheme, i.e., it requires only the populations at the local lattice node and is thus more efficient than using finite-difference computations. The second-order accuracy of the scheme has been verified in $[24,32-34,36,37]$ for D2Q5 and [41] for D2Q9 models, where only isotropic diffusion is considered. In this work, fully anisotropic diffusion is also tested and the numerical accuracy of Eq. (27) in both D2Q5 and D2Q9 is investigated. 


\subsection{Total heat/mass transfer on a boundary}

With the boundary flux obtained from Eq. (22) in the D2Q5 LB model, Li et al. [33] proposed a simple and robust scheme for the computation of total flow rate per unit time step $\delta t$ based on the principle of energy conversation. For 2D problems, it was expressed as

$$
\text { D2Q5: } \dot{Q}_{w}=\sum_{w_{b}=1} \sum_{\bar{\alpha} \neq 0}\left[\Phi_{n \bar{\alpha}}(\delta x)^{2}\right] \times\left[1-w\left(\mathbf{x}_{f}+\mathbf{e}_{\alpha} \delta t\right)\right],
$$

where the depth of the boundary is assumed to be one lattice unit $\delta x$ thus the effective surface area for each heat flux $\Phi_{n \bar{\alpha}}$ in the lattice vector direction is $(\delta x)^{2}, w(i, j)$ and $w_{b}(i, j)$ are two scalar arrays with $w(i, j)=1$ for all the field nodes $\mathbf{x}_{f}, w(i, j)=0$ for all the exterior nodes $\mathbf{x}_{e}$, and $w_{b}(i, j)$ is set to zero everywhere except for those field nodes that satisfy $\mathbf{x}_{f}+\mathbf{e}_{\alpha} \delta t=\mathbf{x}_{e}$ (see Figs. 1,2), where a value of 1 is assigned [33]. Eq. (28) requires no surface area approximation and is thus very powerful for heat/mass transfer computations on curved boundaries. Numerical tests were conducted in [33] to demonstrate its second- and first-order accuracy for straight and curved boundaries, respectively.

For the D2Q9 LB models, as discussed in Sec. 3.3, the boundary flux evaluation given by Eqs. (24 and 25) is only applicable to straight walls. Since the surface area can be exactly obtained for straight boundaries, one can directly use Eqs. $(24,25)$ to calculate the total flow rate. The order of accuracy is the same as that for boundary flux evaluation. On the other hand, for curved boundaries, although the local boundary flux cannot be obtained from Eqs. $(24,25)$, the following alternative schemes for the total flow rate computation are proposed in this work following the force evaluation in the hydrodynamic D2Q9 LB model [45, 46]

$$
\text { D2Q9: } \dot{Q}_{w}=\sum_{w_{b}=1} \sum_{\bar{\alpha} \neq 0}\left[\frac{1}{2} \Phi_{n r}(\delta x)^{2}+\frac{1}{4} \Phi_{n d}(\delta x)^{2}\right] \times\left[1-w\left(\mathbf{x}_{f}+\mathbf{e}_{\alpha} \delta t\right)\right],
$$


where $\Phi_{n r}$ and $\Phi_{n d}$ are similar to the respective formulas in Eq. (24a, b), i.e.,

$$
\begin{gathered}
\Phi_{n r}=\frac{1}{\kappa_{r}}\left(\frac{1-c_{d 1}}{2}\right)\left[-(2 \Delta+1) \hat{g}_{\alpha}\left(\mathbf{x}_{f}, t\right)+\left(2 \Delta-\frac{2}{1-c_{d 1}}\right) \hat{g}_{\alpha}\left(\mathbf{x}_{f f}, t\right)\right. \\
\left.+\left(\frac{2}{1-c_{d 1}}-1\right) \hat{g}_{\bar{\alpha}}\left(\mathbf{x}_{f}, t\right)+2 \omega_{\alpha} \Phi_{d}\right], \alpha=1-4, \\
\quad \text { with } \kappa_{r}=4 / 6 \text { for D2Q9-I, -II, and } \kappa_{r}=1 / 3 \text { for D2Q9-III, -IV, (30a) } \\
\Phi_{n d}=\frac{1}{\kappa_{d}}\left(\frac{1-c_{d 1}}{2}\right)\left[-(2 \Delta+1) \hat{g}_{\alpha}\left(\mathbf{x}_{f}, t\right)+\left(2 \Delta-\frac{2}{1-c_{d 1}}\right) \hat{g}_{\alpha}\left(\mathbf{x}_{f f}, t\right)\right. \\
\left.+\left(\frac{2}{1-c_{d 1}}-1\right) \hat{g}_{\bar{\alpha}}\left(\mathbf{x}_{f}, t\right)+2 \omega_{\alpha} \Phi_{d}\right], \alpha=5-8, \\
\text { with } \kappa_{d}=1 / 6 \text { for D2Q9-I, -II, and } \kappa_{d}=1 / 3 \text { for D2Q9-III, -IV. (30b) }
\end{gathered}
$$

The selection of the coefficients $1 / 2$ and $1 / 4$ in Eq. (29) is based on the observation that the effective surface areas for the flux obtained from the distributions in the orthogonal and diagonal lattice directions are $(\delta x)^{2} / 2$ and $(\delta x)^{2} / 4$, respectively (see Fig. 3). This treatment will be validated with a numerical test in Sec. 4.4. For comparison, another treatment with effective surface area $(\delta x)^{2}$ for all flux components in Eq. (30a, b) is also examined, which yields

$$
\text { D2Q9: } \dot{Q}_{w}=\sum_{w_{b}=1} \sum_{\alpha \neq 0}\left[\Phi_{n r}(\delta x)^{2}+\Phi_{n d}(\delta x)^{2}\right] \times\left[1-w\left(\mathbf{x}_{f}+\mathbf{e}_{\alpha} \delta t\right)\right] \text {. }
$$

Realizing that the computation of $\Phi_{n r}$ and $\Phi_{n d}$ in Eq. (30a, b) using three distributions at two lattice nodes should have only first-order accuracy, we can further approximate them by assuming halfway intersection $(\Delta=0.5)$ everywhere such that

$$
\begin{aligned}
& \begin{array}{l}
\Phi_{n r}=\frac{1}{\kappa_{r}}\left[-2 \hat{g}_{\alpha}\left(\mathbf{x}_{f}, t\right)+2 \omega_{\alpha} \Phi_{d}\right], \alpha=1-4, \\
\text { with } \kappa_{r}=4 / 6 \text { for D2Q9-I, -II, and } \kappa_{r}=1 / 3 \text { for D2Q9-III, -IV, (32a) } \\
\Phi_{n d}=\frac{1}{\kappa_{d}}\left[-2 \hat{g}_{\alpha}\left(\mathbf{x}_{f}, t\right)+2 \omega_{\alpha} \Phi_{d}\right], \alpha=5-8, \\
\text { with } \kappa_{d}=1 / 6 \text { for D2Q9-I, -II, and } \kappa_{d}=1 / 3 \text { for D2Q9-III, -IV. (32b) }
\end{array}
\end{aligned}
$$


Lastly, we also consider a treatment that is directly extended from the force evaluation in the hydrodynamic D2Q9 model in [46]. Using the present notations, it is written as

$$
\text { D2Q9: } \begin{aligned}
\dot{Q}_{w} & =\sum_{w_{b}=1} \sum_{\bar{\alpha} \neq 0}\left[\mathbf{e}_{\bar{\alpha}} g_{\bar{\alpha}}\left(\mathbf{x}_{f}, t+\delta t\right)+\mathbf{e}_{\alpha} \hat{g}_{\alpha}\left(\mathbf{x}_{f}, t\right)\right](\delta x)^{2} \times\left[1-w\left(\mathbf{x}_{f}+\mathbf{e}_{\alpha} \delta t\right)\right] \\
& =\sum_{w_{b}=1} \sum_{\bar{\alpha} \neq 0}\left[-2 \hat{g}_{\alpha}\left(\mathbf{x}_{f}, t\right)+2 \omega_{\alpha} \Phi_{d}\right](\delta x)^{2} \times\left[1-w\left(\mathbf{x}_{f}+\mathbf{e}_{\alpha} \delta t\right)\right] .
\end{aligned}
$$

In Sec. 4.4, a numerical test will be presented to show the applicability of the various schemes for the total flow rate computation, including the combinations of $(29,30),(29,32),(31,30)$, and (33). All schemes have a first-order accuracy. While the scheme in (33) is the simplest in terms of implementation, the scheme in Eqs. $(29,30)$ has the best numerical accuracy.

\section{Numerical results and discussion}

The LB results for five numerical tests are presented in this section. The D2Q5 and D2Q9 I-IV models listed in Table 1 are implemented and their accuracy and convergence are compared with each other with reference to the available analytical solution to the CDE in each test. For 3D verification, the D3Q7 and D3Q19 models in the Appendix are implemented. Test 1 is for the convection-diffusion of a Gaussian hill, in which both isotropic and fully anisotropic diffusion at early stage (small times) are considered. Periodic boundary conditions are applied for all sides of the domain in $2 \mathrm{D}$ and $3 \mathrm{D}$ cases in Test 1 so that the effect of boundary condition treatment is excluded. Thus the focus is on the direct comparison of the LB models. Both Dirichlet and Neumann boundary conditions are imposed in each of Tests 2 and 3, of which Test 2 is on the study of the convection-diffusion in a straight $2 \mathrm{D}$ channel with a constant plug flow assumed, while Test 3 is on the study of the pure diffusion in a $2 \mathrm{D}$ circular domain. The performance of D2Q5 and D2Q9 models in the evaluation of total heat/mass transfer rate over a curved boundary 
is investigated in Test 4 . Test 5 is designed to confirm the observations in Test 3 when the D3Q7 and D3Q19 models are applied to simulate pure diffusion in a 3D domain. We assess the accuracy of the interior distribution of $\phi$ and its derivatives in all cases, as well as that of the boundary flux/boundary $\phi$ values in the Dirichlet/Neumann problems, respectively.

\subsection{Transient convection and anisotropic diffusion of a Gaussian hill}

The transient convection-diffusion in a square domain $H=L$ for $2 \mathrm{D}$ and a cubic domain $(H=L=W)$ for $3 \mathrm{D}$ is considered in this test. Assuming an initial condition of Gaussian distribution (a Gaussian hill)

$$
\phi(\mathbf{x}, t=0)=\frac{\phi_{0}}{\left(2 \pi \sigma_{0}^{2}\right)^{d / 2}} \exp \left(-\frac{\mathbf{x}^{2}}{2 \sigma_{0}^{2}}\right),
$$

with $\phi_{0}$ being the total concentration, $\sigma_{0}^{2}$ being the initial variance and $d$ denoting the dimensions, and periodic boundaries on the boundary walls, the exact solution can be obtained $[20,26,41]$

$$
\phi_{\mathrm{ex}}(\mathbf{x}, t)=\frac{\phi_{0}}{(2 \pi)^{d / 2} \sqrt{\left\|\boldsymbol{\sigma}_{t}\right\|}} \exp \left\{-\frac{1}{2} \boldsymbol{\sigma}_{t}^{-1}:[(\mathbf{x}-\mathbf{u} t)(\mathbf{x}-\mathbf{u} t)]\right\},
$$

where $\boldsymbol{\sigma}_{t}=\sigma_{0}^{2} \mathbf{I}+2 t \mathbf{D}, \mathbf{D}$ is the anisotropic diffusion tensor, and $\left\|\boldsymbol{\sigma}_{t}\right\|$ and $\boldsymbol{\sigma}_{t}^{-1}$ are the determinant and inverse of $\boldsymbol{\sigma}_{t}$, respectively. This Gaussian hill convection-diffusion problem is characterized by the Péclet number. For convenience, we choose $D_{22}$ to define the Péclet number as $P e=$ $U H / D_{22}$. For the periodic boundary conditions to hold, a large computational domain is chosen so that for all small but finite times $\boldsymbol{\sigma}_{t}^{-1}:[(\mathbf{x}-\mathbf{u} t)(\mathbf{x}-\mathbf{u} t)] \gg>1$ remains valid at the boundary for $\phi$ to be effectively zero. 
The 2D simulations are discussed first. In the LB computation, $\sigma_{0}^{2}=(0.025 L)^{2}$ is used, the relaxation coefficients $\tau_{i j}$ are determined from Eq. (8), and both isotropic $\left(D_{11}=D_{22}=0.01\right.$, $\left.D_{12}=D_{21}=0\right)$ and fully anisotropic $\left(D_{11}=0.015, D_{12}=0.0025, D_{21}=0.002, D_{22}=0.01\right)$ diffusion cases are considered. The initial treatment for the microscopic distribution functions $g_{\alpha}(\mathbf{x}, t=0)=\left(\omega_{\alpha}+\frac{\delta t u_{j}}{\delta x \varepsilon_{D}} e_{\alpha j} \omega_{\alpha}\right) \phi_{\mathrm{ex}, t=0}-\delta x \frac{\partial \phi_{\mathrm{ex}, t=0}}{\partial x_{j}}\left(\mathrm{M}^{-1} \mathrm{~S}^{-1} \mathrm{M} e_{j} \omega\right)_{\alpha}$, as proposed in [20], is applied here for all the D2Q5 and D2Q9 models. The accuracy of the LB results is evaluated at $t_{m}$ when $\phi_{\max }\left(\mathbf{x}, t_{m}\right)=0.5 \phi_{\max }(\mathbf{x}, 0)$, that is,

$$
t_{m}=\frac{\sigma_{0}^{2}}{4} \frac{\sqrt{\left(D_{11}+D_{22}\right)^{2}+12\|\mathbf{D}\|}-\left(D_{11}+D_{22}\right)}{\|\mathbf{D}\|}
$$

with the determinant $\|\mathbf{D}\|=D_{11} D_{22}-D_{12} D_{21}$. The following $L_{2}$-norm errors are defined to assess the accuracy of the LB results

$$
\begin{gathered}
E_{2}=\left[\sum_{\text {interior nodes }}\left(\phi_{\mathrm{LBE}}-\phi_{\mathrm{ex}}\right)^{2} / \sum_{\text {interior nodes }} \phi_{\mathrm{ex}}^{2}\right]^{1 / 2}, \\
E_{2_{-} \partial \phi / \partial x}=\left\{\sum_{\text {interior nodes }}\left[\left(\tau_{x x} \frac{\partial \phi}{\partial x}+\tau_{x y} \frac{\partial \phi}{\partial y}\right)_{\mathrm{LBE}}-\left(\tau_{x x} \frac{\partial \phi}{\partial x}+\tau_{x y} \frac{\partial \phi}{\partial y}\right)_{\mathrm{ex}}\right]^{2} / \sum_{\text {interior nodes }}\left(\tau_{x x} \frac{\partial \phi}{\partial x}+\tau_{x y} \frac{\partial \phi}{\partial y}\right)_{\mathrm{ex}}^{2}\right\}^{1 / 2}, \\
E_{2_{-} \partial \phi / \partial y}=\left\{\sum_{\text {interior nodes }}\left[\left(\tau_{y x} \frac{\partial \phi}{\partial x}+\tau_{y y} \frac{\partial \phi}{\partial y}\right)_{\mathrm{LBE}}-\left(\tau_{y x} \frac{\partial \phi}{\partial x}+\tau_{y y} \frac{\partial \phi}{\partial y}\right)_{\mathrm{ex}}\right]^{2} / \sum_{\text {interior nodes }}\left(\tau_{y x} \frac{\partial \phi}{\partial x}+\tau_{y y} \frac{\partial \phi}{\partial y}\right)_{\mathrm{ex}}^{2}\right\}^{1 / 2},
\end{gathered}
$$


where $E_{2}$ measures the error of the interior $\phi$ distribution, and $E_{2_{-} \partial \phi / \partial x}, E_{2_{-} \partial \phi / \partial y}$ measures the errors in evaluating the respective partial derivatives using Eq. (27). The values of $(\partial \phi / \partial x)_{\mathrm{ex}}$ and $(\partial \phi / \partial y)_{\mathrm{ex}}$ are calculated directly from the solution in Eq. (35).

Figures 4 (a-f) and 5 (a-f) show the relative $L_{2}$-norm errors defined in Eqs. $(37,38)$, respectively, at $P e=0$ (pure diffusion) and $P e=32,320$ (convection-diffusion with $u_{x}=u_{y}=U$ ) when the D2Q5 and D2Q9 LB models in Table 1 are implemented. The results clearly demonstrate that second-order accuracy is obtained for all cases. It should be noted that for $P e=$ 0, D2Q9-I and D2Q9-II are identical as indicated in Figs. 4(a, b) and 5(a, b), and $E_{2_{-} \partial \phi / \partial x}=$ $E_{2_{-} \partial \phi / \partial y}$ for isotropic diffusion in this test, thus only $E_{2_{-} \partial \phi / \partial x}$ is shown in Fig. 5 (a, c, e). Furthermore, the comparison between the results for D2Q5 and the D2Q9 models implies that in general the D2Q5 model is more accurate in the cases with relatively weak convection at $P e=0$ and 32, while for strong convection cases (e.g., $P e=320$ ), the D2Q5 model gives larger errors and the D2Q9 models seem to have better accuracy. To elucidate this comparison, Fig. 6 (a-d) shows the respective results of $E_{2}$ and $E_{2_{\_} \partial \phi / \partial x}$ versus $P e$ at two different values $\tau_{D}=0.53$ and 0.8 with $\tau_{P}=1$ and $L=64$. The error behavior in the increase of $E_{2}$ and $E_{2_{-} \partial \phi / \partial x}$ with increasing $P e$ is quite similar. For very large $P e$, the numerical error increases considerably and a stable computation would necessitate high resolution. The results in Fig. 6 indicate that in general D2Q5 performs as well as D2Q9 in terms of both numerical accuracy and stability.

To verify that the above observations are equally valid in $3 \mathrm{D}$, the Gaussian hill convection-isotropic diffusion is also simulated in a cube using both D3Q7 and D3Q19 models (see the Appendix for model details). The simulation parameters are kept the same as those for the $2 \mathrm{D}$ isotropic case with $\tau_{D}=0.53$ and $\tau_{P}=1$. Fig. $7(\mathrm{a}, \mathrm{b})$ shows the $L_{2}$-norm errors $E_{2}$ and 
$E_{2 \_o \phi / \partial x}$ defined in Eqs. $(37,38 \mathrm{a})$ when extended to $3 \mathrm{D}$ versus the grid resolution at $t_{m}=\frac{\left(4^{1 / 3}-1\right) \sigma_{0}^{2}}{2 D_{22}}$ when $\phi_{\max }\left(\mathbf{x}, t_{m}\right)=0.5 \phi_{\max }(\mathbf{x}, 0)$. Again, second-order accuracy for both quantities is obtained for each LB model. The change of the error magnitude with $P e$ is also similar to those shown in Figs. 4 and 5; and D3Q7 proves to be more attractive than D3Q19, especially at moderate and low $P e$ values.

It should be emphasized that due to the periodic boundary conditions imposed in this test, the effects of the boundary treatment on the numerical accuracy are eliminated. For general cases, such effects cannot be avoided, and the tests in the following sections will be focused on these issues.

\subsection{Steady convection and isotropic diffusion in a 2D channel}

In this test, the steady convection-diffusion in a 2D channel with imposed Dirichlet or Neumann boundary conditions is examined. This plug flow problem has a simple analytical solution and has been applied in $[24,32-34,36,37,42,43]$ to assess the numerical accuracy and convergence of the proposed boundary and/or conjugate interface schemes. The geometric configuration and the lattice distributions are schematically depicted in Fig. 8. Following [24], Dirichlet or Neumann boundary conditions are imposed on the top and bottom walls, and periodic boundary conditions are assumed in the $x$-direction for both $\phi$ and the distribution function $g_{\alpha}$. Assuming a constant plug flow $\mathbf{u}=(U, 0)$, the characteristic Péclet number reads $P e$ $=U H / D$, where $H$ is the channel height and $D$ is the isotropic diffusion coefficient.

\subsubsection{Dirichlet boundary condition}

With the following Dirichlet boundary conditions imposed on the bottom and top walls

$$
\phi(x, y=0)=\phi(x, y=H)=\cos (k x), \text { with } k=2 \pi / L,
$$


the exact solution is [24]

$$
\phi_{\mathrm{ex}}(x, y)=\operatorname{Real}\left[e^{i k x}\left(\frac{1-e^{-\lambda H}}{e^{\lambda H}-e^{-\lambda H}} e^{\lambda y}-\frac{1-e^{\lambda H}}{e^{\lambda H}-e^{-\lambda H}} e^{-\lambda y}\right)\right],
$$

where "Real" means taking the real part of a complex variable and $\lambda=k \sqrt{1+\frac{i U}{D k}}$.

In addition to the $L_{2}$-norm errors defined in Eqs. $(37,38)$, another $L_{2}$-norm error for the boundary flux is defined following [24]

$$
E_{2_{-} \mathrm{qw}}=\left[\sum_{\text {boundary nodes }}\left[(D \partial \phi / \partial y)_{\mathrm{LBE}}-(D \partial \phi / \partial y)_{\mathrm{ex}}\right]^{2} / \sum_{\text {boundary nodes }}(D \partial \phi / \partial y)_{\mathrm{ex}}^{2}\right]^{1 / 2},
$$

where $(D \partial T / \partial y)_{\mathrm{LBE}}=\Phi_{n}$ is obtained using the flux schemes in Eq. (22) for the D2Q5 model and Eq. (24) for the D2Q9 models, respectively.

Figure 9 (a-c) shows the results for $E_{2}, E_{2 \_} \partial \phi / \partial x$ and $E_{2 \_} \partial \phi / \partial y$, and $E_{2 \_q w}$ defined in Eqs. (37, 38a, b, and 41), respectively, at $P e=0$ and $\Delta=0.5$. The relaxation coefficients $\tau_{x x}=\tau_{y y}=\tau_{D}=$ 0.75 are used for all cases (see Remark III of Sec. 2 for details of the other relaxation coefficients). For the special case of $\Delta=0.5$, the anti-bounce-back schemes in Eq. (13 a-c) are implemented for the Dirichlet condition. Notably, fourth-order accuracy is observed for $E_{2}$, $E_{2 \_} \partial / \partial x$ and $E_{2 \_} \partial \phi / \partial y$ when the D2Q9-I and D2Q9-II models are used (D2Q9-II is identical to D2Q9-I at $P e=0$ ). The other LB models, D2Q5, D2Q9-III and D2Q9-IV, yield second-order accuracy with very close error magnitude as seen from Fig. 9 (a, b). For the boundary flux errors in Fig. 9 (c), all the LB models examined are able to preserve second-order accuracy, and D2Q5 has smaller error magnitude than the D2Q9 models.

It is of great interest to investigate the accuracy of the LB results for more general cases. Figs. 10 and 11 show $E_{2}, E_{2 \_} \partial \phi / \partial x$ and $E_{2 \_} \partial \phi / \partial y$, and $E_{2 \_q w}$ versus $1 / H$ at $P e=0, \Delta=0.25$ and $P e=$ 20, $\Delta=0.5$, respectively. Schemes 1, 2 and 3 (abbreviated as S1, S2, S3 in Fig. 10 (b,c)) 
represent the particular interpolation based Dirichlet schemes with the adjustable parameter determined in Eqs. (18 a-c). Each of the relative $L_{2}$-norm errors exhibits the same convergence order for both $(P e=0, \Delta=0.25)$ and $(P e=20, \Delta=0.5)$. Specifically, second-order accuracy of $E_{2}$ is obtained for all four D2Q9 models I to IV for the interior $\phi_{\mathrm{LBE}}$ distribution; the D2Q9-I, -II and -IV LB models with Dirichlet boundary schemes yield second-order accurate results for $(\partial \phi / \partial y)_{\mathrm{LBE}}$ while the convergence order for $(\partial \phi / \partial x)_{\mathrm{LBE}}$ is 1.5 ; for D2Q9-III, the convergence order is 1.5 for both $(\partial \phi / \partial x)_{\mathrm{LBE}}$ and $(\partial \phi / \partial y)_{\mathrm{LBE}}$; first-order accuracy for the boundary flux $\Phi_{n}$ is observed for all the D2Q9 models; and second-order accuracy is preserved for all the quantities $E_{2}, E_{2 \_} \partial \phi / \partial x, E_{2 \_} \partial \phi / \partial y$, and $E_{2 \_\mathrm{qw}}$ when using the D2Q5 model. The same behavior is confirmed for other cases including $(P e, \Delta)=(0,0.75),(20,0.25)$, and $(200,0.5)$, for which the results are not shown for brevity.

It should be noted that the causes for the different convergence orders between $E_{2} \partial \phi / \partial x$ and $E_{2 \_} \partial \phi / \partial y$ in Figs. 10 (b) and 11 (b) for D2Q9-I, -II and -IV are different. In Fig. 10 (b) with $\Delta_{y}$ $=0.25$, the interpolation based Dirichlet scheme (16) would result in a degraded convergence order of 1.5 for the interior derivative $(\partial \phi / \partial x)_{\mathrm{LBE}}$ in the $x$-direction, which is perpendicular to the intersected lattice link $\Delta_{y}$; while $(\partial \phi / \partial y)_{\mathrm{LBE}}$ is still second-order accurate as periodic boundary conditions are applied in the $x$-direction and $P e=0$. To confirm this observation, we also conduct another numerical test for pure diffusion in a square block $(H=L)$ with Dirichlet boundary conditions on all boundaries, i.e., $\phi(x=0, y)=0.5 \sin (\pi y / H)$ on the left wall and $\phi(x=L, y)=\phi(x, y=0)=\phi(x, y=H)=0$ on the other three walls. The schematic is shown in Fig. 12, and the analytical solution for $\phi$ is $\phi_{\mathrm{ex}}(x, y)=\frac{\sinh [(x / L-L / H) \pi]}{2 \sinh (-\pi L / H)} \sin (\pi y / H)$. The Dirichlet schemes in Eq. (18 a-c) are also implemented for D2Q9-I, -II and -IV, noting that for 
the four corner nodes, $c_{d 1}=-1 /(2 \Delta)$ is used thus $c_{d 2}=0$ and $\hat{g}_{\alpha}\left(\mathbf{x}_{f f}, t\right)$ is not needed to implement the second-order Dirichlet scheme in Eq. (16) when $\mathbf{x}_{f f}$ is in the exterior of the block. Using the same LB simulation parameters as in the plug flow problem at $P e=0$, the orders of convergence, denoted as $P_{x}$ and $P_{y}$, for the respective $E_{2 \_} \partial \phi / \partial x$ and $E_{2 \_} \partial \phi / \partial y$ are $\left(P_{x}, P_{y}\right)=(1.5,2)$ at $\left(\Delta_{x}, \Delta_{y}\right)=(0.5,0.25),\left(P_{x}, P_{y}\right)=(2,1.5)$ at $\left(\Delta_{x}, \Delta_{y}\right)=(0.25,0.5)$, and $\left(P_{x}, P_{y}\right)=(1.5,1.5)$ at $\left(\Delta_{x}\right.$, $\left.\Delta_{y}\right)=(0.25,0.25)$, confirming the observed convergence orders in Fig. 10 (b). On the other hand, the lower convergence order of 1.5 for $E_{2} \partial \phi / \partial x$ than second-order for $E_{2 \_} \partial \phi / \partial y$ in Fig. 11 (b) is due to the effect of convection in the $x$-direction in this channel flow problem at $P e>0$. It is worth noting that although second-order convergence was reported in [41] for both the interior flux in the $x$ - and $y$-directions, the examined thermal couette flow and planar thermal Poiseuille flow problems have no variations in the $x$-direction, resulting in $\partial \phi / \partial x=0$ and the flux in the $x$ direction is essentially the convection flux $(U \phi)$ that is only related to the interior $\phi$ values. Thus the present results are not inconsistent with those reported in [41] for the interior $\phi$ values and derivatives.

Furthermore, similar to the results in Fig. 6, the variations of $E_{2}, E_{2 \_} \partial \phi / \partial x$ and $E_{2 \_} \partial \phi / \partial y$, and $E_{2 \_ \text {qw }}$ with $P e$ are shown in Fig. 13 (a-c) at $H=64, \Delta=0.5$ and $\left(\tau_{D}, \tau_{P}\right)=(0.75,1.0)$. The results in Fig. 13 demonstrate that for convection-diffusion problems with specific Dirichlet boundary conditions, the D2Q5 LB model has better numerical accuracy compared to the D2Q9 models examined.

The above results for the D2Q5 and D2Q9 LB models in Table 1 for convection-diffusion problems with Dirichlet conditions on straight boundaries can be summarized as follows. For the very special case $P e=0$ and $\Delta=0.5$, i.e., pure diffusion with the boundary located "halfway" between two lattice nodes, the D2Q9-I and -II models are able to give fourth-order accuracy 
interior distribution of $\phi$ and its derivatives $\partial \phi / \partial x$ and $\partial \phi / \partial y$, while D2Q9-III and -IV have second-order accuracy for both quantities. The boundary flux scheme yields second-order accuracy for all LB models considered. For general cases, both the placement of the boundary in the lattice and the convection affect the orders of accuracy of the interior derivatives and the boundary flux when using the D2Q9 models. Nevertheless, second-order accuracy for the $\phi$ field is always preserved for the $\mathrm{D} 2 \mathrm{Q} 9$ models when the interpolation based Dirichlet schemes are implemented. Consistent with [24], the D2Q5 model is able to preserve second-order accuracy for all three quantities for all cases. Compared with the results in Fig. 6, where periodic boundary conditions were used, the increase of the numerical errors with the Péclet number in Fig. 13 also demonstrates that for a wide range of $P e$ values examined, the D2Q5 model has better numerical accuracy than the D2Q9 models when a Dirichlet condition is implemented.

\subsubsection{Neumann boundary condition}

The above channel flow problem is also ideal for examining the effect of Neumann boundary schemes on the performance of various LB models. By replacing the boundary conditions in Eq. (32) with the following and keeping the other conditions the same

$$
\left.\frac{\partial \phi}{\partial y}\right|_{y=0, H}=\mp \cos (k x) / H,
$$

the exact solution becomes [24]

$$
\phi_{\mathrm{ex}}(x, y)=\operatorname{Real}\left\{e^{i k x}\left[\frac{e^{-\lambda H}+1}{\lambda H\left(e^{\lambda H}-e^{-\lambda H}\right)} e^{\lambda y}+\frac{e^{\lambda H}+1}{\lambda H\left(e^{\lambda H}-e^{-\lambda H}\right)} e^{-\lambda y}\right]\right\} .
$$

In addition to the $L_{2}$-norm errors for the interior $\phi$ and its derivatives in Eqs. $(37,38)$, the relative $L_{2}$-norm error for the boundary $\phi$ values is defined following [24]

$$
E_{2 \_\phi \mathrm{W}}=\left[\sum_{\text {boundary nodes }}\left(\phi_{\mathrm{LBE}}-\phi_{\mathrm{ex}}\right)^{2} / \sum_{\text {boundary nodes }} \phi_{\mathrm{ex}}^{2}\right]^{1 / 2},
$$


where $\phi_{\mathrm{LBE}}$ at the boundary nodes are obtained using the schemes in Eq. (23) for D2Q5 and Eq. (26) for D2Q9.

The Neumann boundary schemes in Eq. (19a, b) for the D2Q5 and D2Q9 LB models are implemented (they reduce to the respective "bounce-back" schemes in Eqs. $(14,15)$ at $\Delta=0.5)$. Figs. 14-16 show the results for $E_{2}, E_{2 \_} \partial \phi / \partial x$ and $E_{2 \_\alpha \phi / \partial y}$, and $E_{2 \_\phi w}$ defined in Eqs. $(37,38 \mathrm{a}, \mathrm{b}$, and 44), respectively, at different $P e$ and $\Delta$ values. In all cases, the flux splitting weights are $\kappa_{r}=4 / 6$ for D2Q9-I, -II and $\kappa_{r}=1 / 3$ for D2Q9-III, -IV with $\kappa_{r}+2 \kappa_{d}=1$ enforced for all four models. The coefficient in Scheme 2 is used for the adjustable parameter $c_{d 1}$ when computing the boundary values using Eqs. $(23,26)$ at $\Delta \neq 0.5$. Clearly, first-order accuracy is obtained for $E_{2}, E_{2 \_} \partial \phi / \partial y$, and $E_{2_{-} \phi \mathrm{w}}$, and the convergence order is 0.5 for $E_{2_{-} \partial \phi / \partial x}$, for each case in Figs. 14-16 when using the four D2Q9 models. On the other hand, the D2Q5 model is able to preserve second-order accuracy for all the quantities examined, which is consistent with the results reported in [24]. Compared to the results in Figs. $10(a, b)$ and $11(a, b)$ for the Dirichlet problem, the order of accuracy for both the interior distribution $\phi$ and its derivatives is degraded by one degree in Figs. $14(b, c)$ and $15(b, c)$ for D2Q9 models. It should also be noted that the magnitude of the relative errors in the D2Q5 model is generally much smaller than that in the D2Q9 models.

To elucidate the effect of the flux splitting weights $\kappa_{r}$ and $\kappa_{d}$ (see Eqs. $(15,19 \mathrm{~b})$ ) on the accuracy and convergence order of the D2Q9 LB results, Fig. 17 (a-c) shows $E_{2}, E_{2} \partial \phi / \partial x$ and $E_{2_{-} \partial \phi / \partial y}$, and $E_{2_{-} \phi \mathrm{w}}$, respectively, versus $1 / H$ at $P e=20$ and $\Delta=0.5$ when using different weights $\left(\kappa_{r}+2 \kappa_{d}=1\right.$ is maintained) for D2Q9-I. Very close results are noticed for the other three D2Q9 models, and the same behavior is checked for different $P e$ and $\Delta$ values; thus they are not shown for brevity. It is obvious that the specific choice of the splitting weights $\kappa_{r}$ and $\kappa_{d}$ has little effect on the numerical accuracy and the same convergence orders are obtained for all different 
choices. This confirms that the splitting weights in the Neumann schemes in D2Q9 are not unique.

\subsection{Steady isotropic diffusion in a $2 \mathrm{D}$ circle}

A curved boundary has a significant effect on the accuracy and convergence order of the LB results due to the irregular distribution of the intersection $\Delta$ values along the boundary [20, 24, 32-37, 42]. The interpolation based Dirichlet and Neumann boundary schemes are capable of preserving the curved geometry when the local $\Delta$ values along each intersection link direction are prescribed. The applicability and numerical accuracy of the D2Q5 model along with the Dirichlet and Neumann schemes have been investigated in [24]. Here we also apply various D2Q9 models in Table 1 to study the same isotropic diffusion problem in a 2D circular domain. The geometry and lattice distribution are depicted in Fig. 18.

\subsubsection{Dirichlet boundary condition}

With a steady Dirichlet boundary condition on the wall

$$
\phi(r=R)=f(\varphi)=\cos (n \varphi), n=\text { integer }
$$

the exact solution for the pure isotropic diffusion problem is [24]

$$
\phi_{\mathrm{ex}}(r, \varphi)=(r / R)^{n} \cos (n \varphi)
$$

The interpolation based Dirichlet boundary scheme in Eq. (16) with the adjustable parameter determined in Scheme 2 (Eq. (18b)) is implemented for D2Q5 and all four D2Q9 LB models listed in Table 1. A relaxation coefficient $\tau_{D}=0.75$ and $n=4$ are used for both the Dirichlet and Neumann problems in this test. Fig. 19 (a, b) shows the $L_{2}$-norm errors $E_{2}$ and $E_{2 \_} \partial \phi / \partial x$ defined in Eqs. $(37,38)$ for the interior distribution of $\phi$ and its derivatives $\left(E_{2 \_} \partial \phi / \partial y=\right.$

$\left.E_{2 \_} \partial \phi / \partial x\right)$, respectively. It is observed that second-order accuracy of the interior $\phi$ field is preserved for all the LB models, and the D2Q9-I, -II (identical for pure diffusion problems) models have 
better accuracy than the D2Q5 and D2Q9-III, -IV models; a convergence order of 1.5 is obtained for the interior derivatives. The orders of convergence for the D2Q9 models are consistent with those in the above channel flow Dirichlet problem and the block diffusion Dirichlet problem at $P e=0$ and $\Delta \neq 0.5$. For D2Q5, the order of accuracy for the interior derivatives is degraded due to the curved boundary [24].

It should be noted that since the local boundary flux evaluation scheme in Eq. (24) for the D2Q9 models is only applicable to straight boundaries, the results for $E_{2 \_q w}$ is not available for the D2Q9 models in this problem with a curved boundary. In contrast, as reported in [24], the boundary flux in the lattice directions can be obtained in the D2Q5 model using the scheme in Eq. (22) with first-order accuracy for $E_{2 \_q w}$. Therefore, the D2Q5 model still proves to be a better choice than the D2Q9 models for Dirichlet problems with curved boundaries.

\subsubsection{Neumann boundary condition}

For the Neumann problem, one can replace the boundary condition in Eq. (45) with

$$
\partial \phi /\left.\partial r\right|_{r=R}=n \cos (n \varphi) / R
$$

which yields the same analytical solution as given by Eq. (46).

Figure 20 (a-c) shows the $L_{2}$-norm errors, $E_{2}, E_{2 \_} \partial \phi / \partial x$, and $E_{2 \_\phi w}$ defined in Eqs. $(37,38$, 44), respectively, versus the grid resolution, 1/R. The Neumann boundary schemes include Eq. (19a) for D2Q5 with the Cartesian decomposition method given by Eq. (21) to obtain $\Phi_{n \bar{\alpha}}$, and the normal flux splitting scheme in Eq. (19b) with $\kappa_{r}=\kappa_{d}=1 / 3$ for D2Q9 I-IV. Again, the coefficient in Scheme 2 is used for the adjustable parameter $c_{d 1}$ when computing the boundary $\phi_{w}$ values using Eqs. $(23,26)$ at $\Delta \neq 0.5$.

Notably, the magnitude of the relative errors is substantially smaller when using the D2Q5 model compared to the D2Q9 models in each figure. In addition, first-order convergence 
is obtained for both the interior and boundary $\phi$ values (Fig. 20 (a, c)) when D2Q5, D2Q9-I and D2Q9-II are applied; while the relative errors converge to a magnitude close to 0.1 for $1 / R<0.04$ for both $E_{2}$ and $E_{2 \_ \text {фw }}$ when using D2Q9-III and D2Q9-IV, indicating that the D2Q9-I and D2Q9II LB models are more robust than D2Q9-III and D2Q9-IV. Furthermore, a convergence order of 0.5 for the interior derivative is observed for all four D2Q9 models, which is consistent with that in Figs. 15 and 17 for the channel flow Neumann problem. The convergence order of 1.5 for $E_{2 \_} \partial \phi / \partial x$ for D2Q5 is consistent with that reported in [24].

\subsection{Transient isotropic diffusion in a 2D circle}

In this test we consider transient isotropic diffusion in a circle as shown in Fig. 18 to compare the total heat/mass transfer evaluation using the Schemes given by Eq. (28) for D2Q5 and Eq. (29) for D2Q9. This problem has been studied in [24] to verify the Dirichlet boundary scheme for transient problems and in [33] to verify the total heat transfer evaluation in the D2Q5 model. The transient Dirichlet condition reads

$$
T(t, r=R)=f(t)=\sin (\omega t),
$$

where $\omega$ is the frequency of the oscillation. This transient problem is characterized by the Stokes number, $S t$, defined as $S t^{2}=\frac{R^{2} \omega}{2 \pi D}$. With no variation in the azimuthal direction, the analytical solution for this 2-D problem can be obtained as [24]

$$
\begin{aligned}
\phi_{\mathrm{ex}}(r, t) & =f(t)-\frac{2}{R} \sum_{n=1}^{\infty} \frac{J_{0}\left(\beta_{n} r\right)}{\beta_{n} J_{1}\left(\beta_{n} R\right)}\left[f(0) e^{-D \beta_{n}^{2} t}+\int_{0}^{t} e^{-D \beta_{n}^{2}(t-\tau)} d f(\tau)\right] \\
& =\sin (\omega t)-\frac{2}{R} \sum_{n=1}^{\infty} \frac{\omega J_{0}\left(\beta_{n} r\right)}{\beta_{n} J_{1}\left(\beta_{n} R\right)}\left[\frac{D \beta_{n}^{2} \cos (\omega t)+\omega \sin (\omega t)-D \beta_{n}^{2} e^{-D \beta_{n}^{2} t}}{\left(D \beta_{n}^{2}\right)^{2}+\omega^{2}}\right],
\end{aligned}
$$

where $J_{i}(\beta r)(i=0,1)$ is the Bessel's function of the first kind of order $i$, and $\beta_{n}$ is determined from the roots of $J_{0}\left(\beta_{n} R\right)=0$. 
Following [33], we defined the following error norm to assess the heat transfer evaluation schemes

$$
E_{2}=\frac{1}{r_{0}} \frac{\omega}{2 \pi} \int_{0}^{2 \pi / \omega}\left|\dot{Q}_{w_{-} \mathrm{LBE}}\left(t^{*}\right)-\dot{Q}_{w_{-} \mathrm{ex}}\left(t^{*}\right)\right| d t=\frac{1}{r_{0}} \int_{0}^{1}\left|\dot{Q}_{w_{-} \mathrm{LBE}}\left(t^{*}\right)-\dot{Q}_{w_{-} \mathrm{ex}}\left(t^{*}\right)\right| d t^{*}
$$

where $t^{*}=\omega t / 2 \pi, \dot{Q}_{w_{-} \text {LBE }}$ is obtained from Eq. (28) for the D2Q5 model and Eqs. $(29,31,33)$ for the D2Q9 models. To eliminate the effect of the initial variations, the computation is performed for a sufficiently long period of time $\left(t^{*}>>1\right)[34,33]$. After the dynamic steady state is reached, the average error $E_{2}$ defined in Eq. (50) is computed and the results are shown in Fig. 21. The Stokes number $S t$ is set to be unity, and the coefficient $c_{d 1}$ determined from Scheme 2 in Eq. (18b) is used for all cases. The presented data are for D2Q9-I (identical to D2Q9-II without convection); very close results are noticed for D2Q9-III and D2Q9-IV, and thus they are not shown.

The results in Fig. 21 clearly show that for a curved boundary, the total flow rate computation in both D2Q5 and D2Q9 models has first-order accuracy. The interpolation based scheme in Eqs. $(29,30)$ proves to be the most stable and accurate one for D2Q9. The antibounce-back based schemes in Eqs. $(29,32)$ and (33) have large oscillations and the average of the error magnitude is close to that in $(29,30)$. The scheme from using Eqs. $(31,30)$ has significantly larger errors (about two orders of magnitude higher in Fig. 21). This comparison clearly demonstrates that the effective surface area with $(\delta x)^{2} / 2$ and $(\delta x)^{2} / 4$ for the respective $\Phi_{n r}$ and $\Phi_{n d}$ in Eq. (30a, b) is a much better choice for D2Q9 models. Nevertheless, the comparison between the results using $\mathrm{D} 2 \mathrm{Q} 5$ and $\mathrm{D} 2 \mathrm{Q} 9$ shows that the $\mathrm{D} 2 \mathrm{Q} 5$ model is still more attractive than the much more complex D2Q9 models in evaluating the total heat/mass transfer over a closed curved boundary. 


\subsection{Steady isotropic diffusion in a 3D sphere}

The foregoing comparisons demonstrate that the simpler D2Q5 LB model with just orthogonal lattice vectors performs as well as or better than the D2Q9 models that include the diagonal lattice vectors. To demonstrate that the same conclusions apply to 3D LB models for scalar convection-diffusion problems with curved geometry, the steady isotropic diffusion in a sphere is considered. The 2D lattice layout and computational domain in Fig. 18 is extended to 3D, and Fig. 18 represents the lattice distribution in the central cross-section on the $x-y$ plane. An axisymmetric Dirichlet boundary condition of a Legendre polynomial $P_{n}(\mu=\cos \theta)$ with respect to the vertical $y$-axis is imposed on the sphere surface, $r=R$, where $\theta$ is the azimuthal angle. Thus the analytical solution is [34]

$$
\phi_{\mathrm{ex}}(r, \mu)=(r / R)^{n} P_{n}(\mu)
$$

A representative case of $P_{4}(\mu)=\left(35 \mu^{4}-30 \mu^{2}+3\right) / 8$ is used in the simulations. When the local lattice link fractions are calculated, the interpolation based Dirichlet schemes are implemented.

Figure $22(\mathrm{a}, \mathrm{b})$ shows the $L_{2}$-norm errors, $E_{2}$ and $E_{2 \_} \partial \phi / \partial x i$, for the interior distribution of $\phi$ and its derivatives defined in Eqs. $(37,38)$ that are extended to the $3 \mathrm{D}$ isotropic case. Note

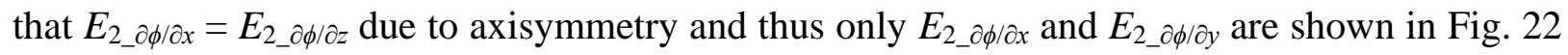
(b). Realizing the similar performance of the various D2Q9 models in Sec. 4.3.1 with curved geometry, only one D3Q19 model that is directly extended from D2Q9-I in Table 1 is chosen for demonstration, and the relaxation coefficient is again $\left(\tau_{D}, \tau_{P}\right)=(0.75,1.0)$. Clearly, secondorder accuracy for the distribution of $\phi$ and a superlinear convergence order of 1.5 for the interior derivatives are obtained. The results are consistent with those in Fig. 19 for the 2D problem. It is important to note that the error magnitudes for the D3Q7 and D3Q19 models are 
very close to each other. Recalling that D3Q7 is significantly less expensive than D3Q19, the preference of D3Q7 over D3Q19 becomes obvious.

\section{Conclusions}

The comparison between the D2Q5 and D2Q9 lattice Boltzmann (LB) models for the convection-diffusion equation (CDE) for scalar transport is investigated. Similar observations are confirmed by comparing the performance of D3Q7 and D3Q19 LB models for 3D problems. For the D2Q5 model, there is essentially one tunable constant parameter when the high-order velocity terms are neglected. Four different D2Q9 models are used and the results are directly compared with those from the D2Q5 model. With the additional diagonal lattice velocities, boundary condition treatment for the D2Q9 LB model is more complex than that for D2Q5. The interpolation based second-order accurate Dirichlet and Neumann boundary treatments that have been verified in D2Q5 are extended to D2Q9. It is confirmed that the Dirichlet treatment is directly applicable while the Neumann treatment should be combined with a normal flux splitting scheme with adjustable weights in D2Q9.

Several representative numerical tests with analytical solutions are conducted to assess the performance of the D2Q5 and D2Q9 LB models in terms of the numerical accuracy and convergence orders of the interior distribution of the scalar variable $\phi$ and its derivatives as well as the boundary flux, total flow rate through the boundary, and boundary scalar values.

First, the second-order accuracy for $\phi$ and its derivatives is verified for both D2Q5 and D2Q9 in the Gaussian hill convection-anisotropic diffusion problem with periodic boundary conditions where the boundary effect is eliminated. The D2Q5 model shows smaller errors when 
the convection is weak or moderate, while the D2Q9 models have better accuracy when the convection is very strong.

Second, for the CDE with specific boundary conditions on straight walls, the D2Q5 model with the interpolation based boundary schemes is able to preserve the second-order accuracy for all the quantities of interest in both Dirichlet and Neumann problems. For the D2Q9 models, second- and first-order accurate interior distribution of $\phi$ for the Dirichlet and Neumann problems are obtained, respectively; the convergence orders for the interior derivatives are degraded to 1.5 ("superlinear") in the Dirichlet problem and 0.5 ("sublinear") in the Neumann problem; and only first-order accurate boundary flux/boundary values are obtained for the Dirichlet/Neumann problems.

Third, the diffusion problem in a circle with a curved boundary demonstrates that (i) for the Dirichlet problem, both the D2Q5 and D2Q9 models have second-order accuracy for the interior distribution of $\phi$, a superlinear (order 1.5) convergence for its interior derivatives, and a first-order convergence for the overall heat/mass transfer; the local boundary flux evaluation scheme in D2Q5 still has first-order accuracy while it is not directly applicable to the D2Q9 models; and (ii) for the Neumann problem, first-order accurate interior and boundary $\phi$ values are obtained for both the D2Q5 and D2Q9 models, while the convergence orders for the interior derivatives are 1.5 and 0.5 for D2Q5 and D2Q9, respectively; more importantly, the error magnitude in the D2Q5 model is much smaller compared to that in the D2Q9 models in the Neumann problem. The simulation of diffusion in a sphere with the D3Q7 and D3Q19 models confirms the comparison between the D2Q5 and D2Q9 models for scalar transport with curved geometry. 
It is concluded that the D2Q5 (D3Q7) LB model is more robust and accurate than the D2Q9 (D3Q19) LB models for the CDE when the convection is not very strong and the boundary effect is significant. It takes less computational resources and is more convenient in handling curved boundaries.

\section{Acknowledgements}

This paper was prepared with the support of the U.S. Department of Energy, ARPA-E, under Award No. DEAR0000184.

\section{References}

[1] G.R. McNamara, G. Zanetti, Use of the Boltzmann equation to simulate lattice-gas automata, Phys. Rev. Lett. 61 (1988) 2332-2335.

[2] F.J. Higuera, S. Succi, R. Benzi, Lattice gas dynamics with enhanced collisions, Europhys. Lett., 9 (1989), 345-349.

[3] R. Benzi, S. Succi, M. Vergassola, The lattice Boltzmann equation: theory and applications, Phys. Rep. 222 (1992), 145-197.

[4] S. Chapman, T. Cowling, The mathematical theory of non-uniform gases (Cambridge University Press) 1990.

[5] X. He, L.-S. Luo, Lattice Boltzmann model for the incompressible Navier-Stokes equation, J. Stat. Phys. 88 (1997) 927-944.

[6] S. Chen, G.D. Doolen, Lattice Boltzmann method for fluid flows, Annu. Rev. Fluid Mech. 30 (1998) 329-364.

[7] M. Junk, A. Klar, L.-S. Luo, Asymptotic analysis of the lattice Boltzmann equation, $J$. Comput. Phys. 210 (2005) 676-704.

[8] R. Mei, W. Shyy, D. Yu, L.-S. Luo, Lattice Boltzmann method for 3-D flows with curved boundary, J. Comput. Phys. 161 (2000) 680-699.

[9] D. d'Humières, I. Ginzburg, M. Krafczyk, P. Lallemand, L.-S. Luo, Multiple-relaxationtime lattice Boltzmann models in three dimensions, Phil. Trans. R. Soc. Lond. A 360 (2002) 4437-451.

[10] F.J. Alexander, S. Chen, J.D. Sterling, Lattice Boltzmann thermohydrodynamics, Phys. Rev. E 47 (1993) R2249. 
[11] X. Shan, Solution of Rayleigh-Benard convection using a lattice Boltzmann method, Phys. Rev. E 55 (1997) 2780.

[12] Y. Chen, H. Ohashi, M. Akiyama, Thermal lattice Bhatnagar-Gross-Krook model without nonlinear deviations in macrodynamic equations, Phys. Rev. E 50 (1994) 2776.

[13] P. Lallemand, L.-S. Luo, Theory of the lattice Boltzmann method: Acoustic and thermal properties in two and three dimensions, Phys. Rev. E 68 (2003) 036706.

[14] X. He, S. Chen, G.D. Doolen, A novel thermal model for the lattice Boltzmann method in incompressible limit, J. Comput. Phys. 146 (1998), 282-300.

[15] R.G.M. van der Sman, M.H. Ernst, Convection-diffusion lattice Boltzmann scheme for irregular lattices, J. Comput. Phys. 160 (2000), 766-782.

[16] Z. Guo, C. Zheng, B. Shi, Thermal lattice Boltzmann equation for low Mach number flows: decoupling model, Phys. Rev. E 75 (2007), 036704.

[17] B. Servan-Camas, F. T.-C. Tsai, Lattice Boltzmann method with two relaxation times for advection-diffusion equation: Third order analysis and stability analysis, Adv. Water Resour. 31 (2008) 1113-1126.

[18] B.C. Shi, Z. Guo, Lattice Boltzmann model for nonlinear convection-diffusion equations, Phys. Rev. E 79 (2009) 016701.

[19] A. Mezrhab, M.A. Moussaoui, M. Jami, H. Naji, M. Bouzidi, Double MRT thermal lattice Boltzmann method for simulating convective flows, Phys. Lett. A 374 (2010), 3499-3507.

[20] H. Yoshida, M. Nagaoka, Multiple-relaxation-time lattice Boltzmann model for the convection and anisotropic diffusion equation, J. Comput. Phys. 229 (2010), 7774-7795.

[21] I. Ginzburg, D. d'Humières, A. Kuzmin, Optimal stability of advection-diffusion lattice Boltzmann models with two relaxation times for positive/negative equilibrium, J. Stat. Phys. 139 (2010), 1090-1143.

[22] I. Ginzburg, Multiple anisotropic collisions for advection-diffusion Lattice Boltzmann schemes, Adv. Water Resour. 51 (2013), 381-404.

[23] Z. Chai, T.S. Zhao, Lattice Boltzmann model for the convection-diffusion equation, Phys. Rev. E 87 (2013), 063309.

[24] L. Li, R. Mei, J.F. Klausner, Boundary conditions for thermal lattice Boltzmann equation method, J. Comput. Phys. 237 (2013), 366-395.

[25] J. Wang, D. Wang, P. Lallemand, L.-S. Luo, Lattice Boltzmann simulations of thermal convective flows in two dimensions, Comput. Mathe. Appli. 65 (2013), 262-286.

[26] R. Huang, H. Wu, A modified multiple-relaxation-time lattice Boltzmann model for convection-diffusion equation, J. Comput. Phys. 274 (2014), 50-63.

[27] P.L. Bhatnagar, E.P. Gross, M. Krook, A model for collision processes in gases. I. small amplitude processes in charged and neutral one-component systems, Phys. Rev. 94 (1954) 511-525.

[28] I. Ginzburg, Lattice Boltzmann modeling with discontinuous collision components. Hydrodynamic and advection-diffusion equations. J. Stat. Phys. 126 (2007), 157-203. 
[29] P. Lallemand, L.-S. Luo, Theory of the lattice Boltzmann method: dispersion, dissipation, isotropy, Galilean invariance, and stability, Phys. Rev. E 61, 6546-6562 (2000).

[30] D. Yu, R. Mei, L.-S. Luo, W. Shyy, Viscous flow computations with the method of lattice Boltzmann equation, Prog. Aerosp. Sci. 39, 329-367 (2003).

[31] D. Contrino, P. Lallemand, P. Asinari, L.-S. Luo, Lattice-Boltzmann simulations of the thermally driven 2D square cavity at high Rayleigh numbers, J. Comput. Phys. 275 (2014), 257-272.

[32] L. Li, C. Chen, R. Mei, J.F. Klausner, Conjugate heat and mass transfer in the lattice Boltzmann equation method, Phys. Rev. E 89 (2014), 043308.

[33] L. Li, R. Mei, J.F. Klausner, Heat transfer evaluation on curved boundaries in thermal lattice Boltzmann equation method, ASME J. Heat Transfer 136 (2014), 012403.

[34] L. Li, R. Mei, J.F. Klausner, Multiple-relaxation-time lattice Boltzmann model for the axisymmetric convection diffusion equation, Int. J. Heat Mass Transfer 67 (2013), 338351.

[35] H. Yoshida, T. Kobayashi, H. Hayashi, T. Kinjo, H. Washizu, K. Fukuzawa, Boundary condition at a two-phase interface in the lattice Boltzmann method for the convectiondiffusion equation, Phys. Rev. E 90 (2014), 013303.

[36] K. Guo, L. Li, G. Xiao, N. AuYeung, R. Mei, Lattice Boltzmann method for conjugate heat and mass transfer with interfacial jump conditions, Int. J. Heat Mass Transfer 88 (2015), 306-322.

[37] L. Li, N. AuYeung, R. Mei, J.F. Klausner, Effects of tangential-type boundary condition discontinuities on the accuracy of lattice Boltzmann method for heat and mass transfer, Phys. Rev. E, 94, 023307 (2016).

[38] D. d'Humieres, I. Ginzburg, Viscosity independent numerical errors for lattice Boltzmann models: from recurrence equations to "magic" collision numbers. Comp. Math. Appl. 58 (2009), 823-840.

[39] I. Ginzburg, Generic boundary conditions for lattice Boltzmann models and their application to advection and anisotropic-dispersion equations. Adv. Wat. Res. 28 (2005), 1196-1216.

[40] X. Zhang, J.W. Crawford, A.G. Bengough, I.M. Young, On boundary conditions in the lattice Boltzmann model for advection and anisotropic dispersion equation, Adv. Wat. Res. 25 (2002) 601-609.

[41] Z. Chai, T.S. Zhao, Nonequilibrium scheme for computing the flux of the convectiondiffusion equation in the framework of the lattice Boltzmann method, Phys. Rev. E 90 (2014) 013305.

[42] G. Le, O. Oulaid, J. Zhang, Counter-extrapolation method for conjugate interfaces in computational heat and mass transfer, Phys. Rev. E 91 (2015) 033306.

[43] H. Karani, C. Huber, Lattice Boltzmann formulation for conjugate heat transfer in heterogeneous media, Phys. Rev. E 91 (2015) 023304. 
[44] X. He, L.-S. Luo, Theory of the lattice Boltzmann method: From the Boltzmann equation to the lattice Boltzmann equation, Phys. Rev. E 56 (1997) 6811.

[45] A.J.C. Ladd, Numerical simulations of particulate suspensions via a discretized Boltzmann equation. Part 2. Numerical results, J. Fluid Mech. 271 (1994) 311-339.

[46] R. Mei, D. Yu, W. Shyy, L.-S. Luo, Force evaluation in the lattice Boltzmann method involving curved geometry, Phys. Rev. E 65 (2002) 041203.

\section{Appendix. D3Q7 and D3Q19 LB models for the convection-diffusion equation}

The D3Q7 and D3Q19 models have the following sets of discrete velocities

$$
\begin{gathered}
\mathbf{e}_{\alpha}= \begin{cases}(0,0), & \begin{array}{l}
\alpha=0, \\
( \pm 1,0,0),(0, \pm 1,0),(0,0, \pm 1),
\end{array}, \begin{array}{l}
\alpha-6, \\
\text { for D3Q7, and }
\end{array} \\
\mathbf{e}_{\alpha}=\left\{\begin{array}{ll}
(0,0,0), & \alpha=0, \\
( \pm 1,0,0),(0, \pm 1,0),(0,0, \pm 1), & \alpha=1-6,
\end{array}\right. \text { for D3Q19. } \\
( \pm 1, \pm 1,0),( \pm 1,0, \pm 1),(0, \pm 1, \pm 1), \alpha=7-18,\end{cases}
\end{gathered}
$$

The equilibrium distribution functions without the higher order velocity terms are defined

as

$$
\begin{aligned}
& g_{\alpha}^{\mathrm{eq}}=\omega_{\alpha} \phi\left(1+a_{\alpha} \frac{\mathbf{e}_{\alpha} \cdot \mathbf{u}}{c_{s}^{2}}\right), \text { for D3Q7, and } \\
& g_{\alpha}^{\mathrm{eq}}=\omega_{\alpha} \phi\left(1+\frac{\mathbf{e}_{\alpha} \cdot \mathbf{u}}{c_{s}^{2}}\right), \text { for D3Q19. }
\end{aligned}
$$

The transformation matrix $\mathbf{M}$ can be found in [20] for D3Q7 and [9] for D3Q19. Thus the equilibria in the moment space are

$$
\begin{aligned}
& \mathbf{m}^{\mathrm{eq}}=\phi\left(1, u_{x}, u_{y}, u_{z}, a, 0,0\right)^{\mathrm{T}}, \text { for D3Q7, and } \\
& \mathbf{m}^{\mathrm{eq}}=\phi\left(1,-11,3, u_{x},-\frac{2}{3} u_{x}, u_{y},-\frac{2}{3} u_{y}, u_{z},-\frac{2}{3} u_{z}, 0,0,0,0,0,0,0,0,0,0\right)^{\mathrm{T}}, \text { for D3Q19. }
\end{aligned}
$$

The weight coefficients in Eq. (A2a) for D3Q7 can then be written as 


$$
a_{\alpha}=\frac{7}{6-a}, \text { and } \omega_{\alpha}=\left\{\begin{array}{l}
(1+a) / 7, \quad \alpha=0, \\
(6-a) / 42, \alpha=1-6,
\end{array}\right. \text { for D3Q7 }
$$

With a particular choice $a=3 / 4$, the coefficients in (A4) become the same as those in [20] and $\varepsilon_{D}$ $=1 / 4$ is noticed for the relationship in Eq. (8). This choice is used in the present work when applying D3Q7. For D3Q19, the weight coefficients in Eq. (A2b) follows those in $[44,8,9]$

$$
\omega_{\alpha}=\left\{\begin{array}{l}
1 / 3, \alpha=0, \\
1 / 18, \alpha=1-6, \quad \text { for D3Q19, } \\
1 / 36, \alpha=7-18,
\end{array}\right.
$$

and $\varepsilon_{D}=1 / 3$ is maintained for the relationship in Eq. (8). 


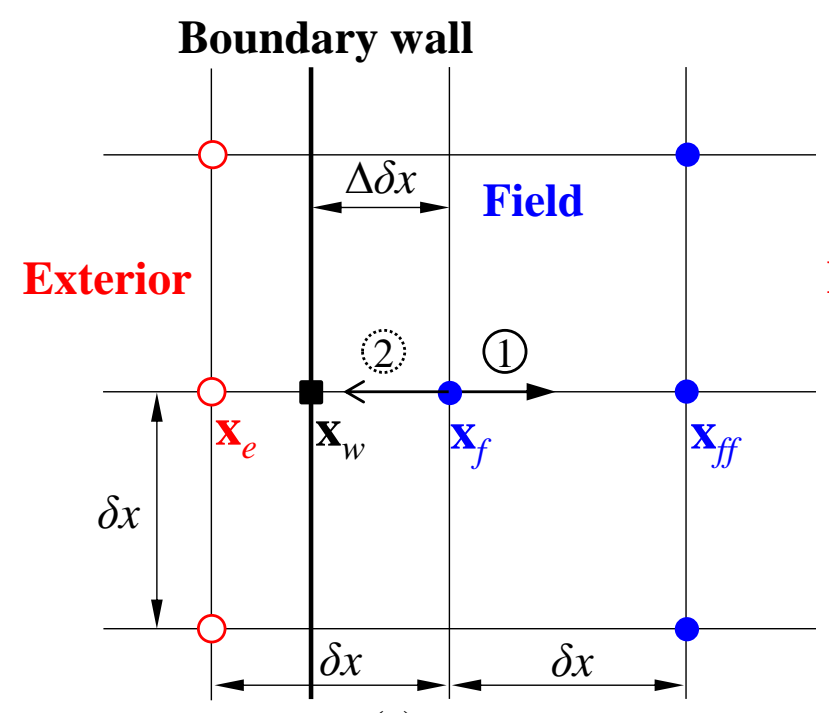

(a)

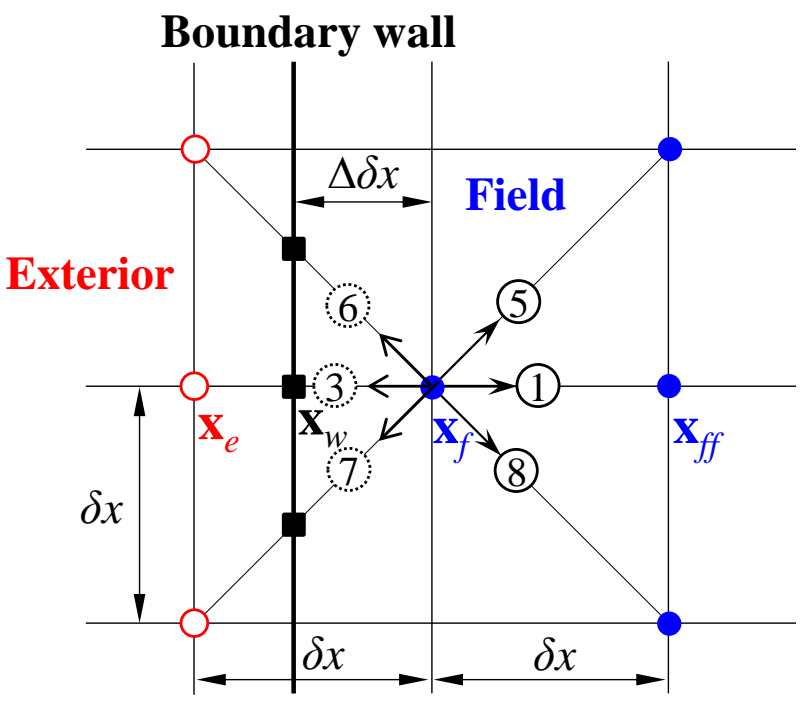

(b)

Fig. 1. Schematic depiction of the lattice links intersected by a boundary wall in (a) D2Q5, and (b) D2Q9 LB models.

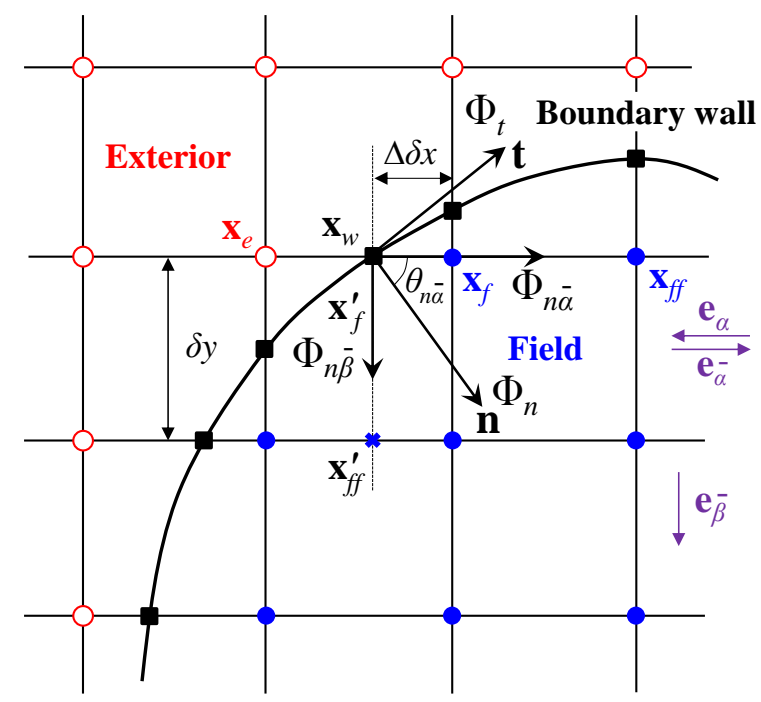

Fig. 2. Layout of the D2Q5 lattice and a curved boundary (solid circles: field nodes; solid squares: boundary nodes; open circles: exterior nodes). Adapted from [24]. 


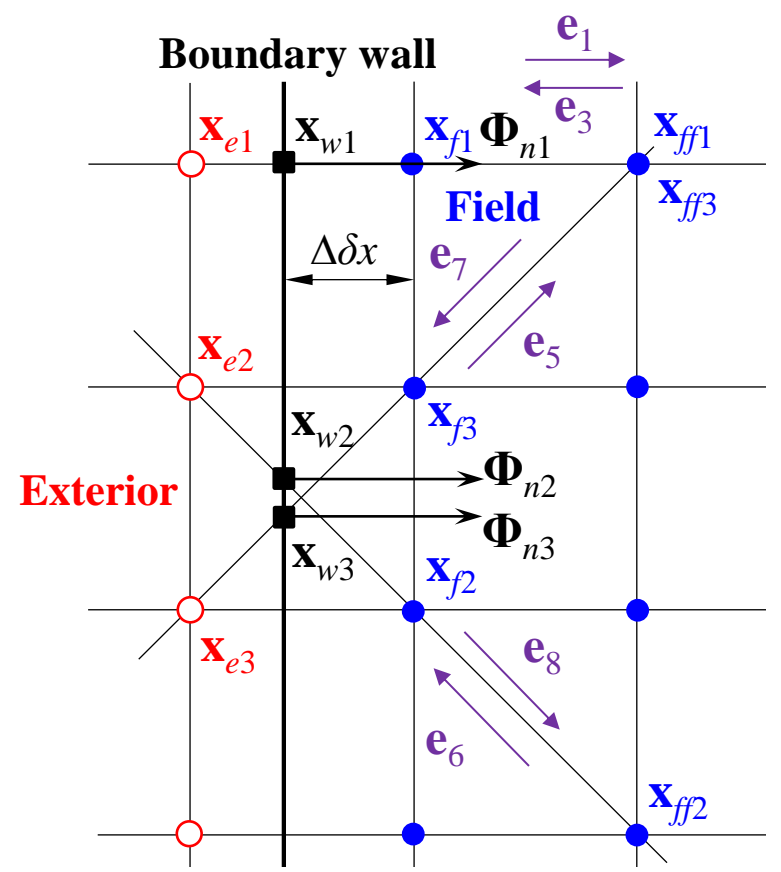

Fig. 3. Layout of the D2Q9 lattice and a straight boundary for the illustration of boundary flux evaluation.

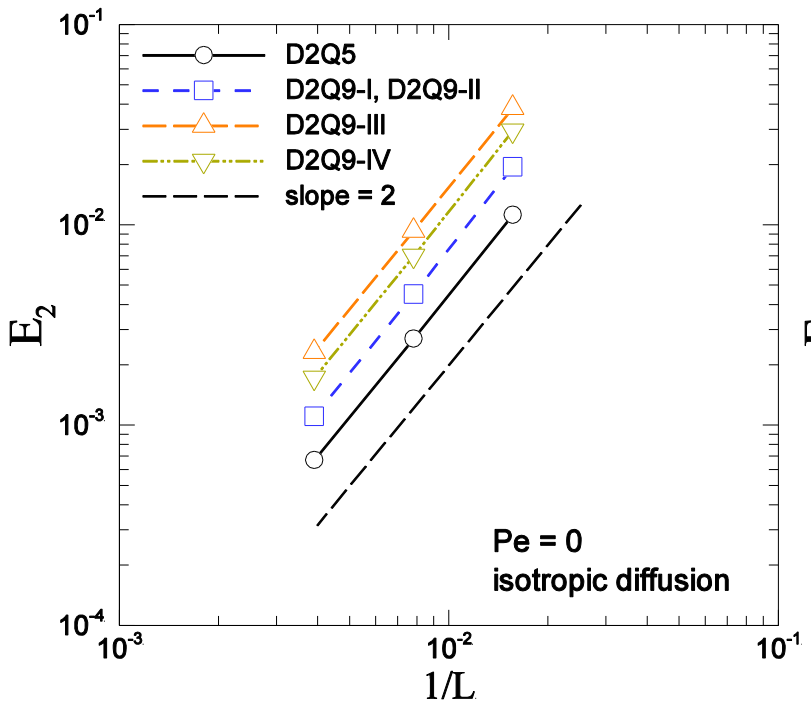

(a)

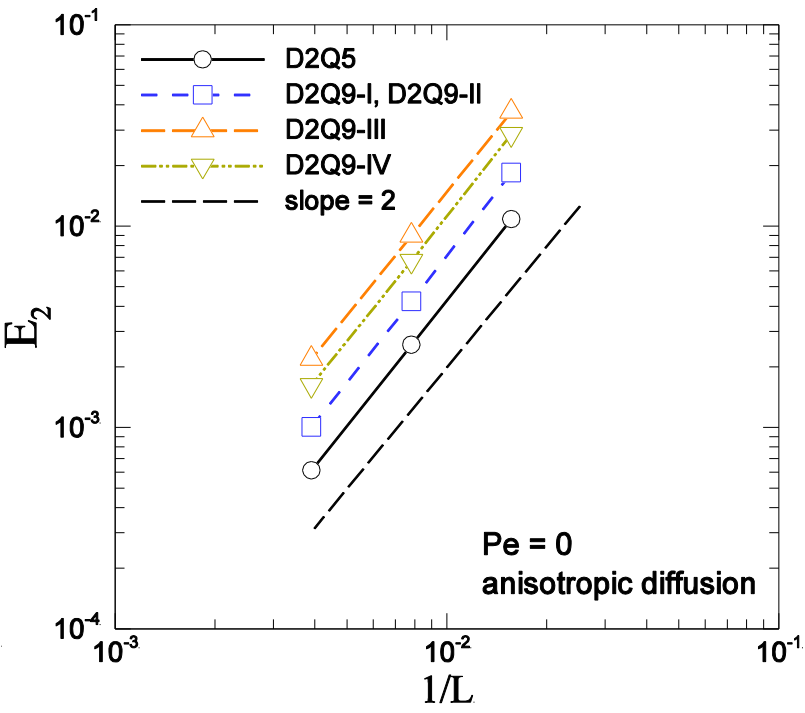

(b) 


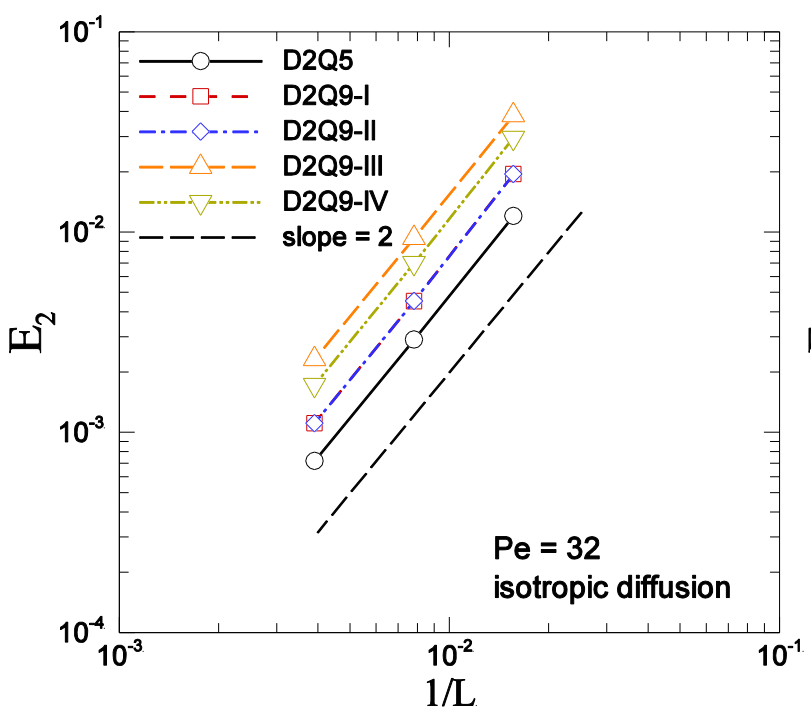

(c)

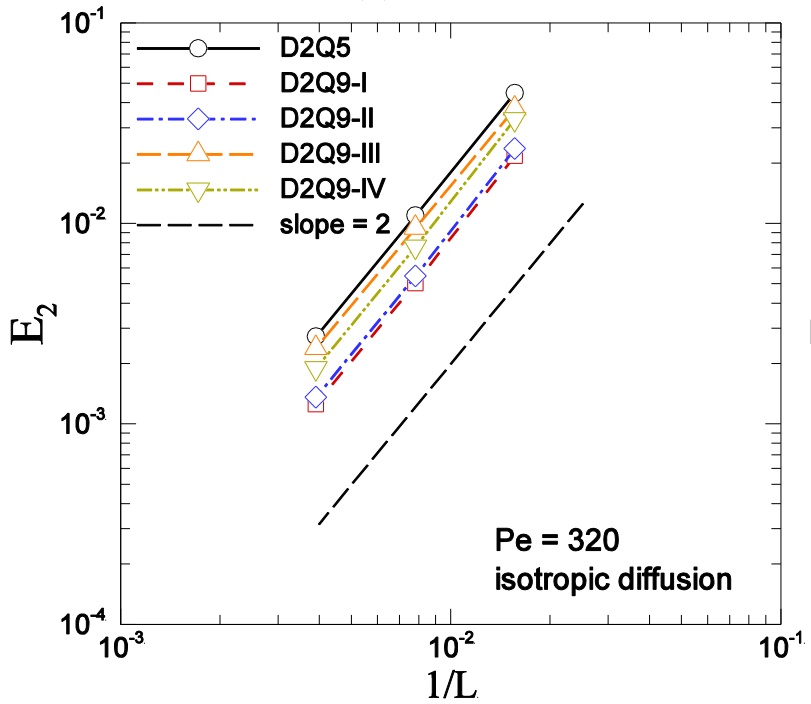

(e)

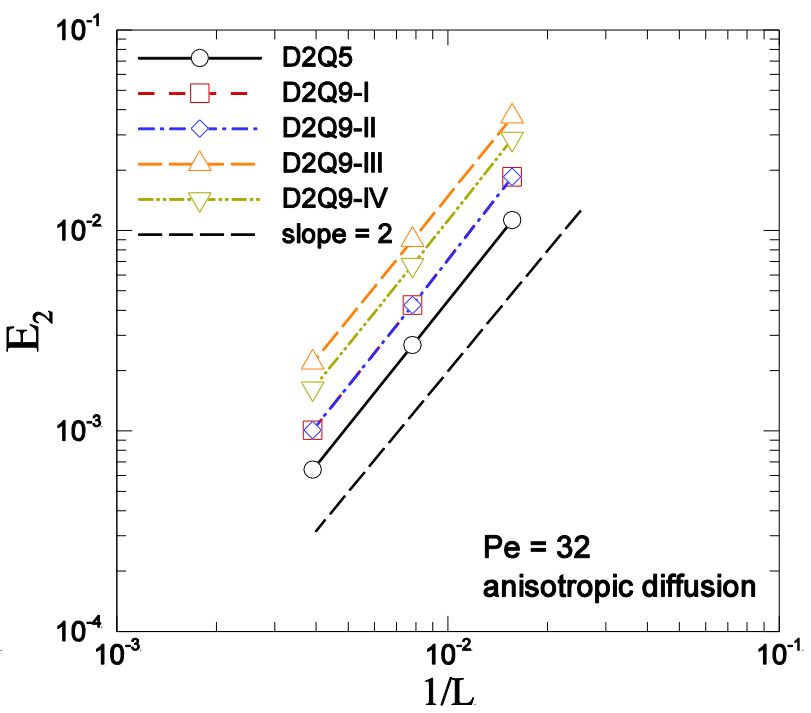

(d)

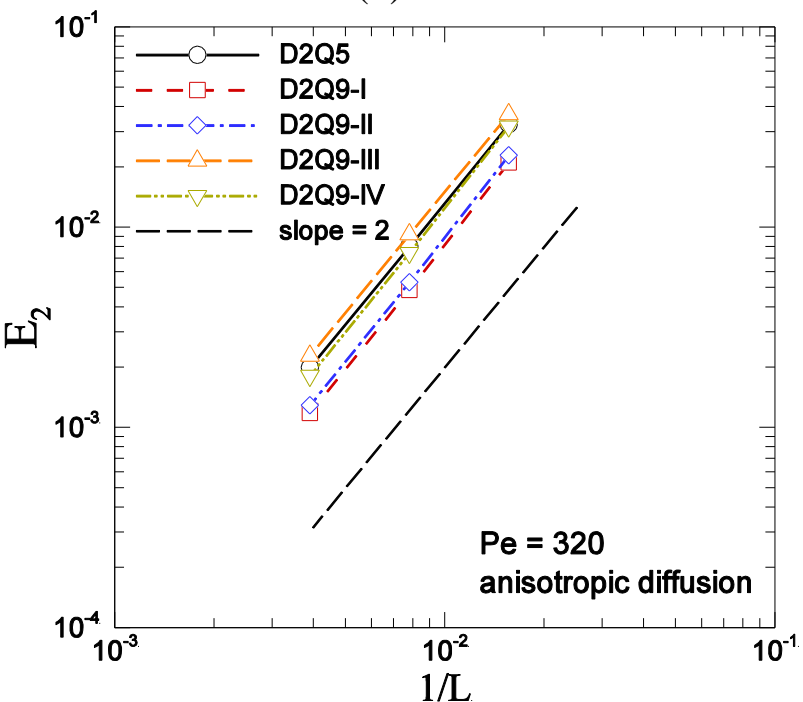

(f)

Fig. 4. Relative $L_{2}$-norm error, $E_{2}$, of the interior distribution of $\phi$ versus the grid resolution, $1 / L$, for (a) isotropic diffusion at $P e=0$, (b) anisotropic diffusion at $P e=0$, (c) isotropic diffusion at $P e=32$, (d) anisotropic diffusion at $P e=32$, (e) isotropic diffusion at $P e=320$, and (f) anisotropic diffusion at $P e=320$ in the Gaussian hill convection-diffusion problem. 


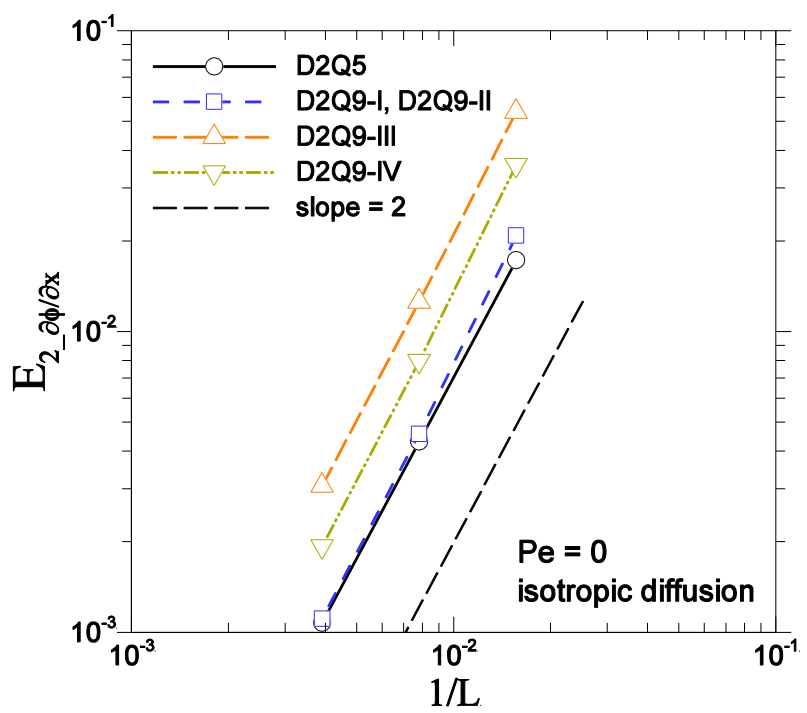

(a)

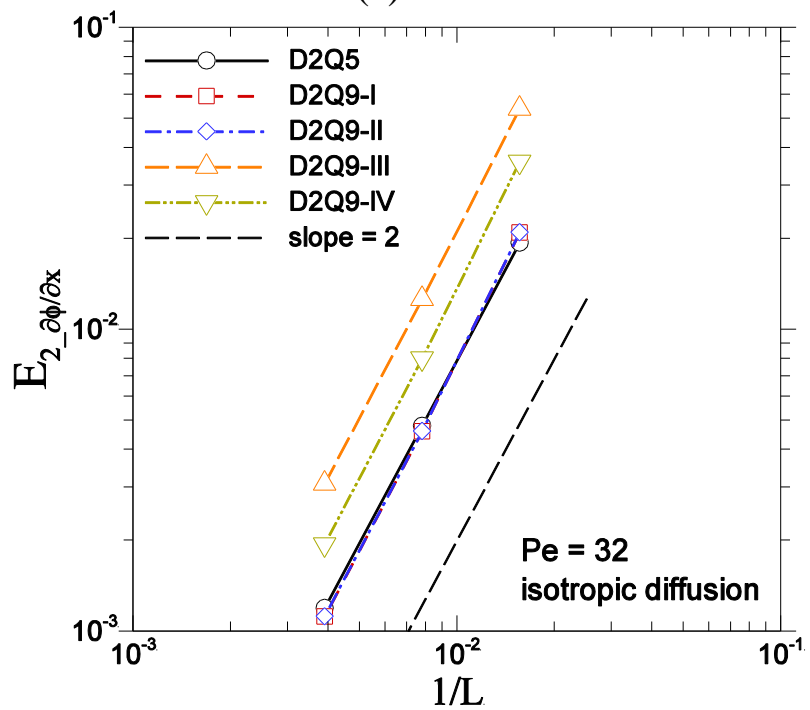

(c)

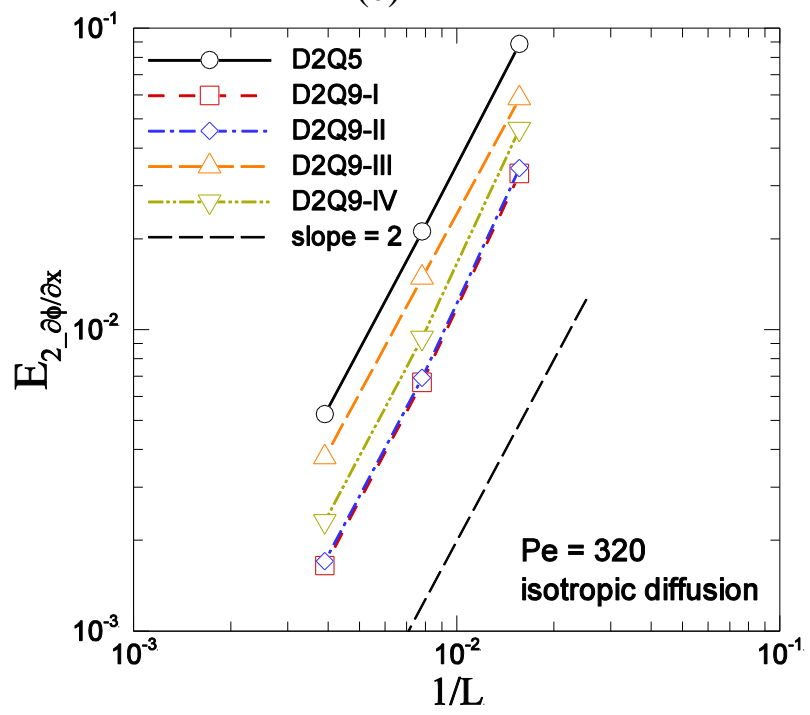

(e)

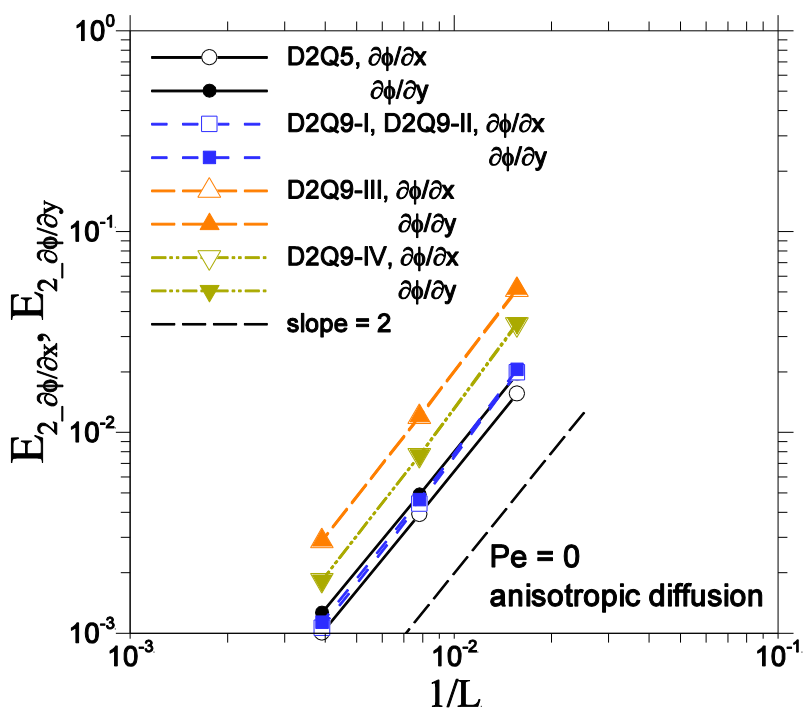

(b)

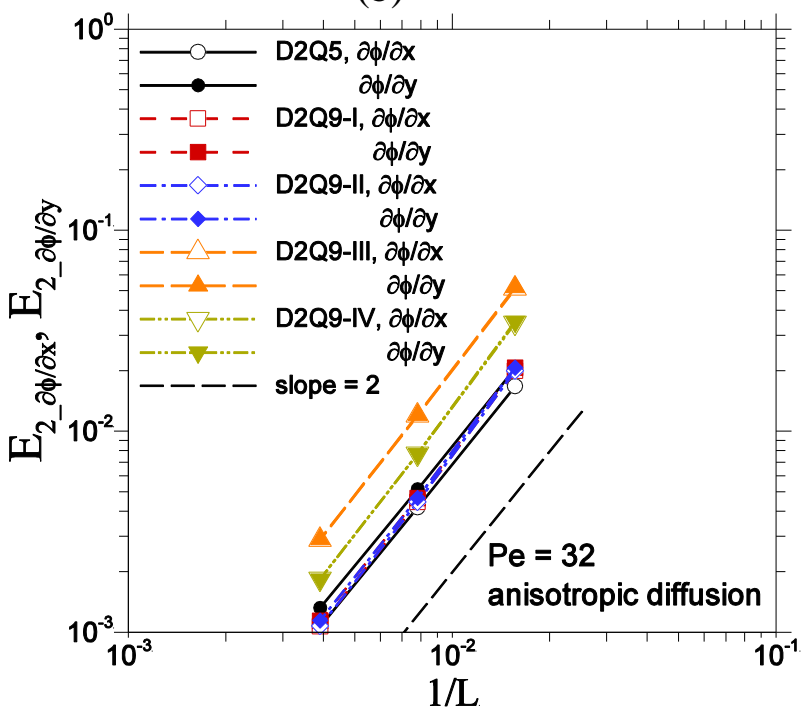

(d)

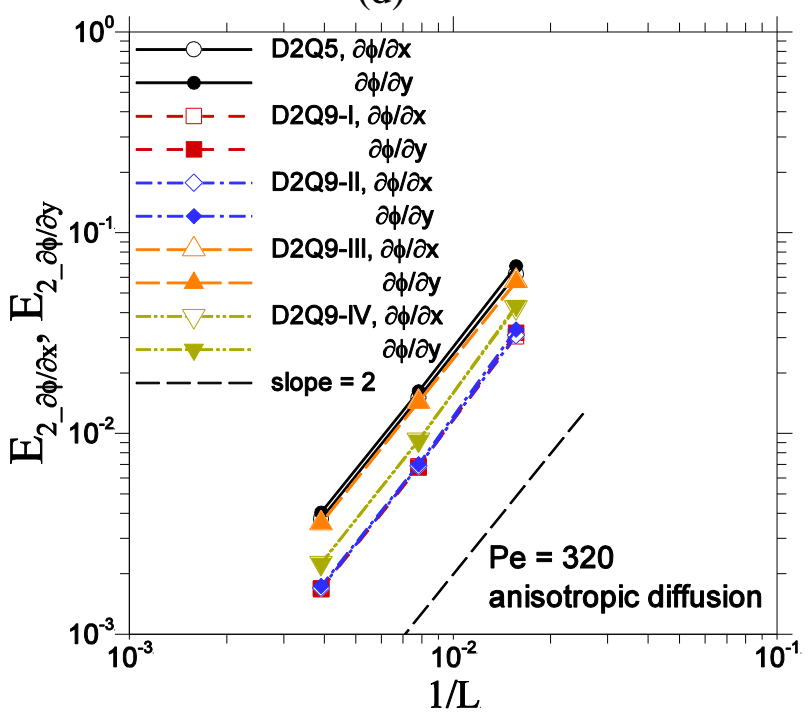

(f) 
Fig. 5. Relative $L_{2}$-norm errors, $E_{2} \partial \phi / \partial x$ and $E_{2} \partial \phi / \partial y$, of the interior derivatives versus the grid resolution, $1 / L$, for (a) isotropic diffusion at $P e=0$, (b) anisotropic diffusion at $P e=0$, (c) isotropic diffusion at $P e=32$, (d) anisotropic diffusion at $P e=32$, (e) isotropic diffusion at $P e=$ 320 , and (f) anisotropic diffusion at $P e=320$ in the Gaussian hill convection-diffusion problem.

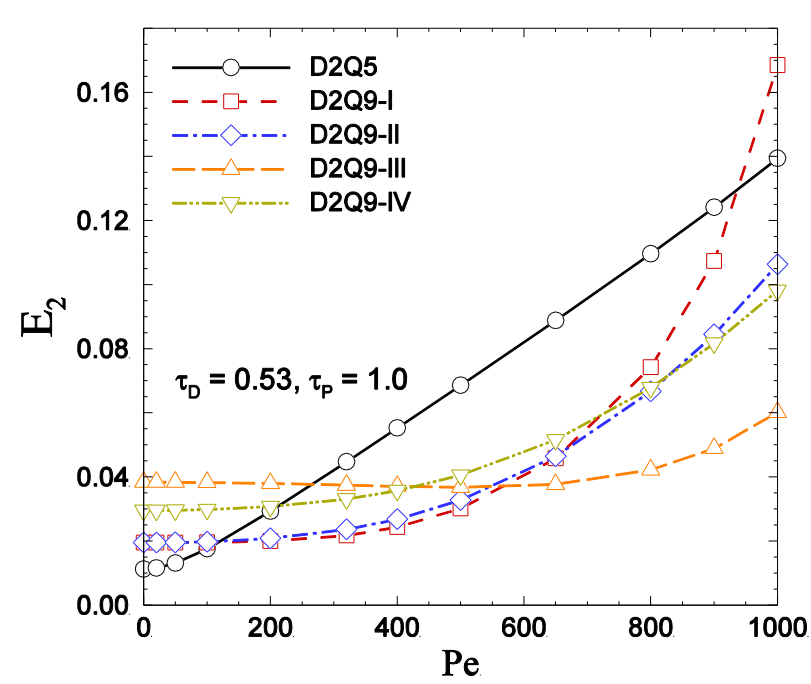

(a)

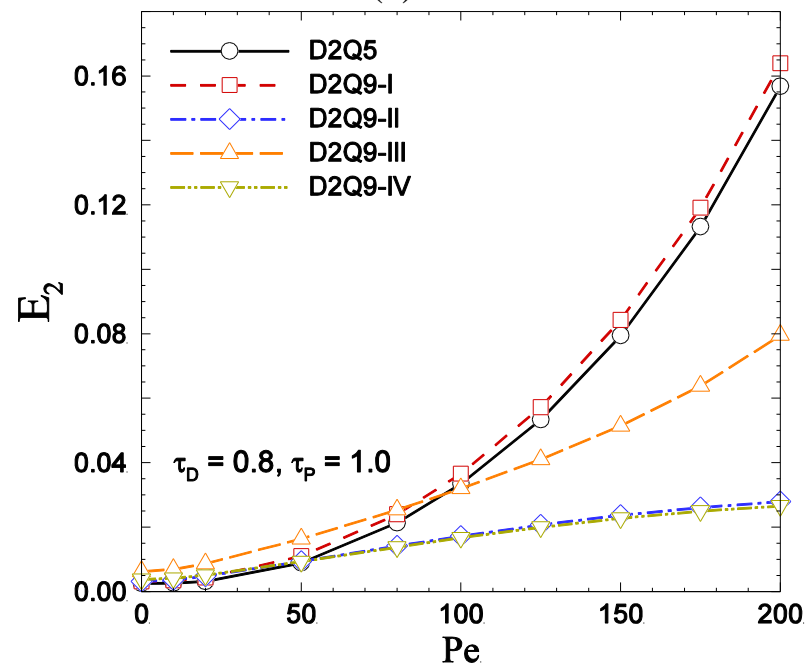

(c)

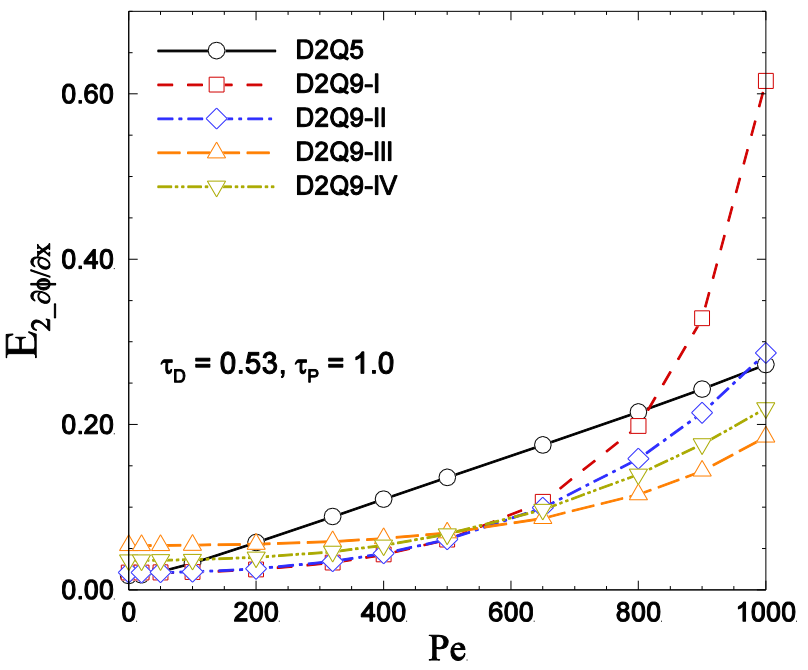

(b)

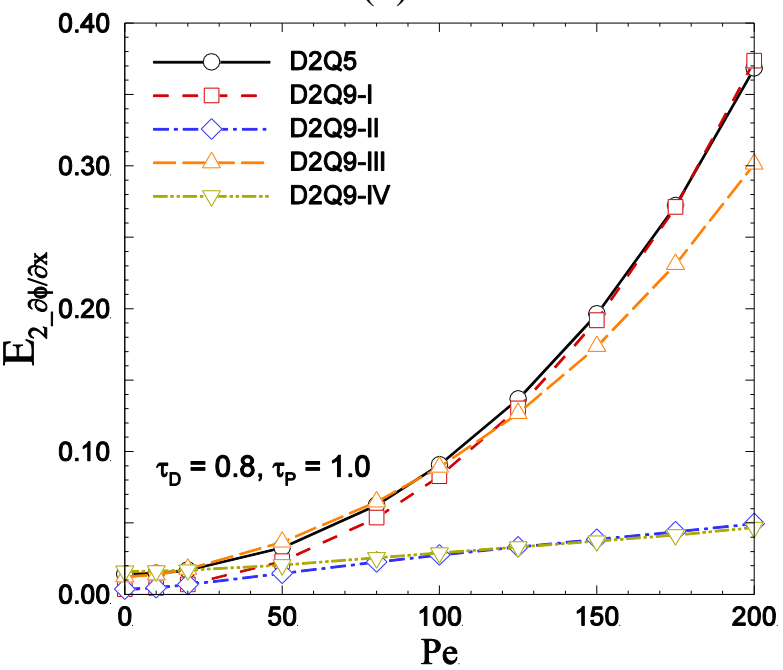

(d)

Fig. 6. Relative $L_{2}$-norm errors (a) $E_{2}$ at $\left(\tau_{D}, \tau_{P}\right)=(0.53,1.0)$, (b) $E_{2} \partial \phi / \partial x$ at $\left(\tau_{D}, \tau_{P}\right)=(0.53,1.0)$, (c) $E_{2}$ at $\left(\tau_{D}, \tau_{P}\right)=(0.8,1.0)$, and (d) $E_{2 \_} \_\phi / \partial x$ at $\left(\tau_{D}, \tau_{P}\right)=(0.8,1.0)$ versus the Péclet number, $P e$, for the Gaussian hill convection-diffusion problem. 


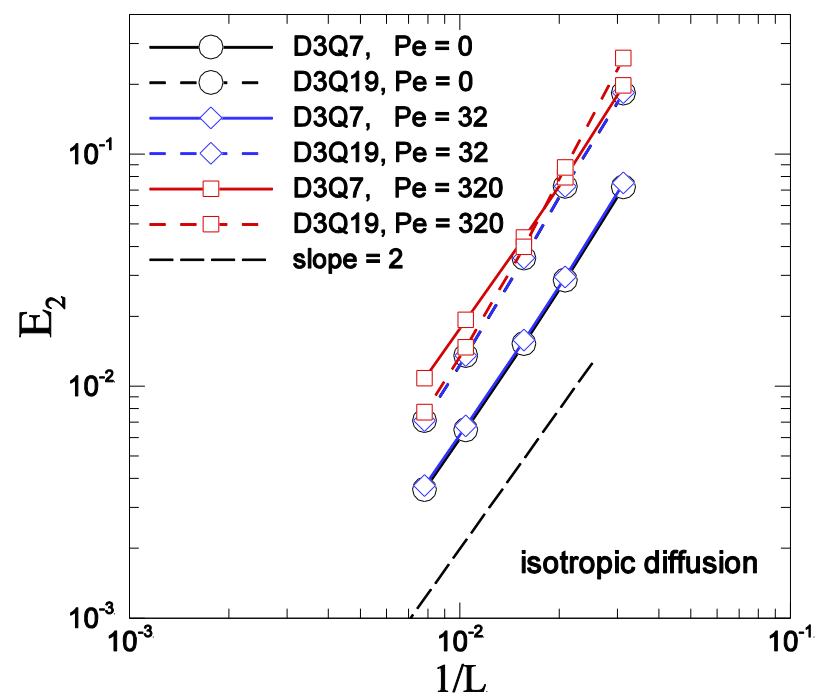

(a)

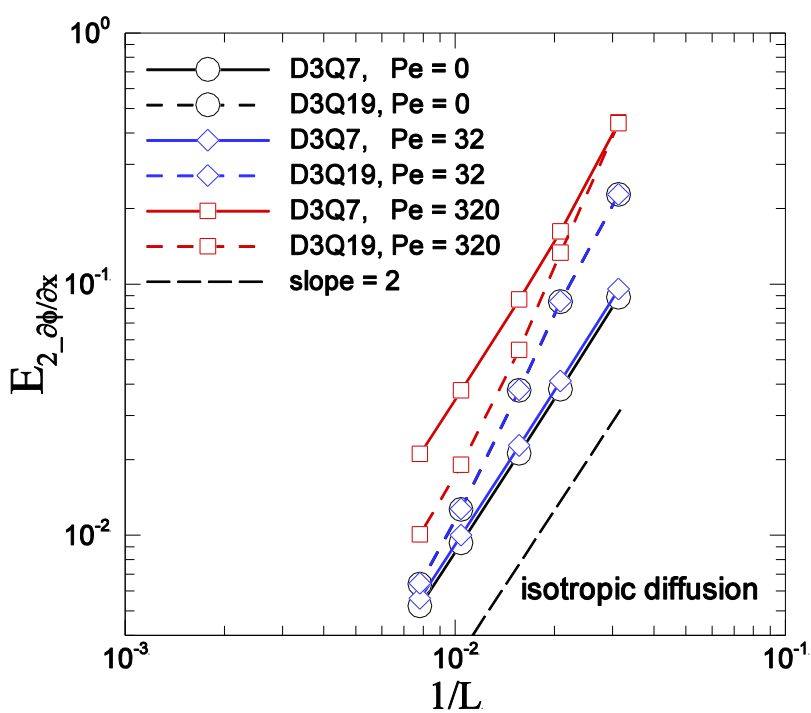

(b)

Fig. 7. Relative $L_{2}$-norm errors (a) $E_{2}$, and (b) $E_{2 \_} \partial \phi / \partial x$ versus the grid resolution, $1 / L$, for the Gaussian hill convection-diffusion problem in 3D.

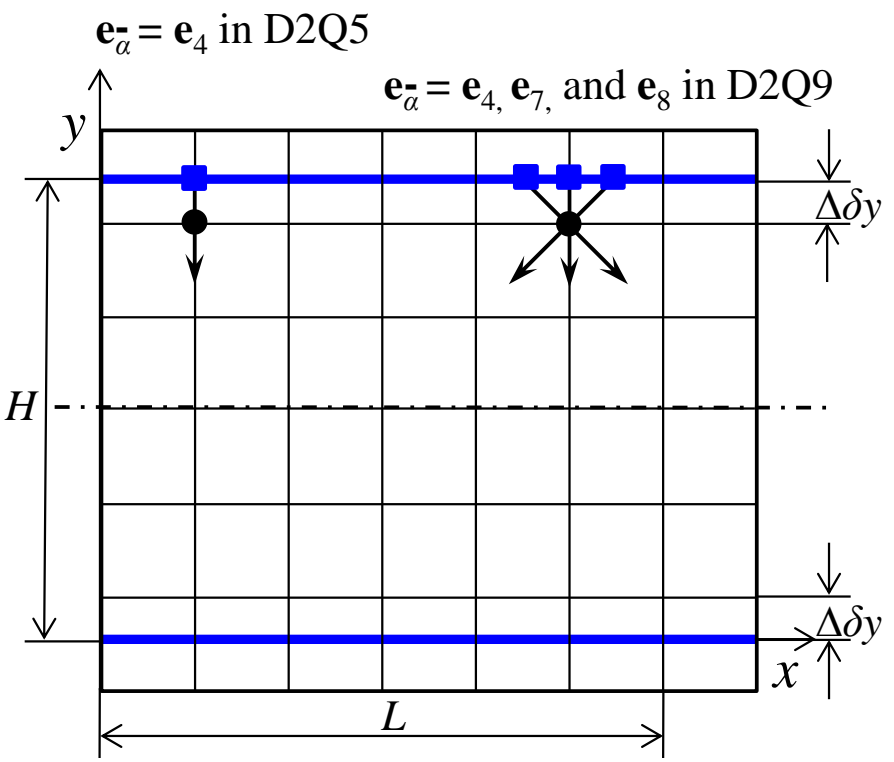

Fig. 8. Schematic depiction of the computational domain and the lattice distribution for convection-diffusion in a channel. 


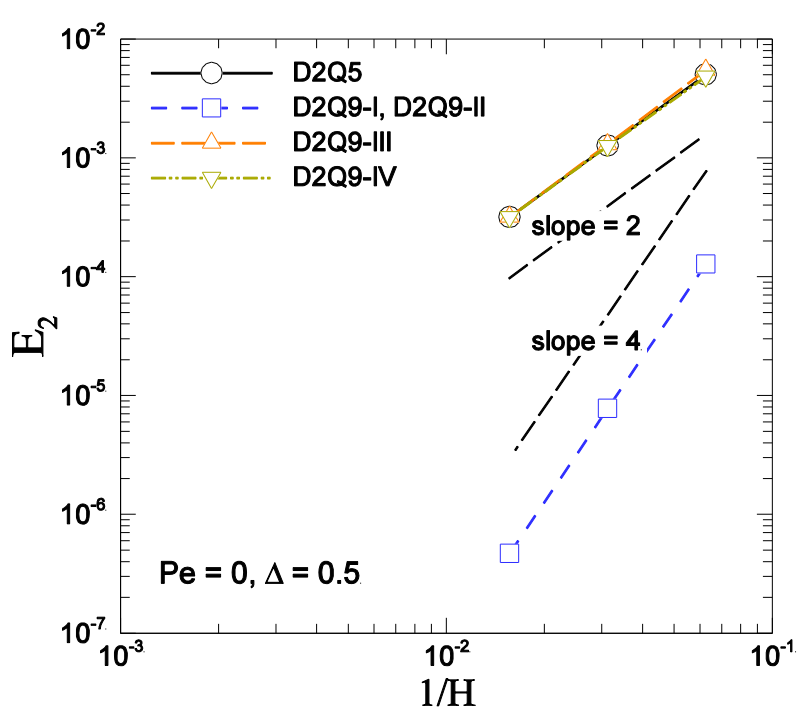

(a)

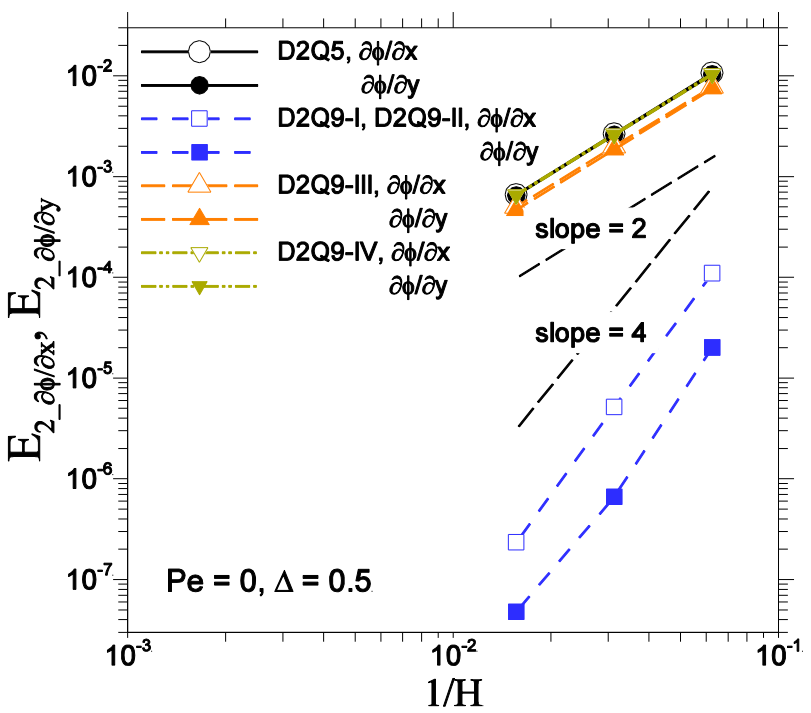

(b)

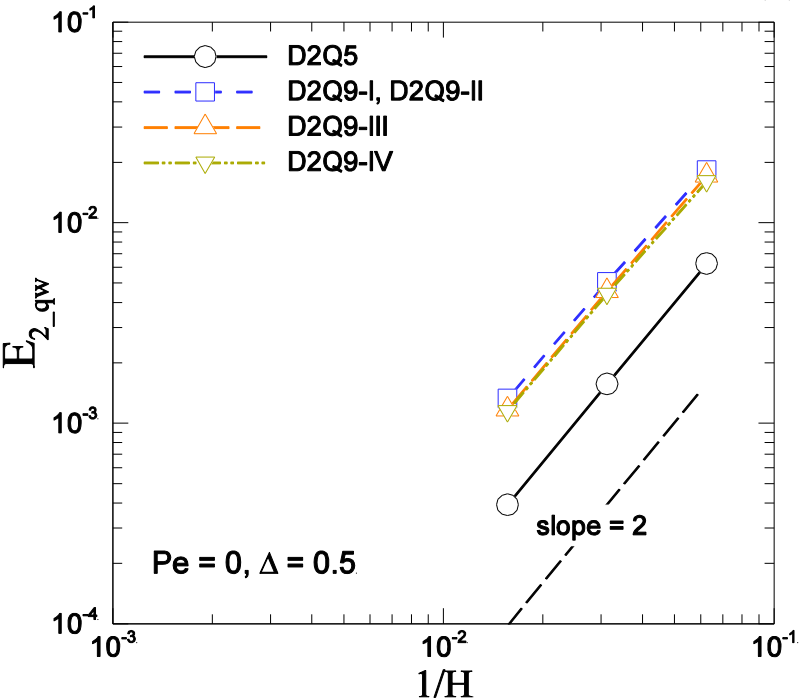

(c)

Fig. 9. Relative $L_{2}$-norm errors, (a) $E_{2}$, (b) $E_{2} \partial \phi / \partial x$ and $E_{2} \partial \phi / \partial y$, and (c) $E_{2 \_q w}$ versus the grid resolution, $1 / H$, for the steady convection-diffusion in a $2 \mathrm{D}$ channel with Dirichlet conditions at $P e=0$ and $\Delta=0.5$. 


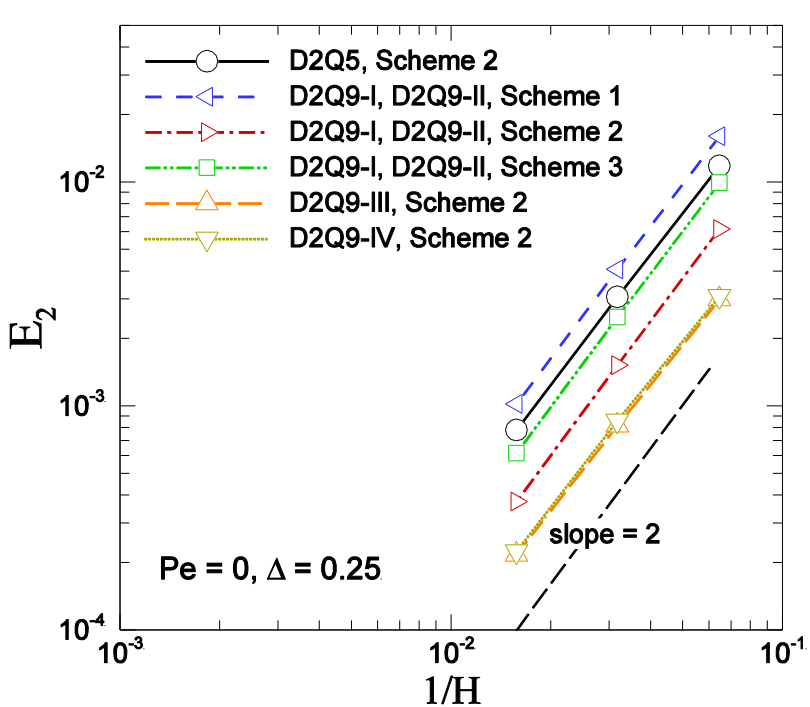

(a)

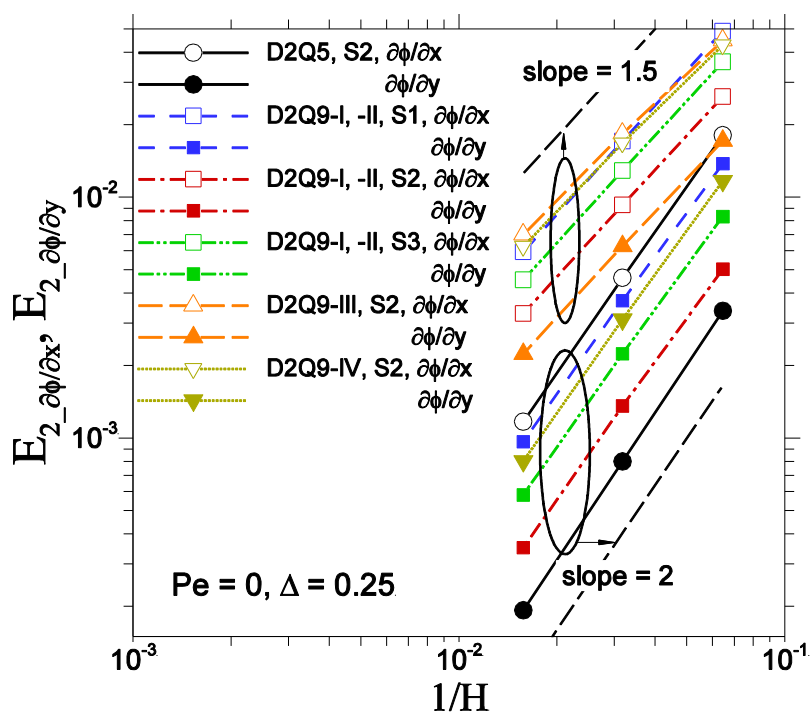

(b)

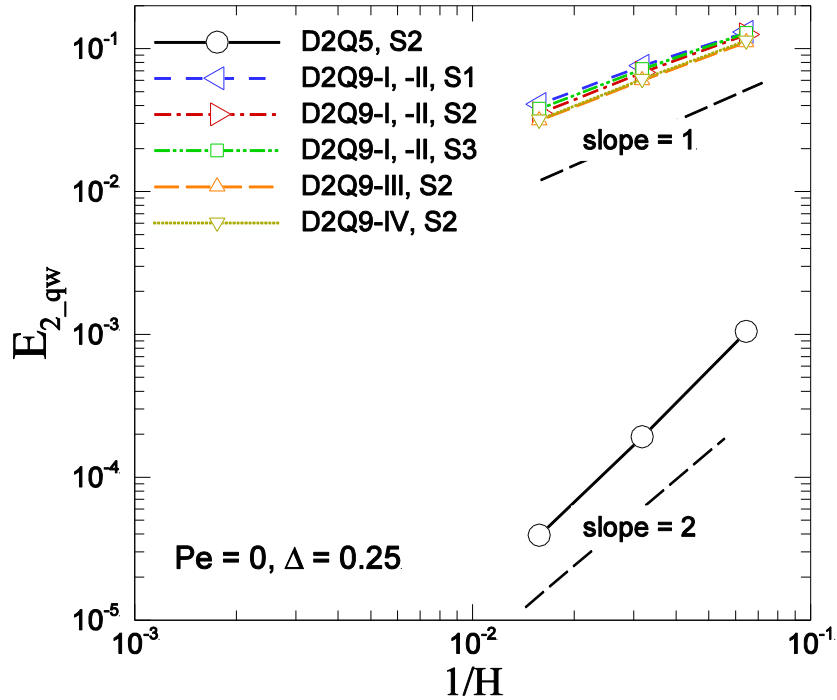

(c)

Fig. 10. Relative $L_{2}$-norm errors, (a) $E_{2}$, (b) $E_{2 \_} \partial \phi / \partial x$ and $E_{2 \_} \partial \phi / \partial y$, and (c) $E_{2 \_q w}$ versus the grid resolution, $1 / H$, for the steady convection-diffusion in a $2 \mathrm{D}$ channel with Dirichlet conditions at $P e=0$ and $\Delta=0.25$. 


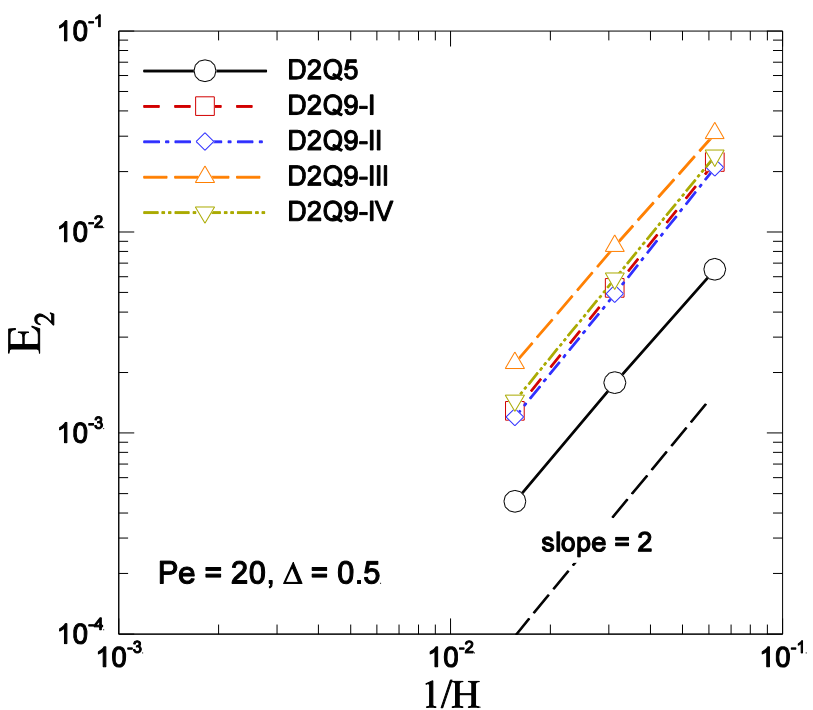

(a)

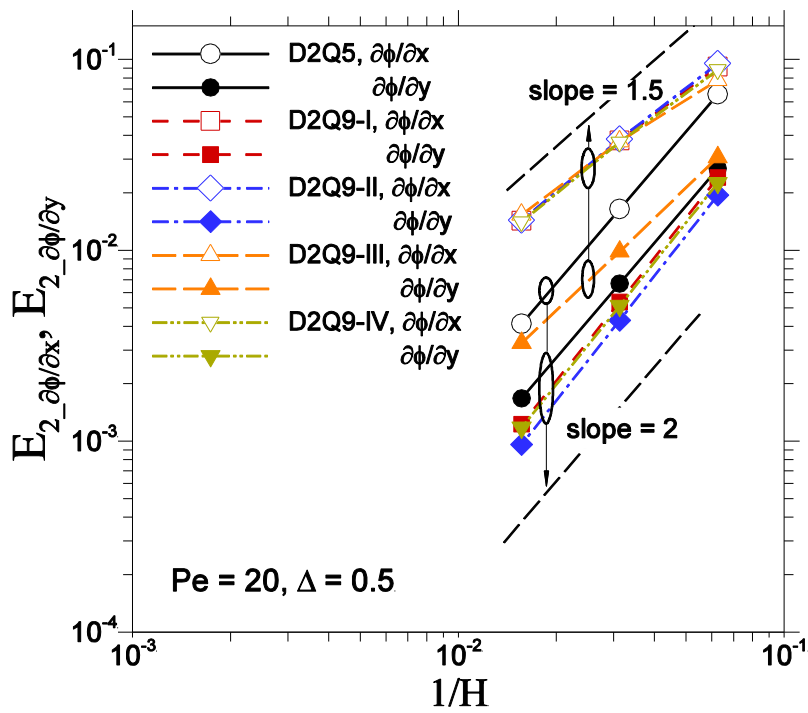

(b)

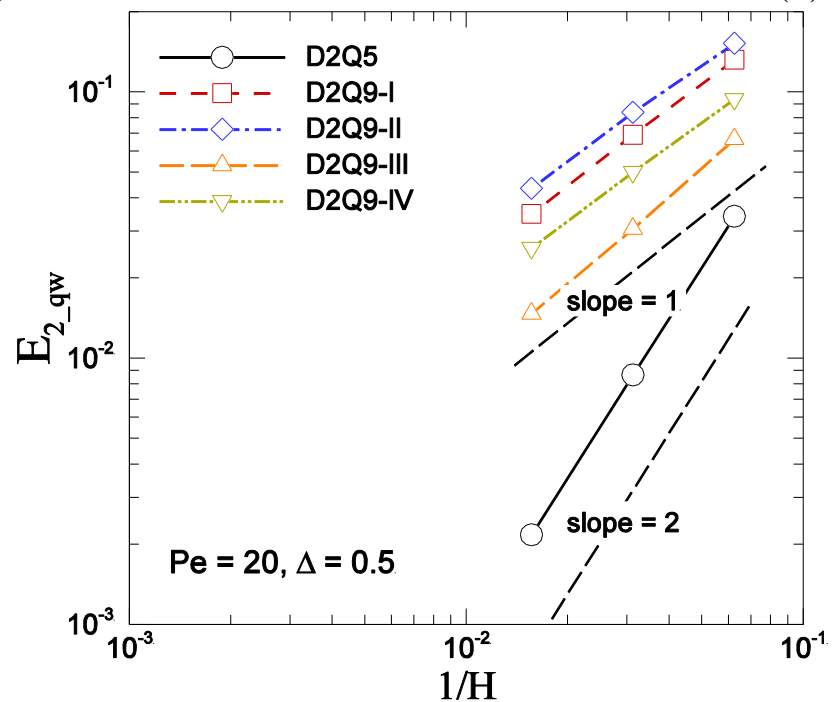

(c)

Fig. 11. Relative $L_{2}$-norm errors, (a) $E_{2}$, (b) $E_{2} \partial \phi / \partial x$ and $E_{2} \partial \phi / \partial y$, and (c) $E_{2 \_q w}$ versus the grid resolution, $1 / H$, for the steady convection-diffusion in a $2 \mathrm{D}$ channel with Dirichlet conditions at $P e=20$ and $\Delta=0.5$. 


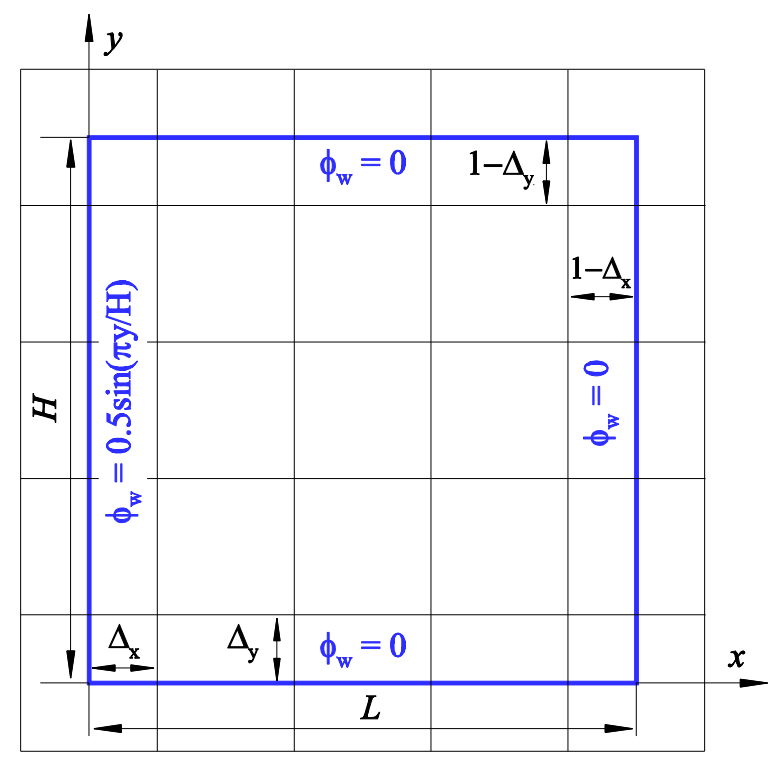

Fig. 12. Schematic depiction of the computational domain and lattice distribution for the square block diffusion problem with Dirichlet conditions. 


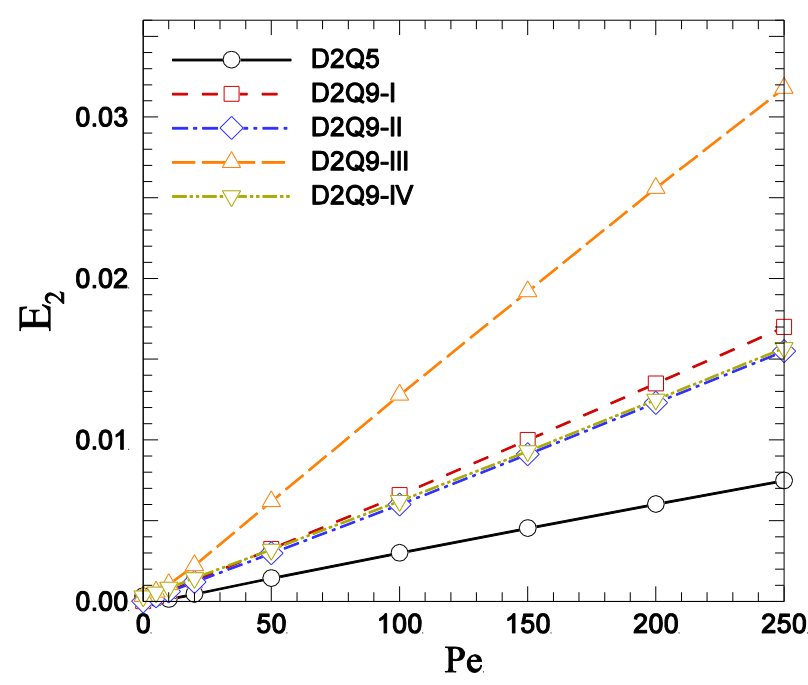

(a)

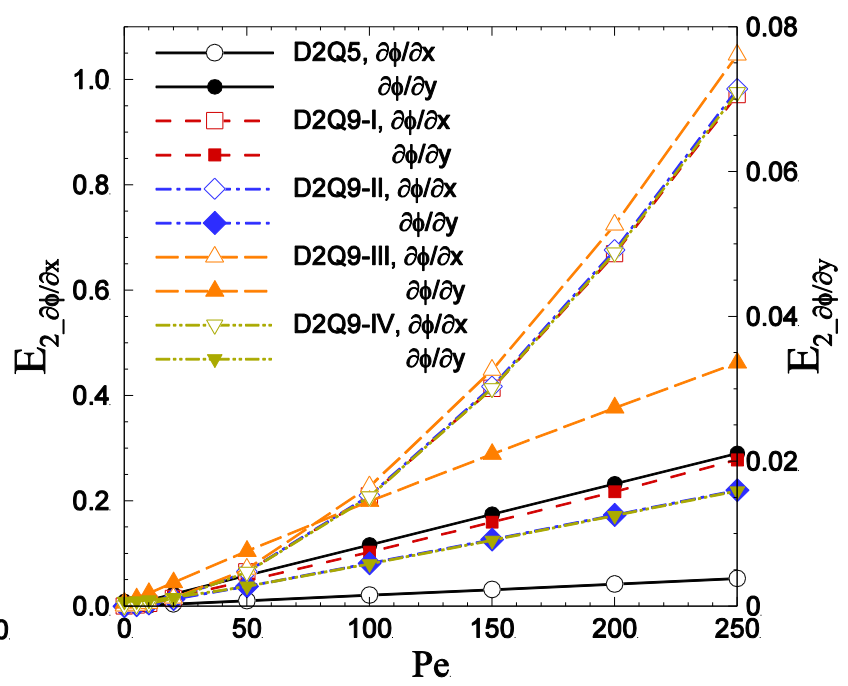

(b)

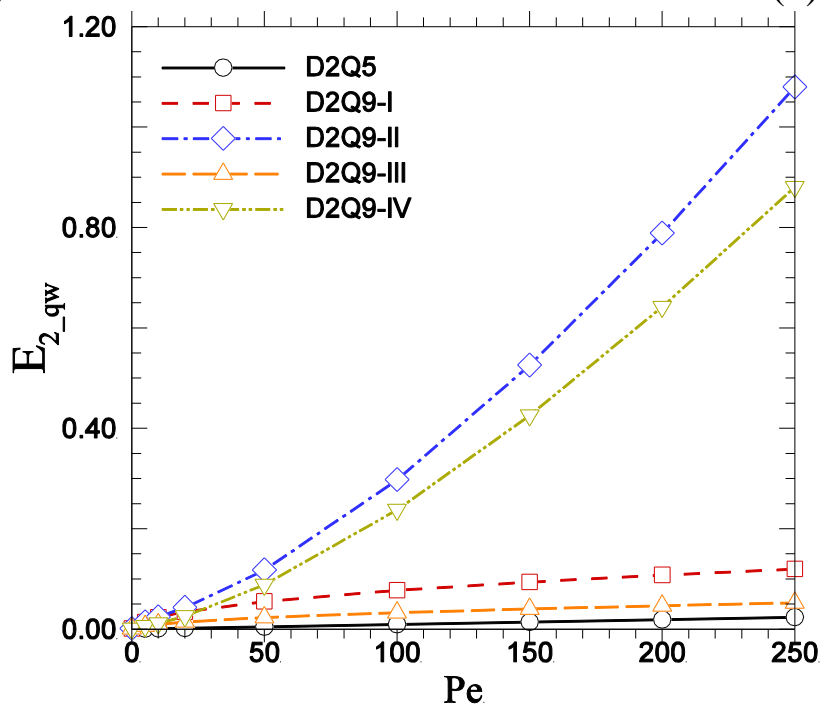

(c)

Fig. 13. Relative $L_{2}$-norm errors, (a) $E_{2}$, (b) $E_{2 \_} \partial \phi / \partial x$ and $E_{2 \_} \partial \phi / \partial y$, and (c) $E_{2 \_q w}$ versus the Péclet number, $P e$, for the steady convection-diffusion in a $2 \mathrm{D}$ channel with Dirichlet conditions. 


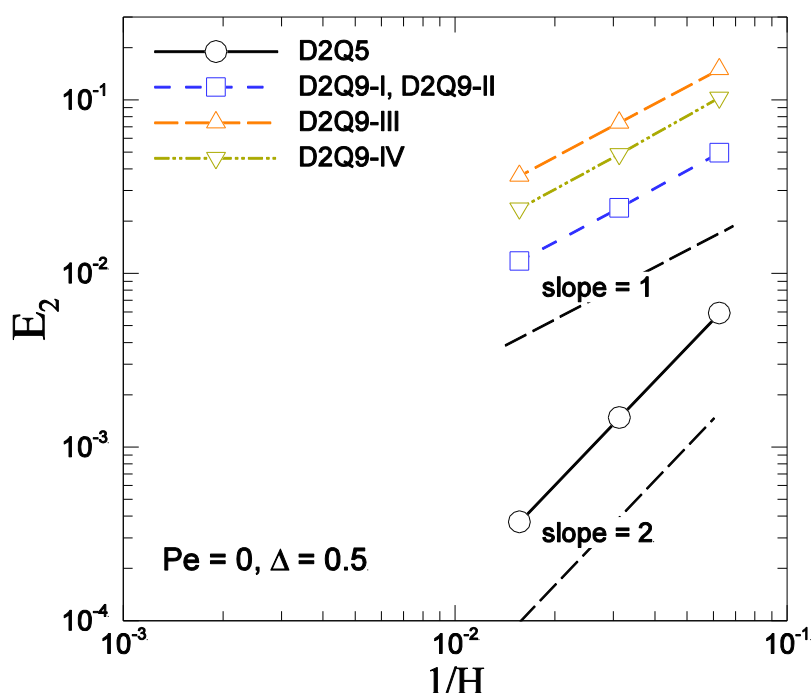

(a)

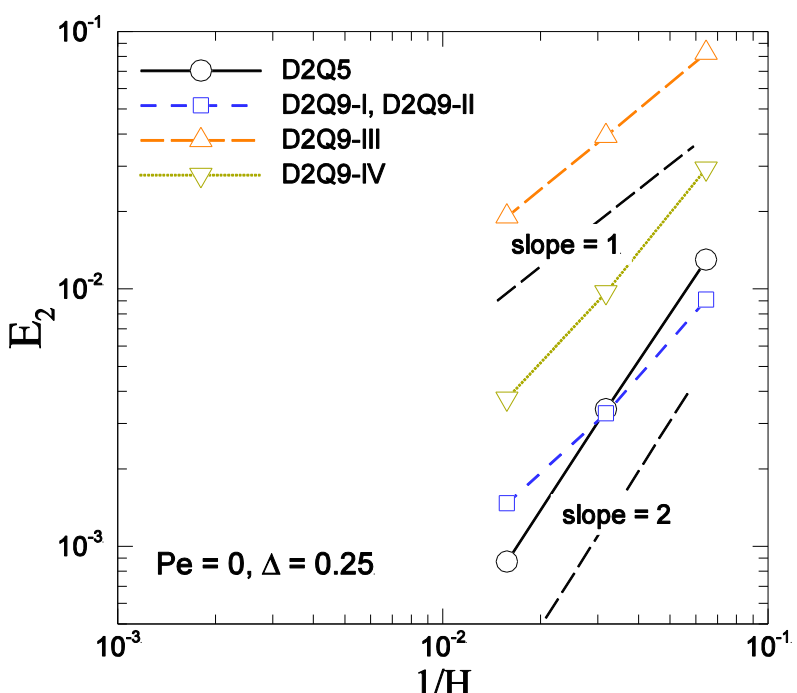

(b)

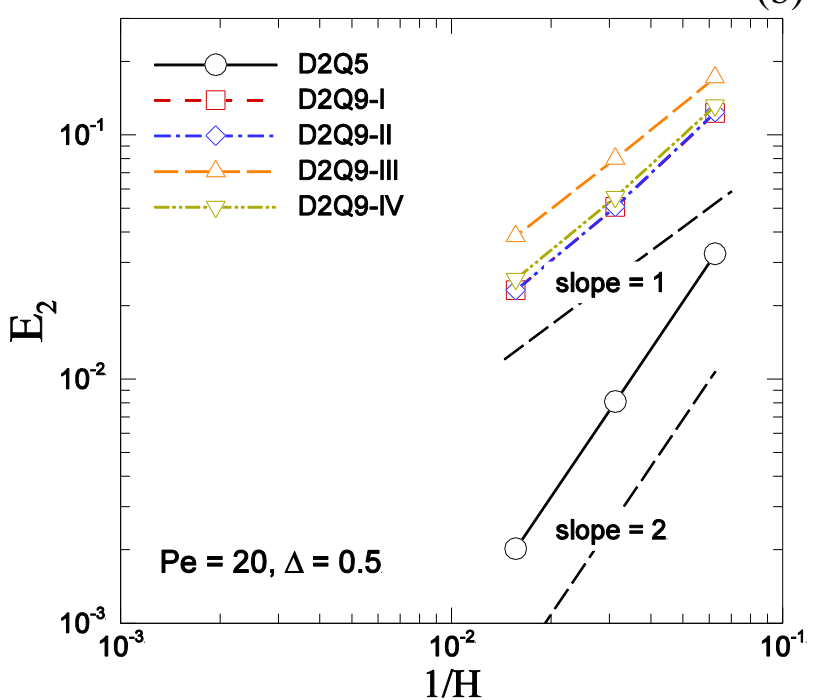

(c)

Fig. 14. Relative $L_{2}$-norm error $E_{2}$, for the interior distribution of $\phi$ versus the grid resolution, $1 / H$, for the steady convection-diffusion in a $2 \mathrm{D}$ channel with Neumann conditions at (a) $P e=0$ and $\Delta=0.5$, (b) $P e=0$ and $\Delta=0.25$, and (c) $P e=20$ and $\Delta=0.5$. 


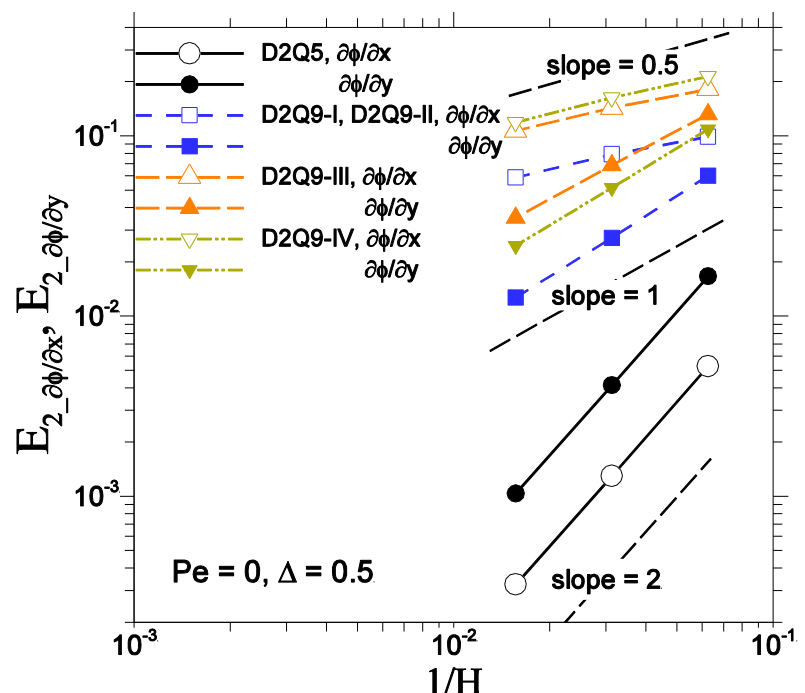

(a)

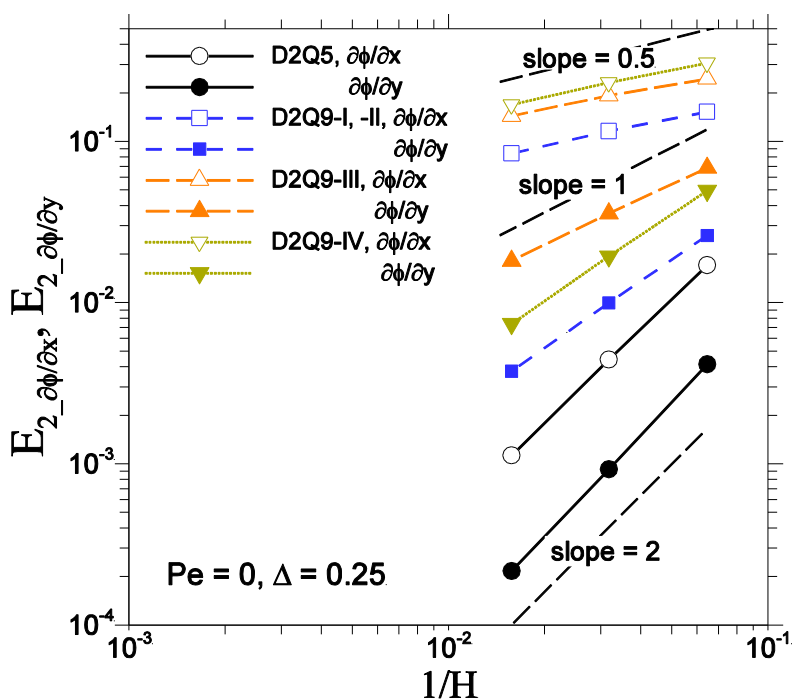

(b)

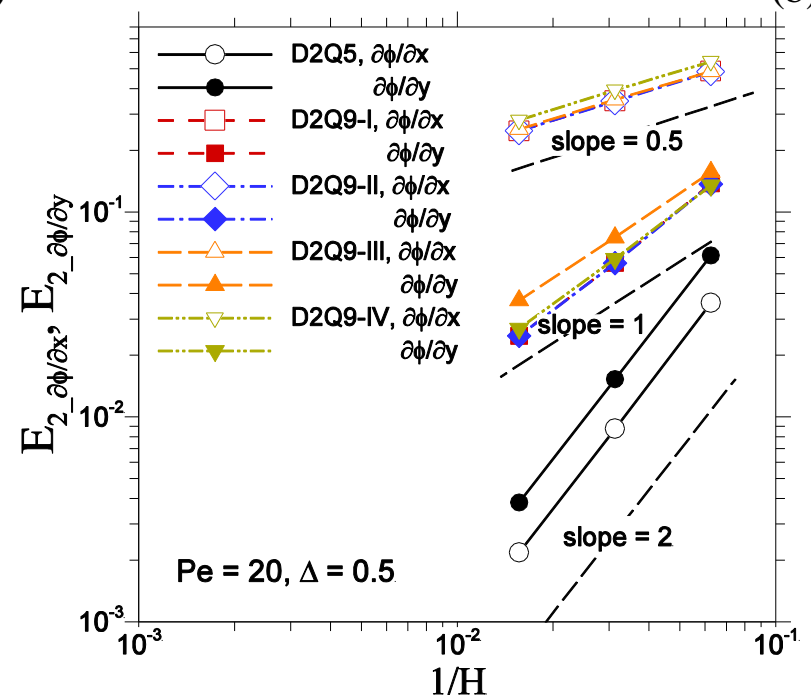

(c)

Fig. 15. Relative $L_{2}$-norm errors, $E_{2 \_} \partial \phi / \partial x$ and $E_{2 \_} \partial \phi / \partial y$, for the interior derivatives of $\phi$ versus the grid resolution, $1 / \mathrm{H}$, for the steady convection-diffusion in a $2 \mathrm{D}$ channel with Neumann conditions at (a) $P e=0$ and $\Delta=0.5$, (b) $P e=0$ and $\Delta=0.25$, and (c) $P e=20$ and $\Delta=0.5$. 


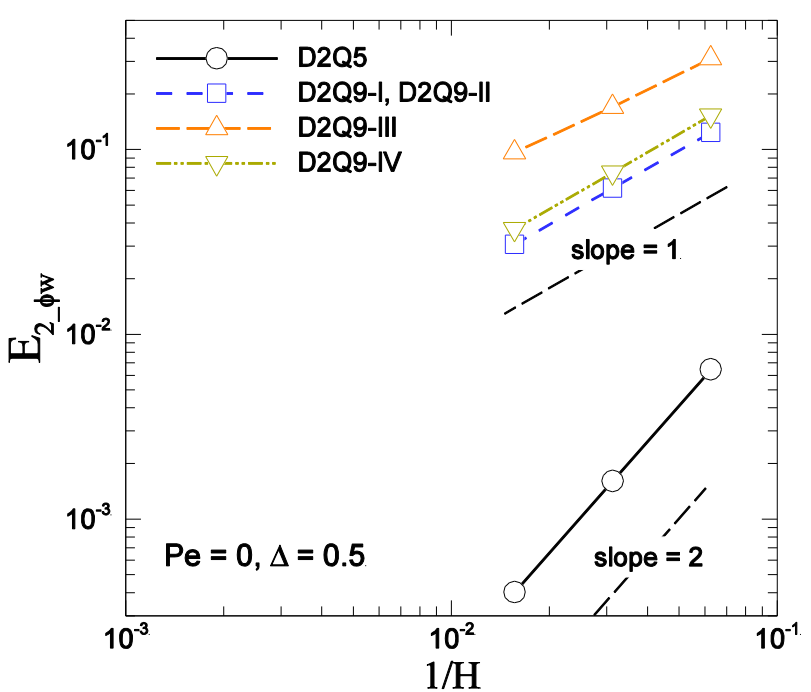

(a)

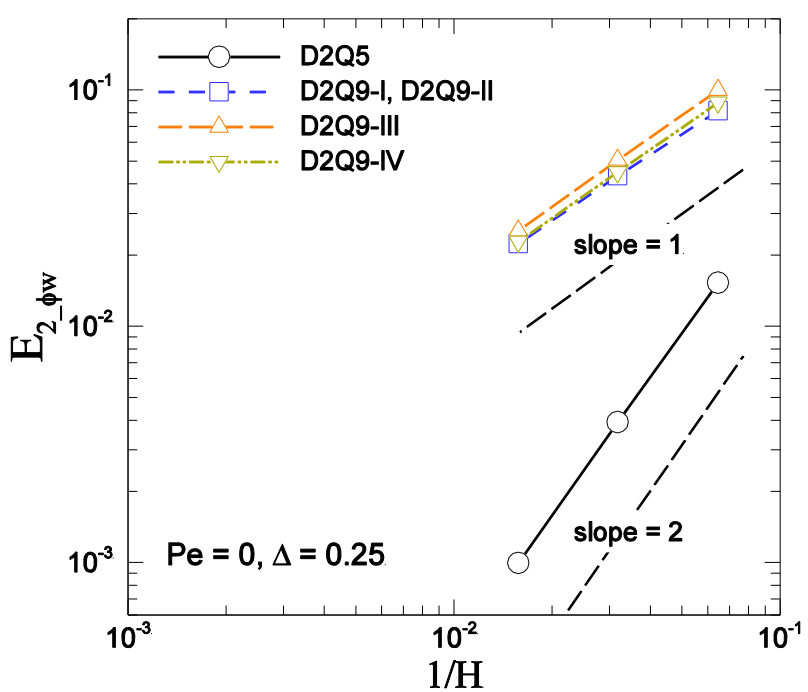

(b)

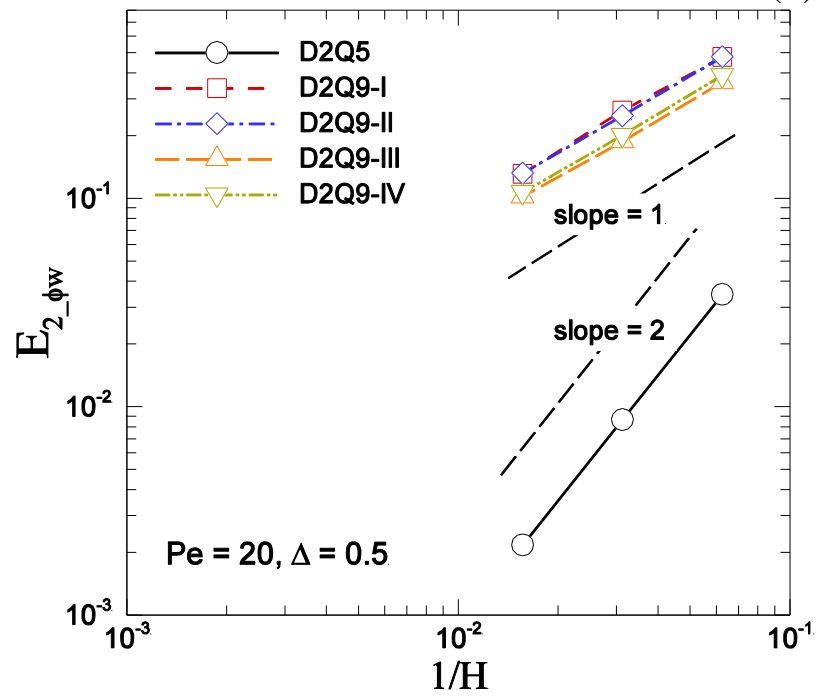

(c)

Fig. 16. Relative $L_{2}$-norm error, $E_{2} \phi_{w}$, for the boundary $\phi_{w}$ values versus the grid resolution, $1 / H$, for the steady convection-diffusion in a $2 \mathrm{D}$ channel with Neumann conditions at (a) $P e=0$ and $\Delta=0.5$, (b) $P e=0$ and $\Delta=0.25$, and (c) $P e=20$ and $\Delta=0.5$. 


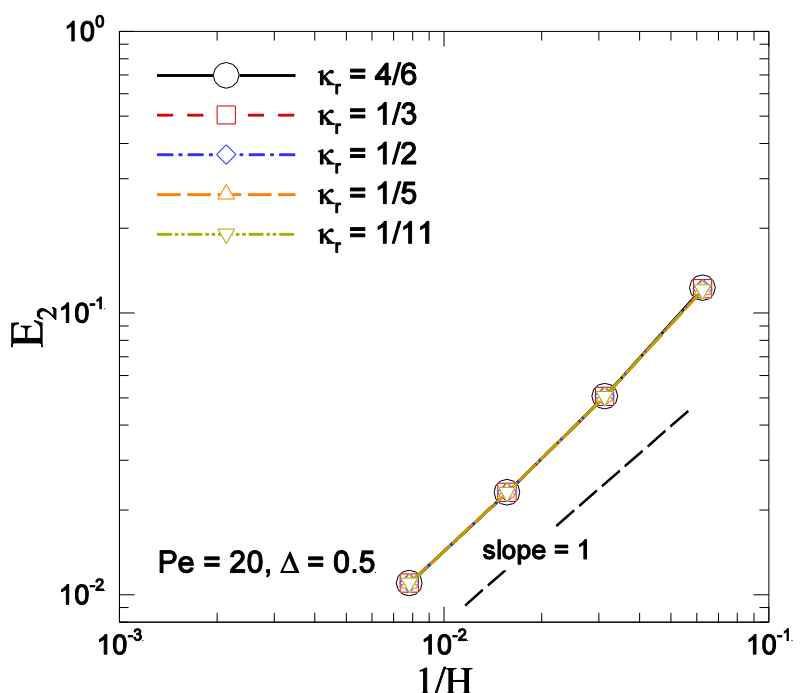

(a)

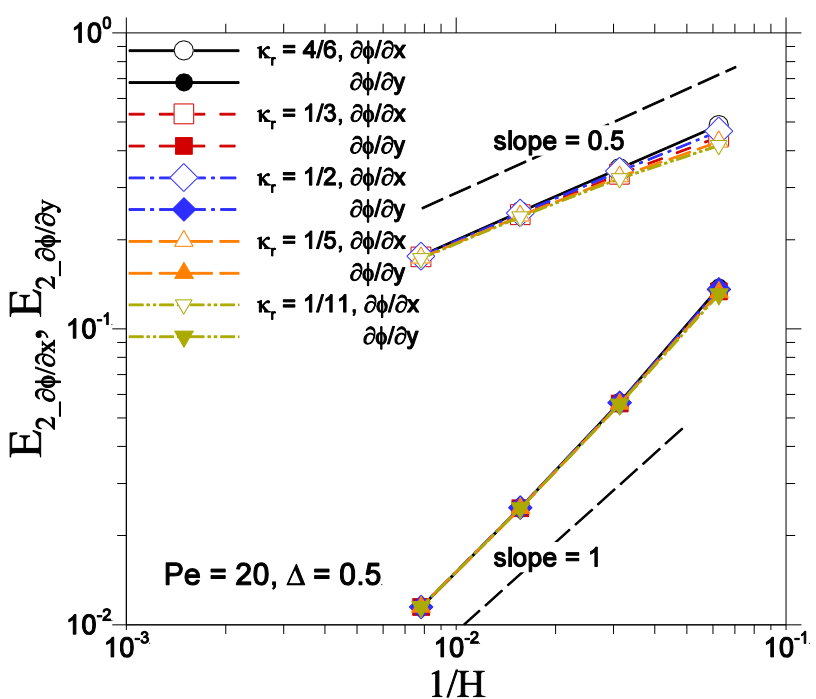

(b)

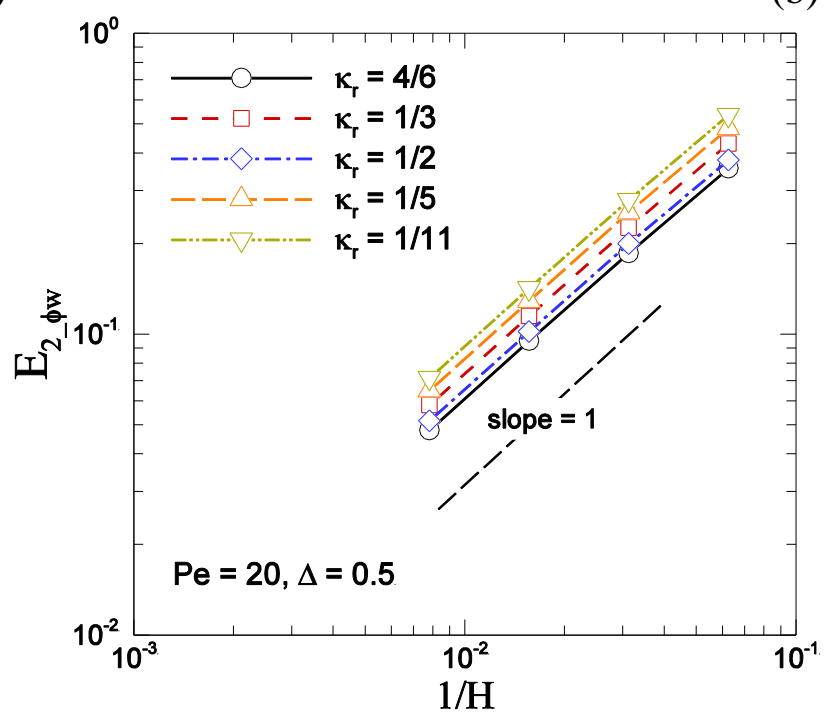

(c)

Fig. 17. Relative $L_{2}$-norm errors, (a) $E_{2}$, (b) $E_{2 \_} \partial \phi / \partial x$ and $E_{2 \_} \partial \phi / \partial y$, and (c) $E_{2 \_\phi w}$ versus the grid resolution, $1 / H$, for the steady convection-diffusion in a $2 \mathrm{D}$ channel with Neumann conditions at $P e=20$ and $\Delta=0.5$ by using various flux splitting weights $\kappa_{r}$ and $\kappa_{d}\left(\kappa_{r}+2 \kappa_{d}=1\right)$. The D2Q9-I model is used in the simulations. 


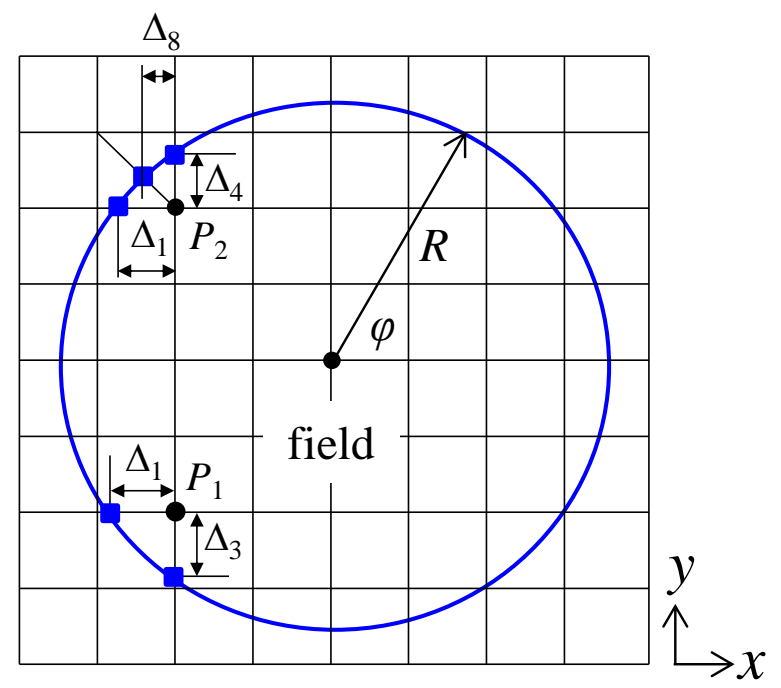

Fig. 18. Schematic layout of the lattice around a circle. The intersection $\Delta$ values in all lattice directions, $\mathbf{e}_{\bar{\alpha}}$, are calculated for each lattice node adjacent to the curved boundary, e.g., the representative nodes $P_{1}$ in $\mathrm{D} 2 \mathrm{Q} 5$ and $P_{2}$ in D2Q9.

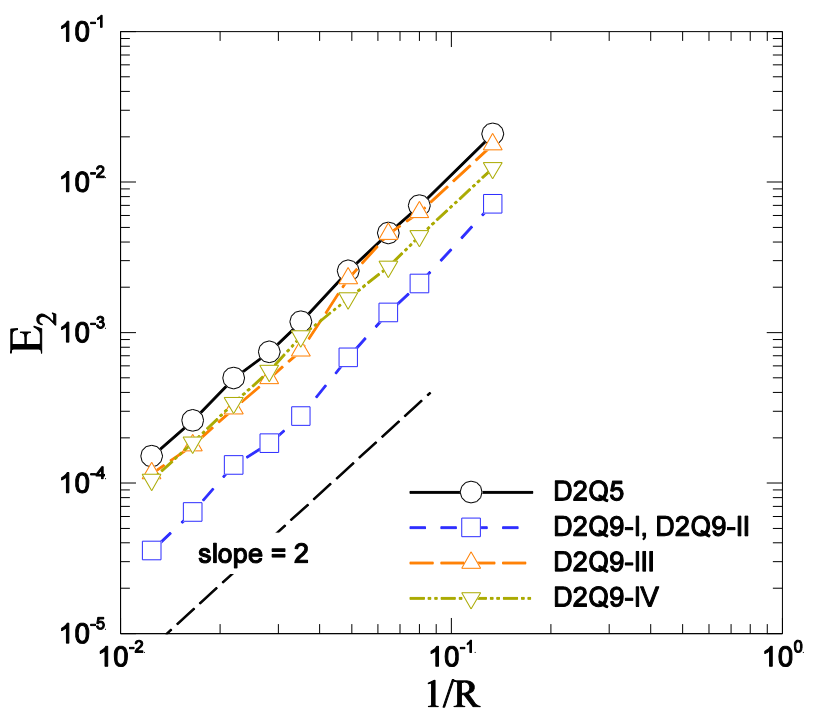

(a)

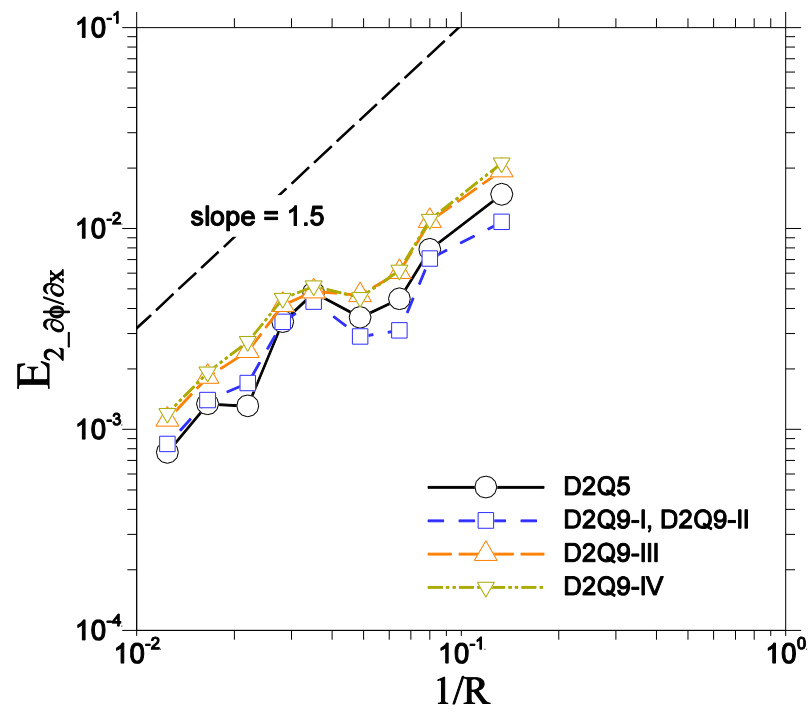

(b)

Fig. 19. Relative $L_{2}$-norm errors, (a) $E_{2}$, and (b) $E_{2} \partial \phi / \partial x$ versus the grid resolution, $1 / R$, for the steady diffusion in a circle with a Dirichlet condition. 


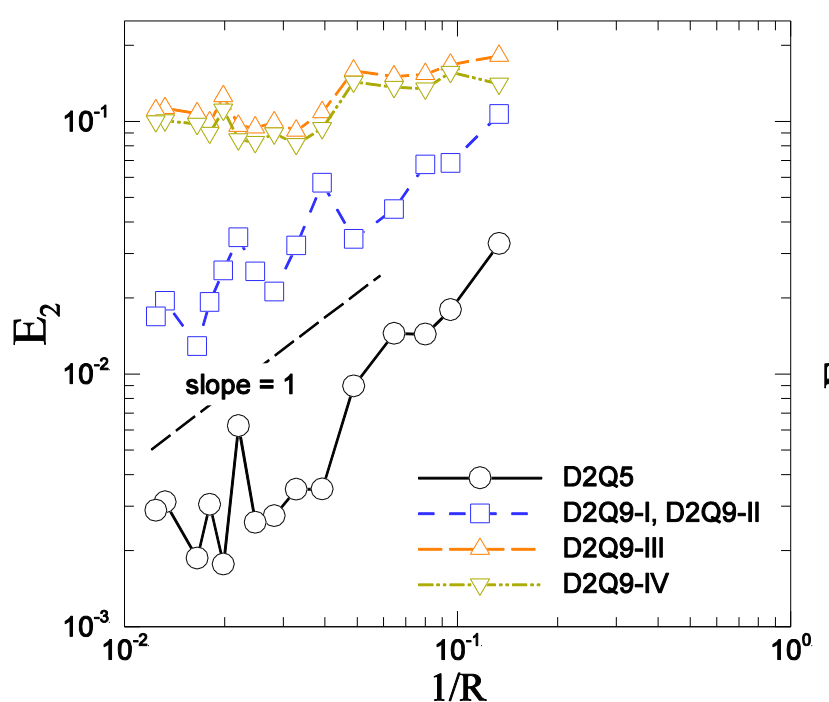

(a)

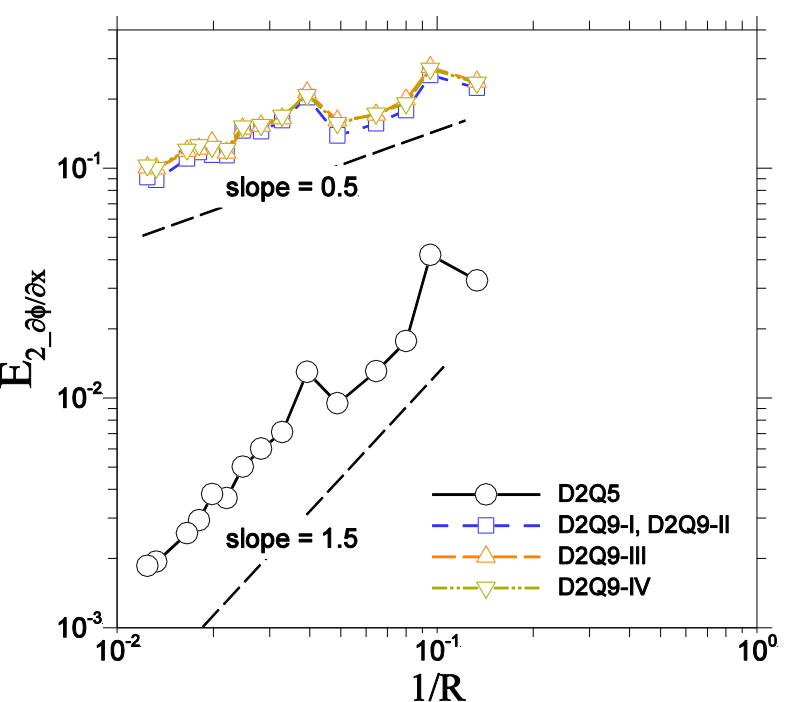

(b)

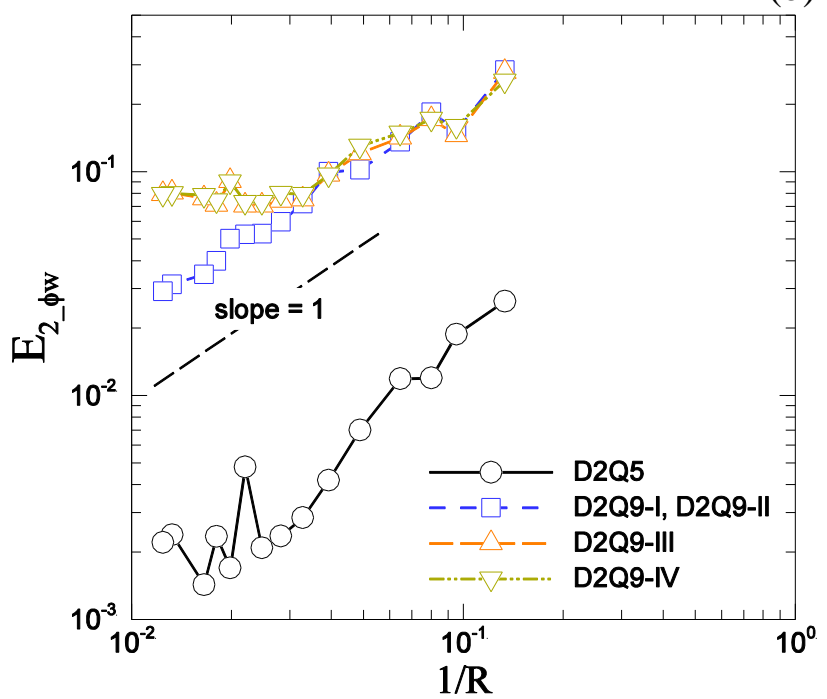

(c)

Fig. 20. Relative $L_{2}$-norm errors, (a) $E_{2}$, (b) $E_{2 \_} \partial \phi / \partial x$, and (c) $E_{2 \_\phi w}$ versus the grid resolution, $1 / R$, for the steady diffusion in a circle with a Neumann condition. 


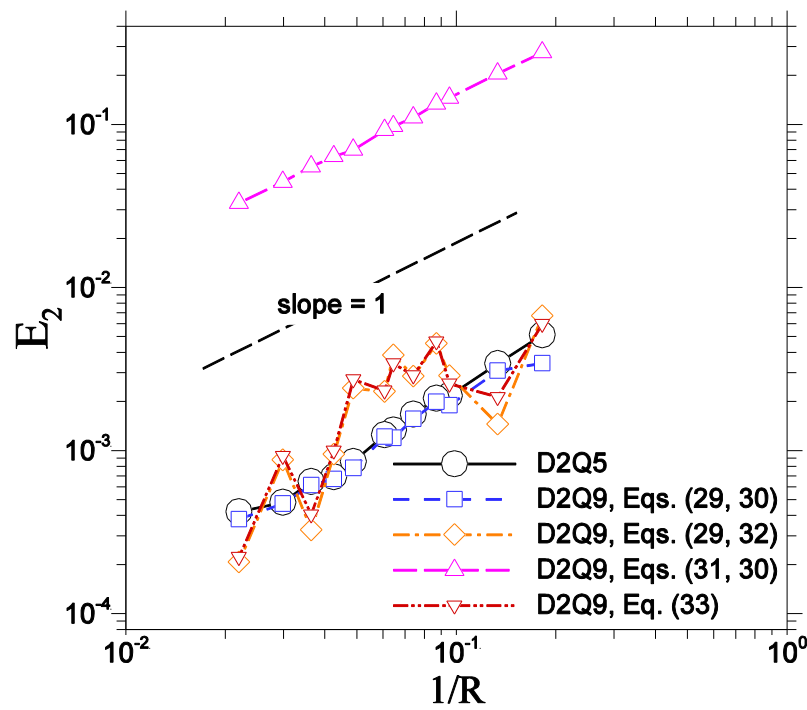

Fig. 21. The error $E_{2}$ defined in Eq. (50) for the boundary flow rate versus the grid resolution, $1 / R$, for the transient diffusion in a circle with a Dirichlet condition.

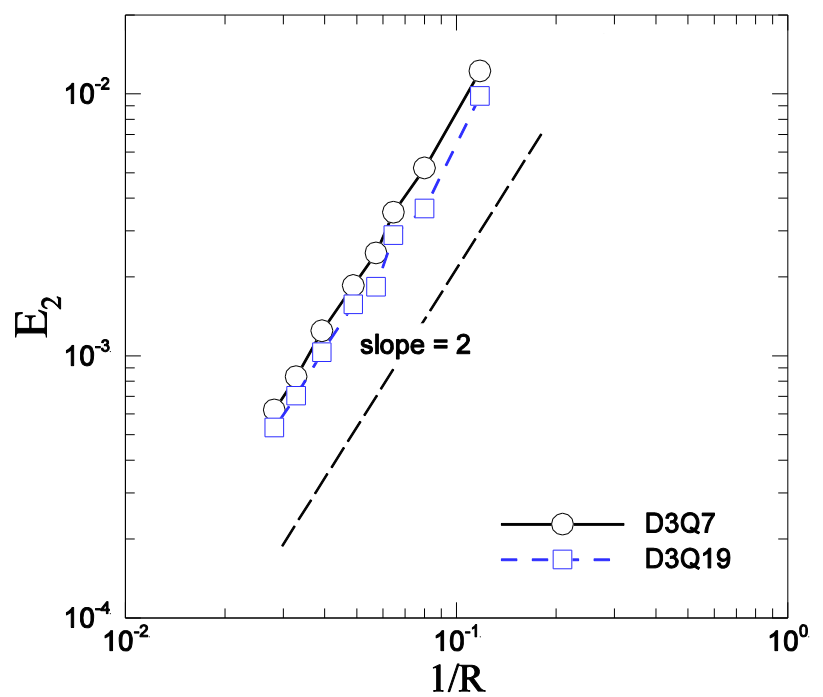

(a)

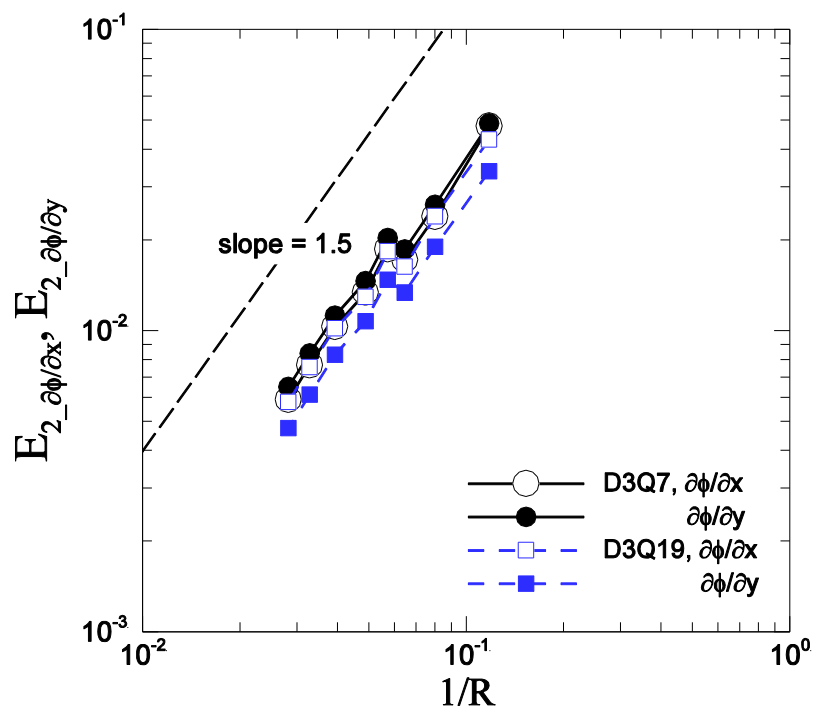

(b)

Fig. 22. Relative $L_{2}$-norm errors, (a) $E_{2}$, and (b) $E_{2 \_} \partial \phi / \partial x$ and $E_{2 \_} \partial \phi / \partial y$ versus the grid resolution, $1 / R$, for the steady diffusion in a sphere with a Dirichlet condition. 